\title{
A PREDICTIVE MODEL OF ADOLESCENT PERSISTENCE IN COUNSELING
}

\author{
Jessica M. Holm
}

Dissertation Prepared for the Degree of

DOCTOR OF PHILOSOPHY

UNIVERSITY OF NORTH TEXAS

May 2015

APPROVED:

Casey Barrio Minton, Major Professor Elizabeth Prosek, Committee Member Delini Fernando, Committee Member Amanda Giordano, Committee Member Jan Holden, Chair of the Department of Counseling and Higher Education Jerry Thomas, Dean of the College of Education Costas Tsatsoulis, Interim Dean of Toulouse Graduate School 
Holm, Jessica M. A Predictive Model of Adolescent Persistence in Counseling. Doctor of Philosophy (Counseling), May 2015, 138 pp., 4 tables, references, 81 titles.

Adolescence is a developmental period characterized by profound social and emotional changes. Counseling can serve as a protective factor for decreasing the long-term negative emotional effects. Despite this fact, counselors continue to struggle with high rates of attrition among adolescent clients. When examining trends of client persistence in counseling across the lifespan, researchers found a relationship between the presence of internalizing and externalizing behaviors. Additionally, they found that high levels of familial stress predicted premature termination from counseling.

The purpose of the current study was to create a predictive model of adolescent persistence in counseling. I examined both personal and environmental characteristics of adolescents who sought counseling services $(N=72)$ from an on-campus university counseling clinic that serves as a training facility for master's and doctoral students at an accredited counselor education program in the southwestern United States. Participants were predominantly White $(67.6 \%, n=50)$, with a mean age of $14.23(S D=1.65)$. Nearly $60 \%(n=$ 44) of the clients were female, and $37.8 \%$ were male $(n=28)$. Beyond descriptive variables, eight predictor variables were examined: adolescent involvement in their intake, time spent on the wait list, four domains of parental stress, and parent perceptions of adolescent internalizing and externalizing behaviors. A multiple linear regression was conducted to understand how much of the variance in the number of counseling sessions attended by adolescent clients was explained by the predictor variables. The regression analysis was statistically significant $(p=$ $.008)$ and accounted for $29.1 \%\left(R^{2}\right.$ adj $\left.=.192\right)$ of the variance in sessions attended. Of the variables examined, externalizing behaviors (42.82\%) accounted for the most variance in 
internalizing behaviors (12.96\%), and parent-focused stress (10.30\%). An examination of the two strongest predictors in correlation to the number of sessions attended revealed a negative relationship with externalizing behaviors and a positive relationship with involvement in the intake, suggesting that adolescents who present with externalizing behaviors and who are not involved in their intake are likely to attend fewer counseling sessions.

To understand the differences among adolescents who prematurely terminate from counseling and those who persist in counseling, I conducted a series of univariate and multivariate analyses. Comparing the means of the predictor variables revealed no significant differences between any of the predictor variables examined, although internalizing and externalizing behavior scores fell within the borderline clinical range for adolescents who persisted in counseling. Finally, a predictive discriminant analysis to assess to what degree identified variables predicted premature termination from or persistence in counseling yielded a $75 \%$ hit rate. Given this knowledge, counselors may be able to pro-actively identify adolescents who are at risk of prematurely terminating from counseling and implement retention strategies. 
Copyright 2015

By

Jessica M. Holm 


\section{ACKNOWLEDGEMENTS}

Firstly, I would like to thank my incredibly supportive committee for their dedication to my personal and professional growth. Dr. Casey Barrio Minton, you encouraged me to go beyond my comfort zone and provided me with professional opportunities that I will carry with me as a counselor educator. You are truly an inspiration. Dr. Elizabeth Prosek, you have been an absolute lifesaver! The ability for me to be unconditionally genuine, regardless of the circumstance, allowed me to not only survive this crazy process, but to flourish. You are a part of every accomplishment I will take from this journey. Dr. Amanda Giordano, your research mentorship has been invaluable. I thank you for demonstrating how I can transfer my passion for research to my students. Dr. Delini Fernando, thank you for all of your support, your kind smile always brought me back to the current moment. I am thankful for all of the UNT Counseling Faculty. Through your teaching, supervision, and mentorship, each of you contributed to the development of my identity as a counselor educator. Dr. Dee Ray, thank you for always encouraging me to reflect on I could push myself to better serve my clients. I also thank you for allowing me to utilize the CFRC data for my dissertation. To my cohort members and peers who pained along this journey with me- Thank You! Each of you have such bright futures and I cannot wait to see what life has in store for you!

Without the support of my family and friends, I would have never believed that I was capable of achieving my dreams. There is absolutely no question that I could have done this without my husband, Dustin Holm. You supported me through all the late nights, grumpiness, and forgotten household duties. You made countless sacrifices to ensure that I was able to pursue my dreams and I am eternally grateful. I' m beyond fortunate to have you and I cannot wait to see where life takes us. 
ACKNOWLEDGEMENTS ........................................................................................... ii

A PREDICTIVE MODEL OF ADOLESCENT PERSISTENCE IN COUNSELING ................. 1

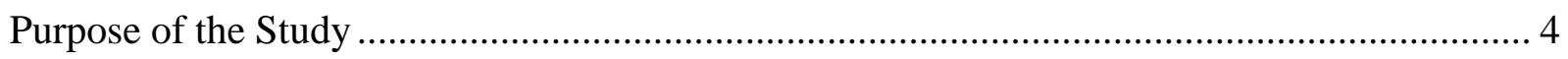

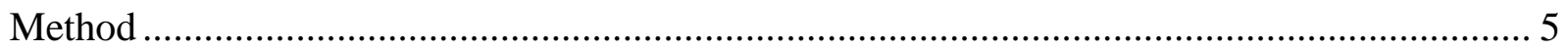

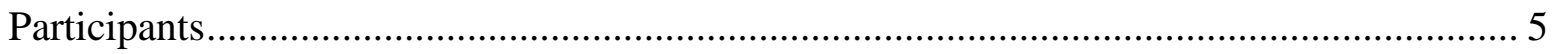

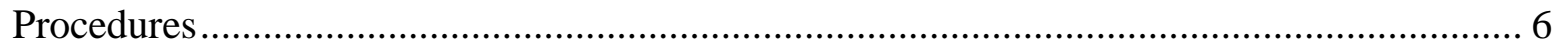

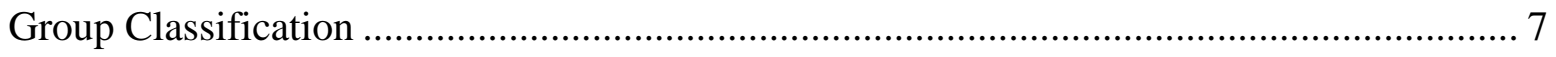

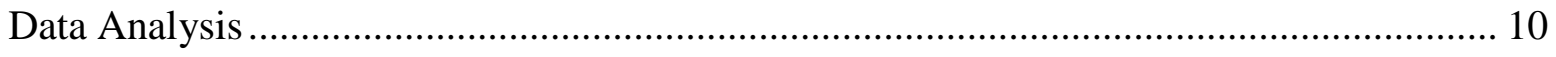

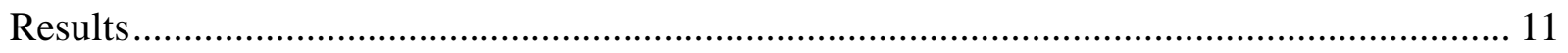

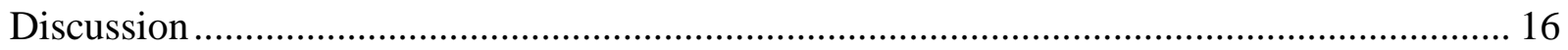

Implications for Clinical Practice ...................................................................... 21

Limitations and Suggestions for Future Research ....................................................... 22

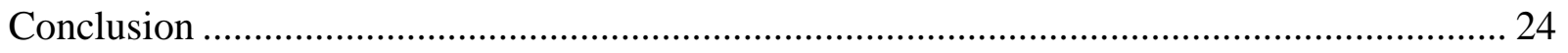

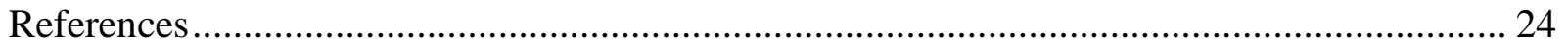

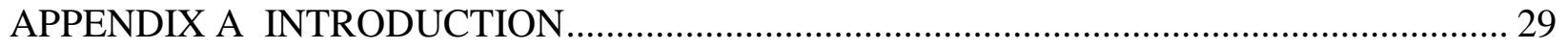

APPENDIX B EXTENDED LITERATURE VIEW ….................................................... 36

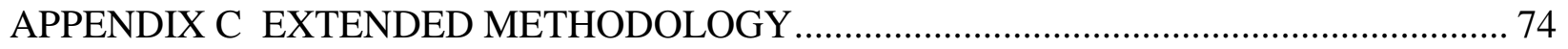

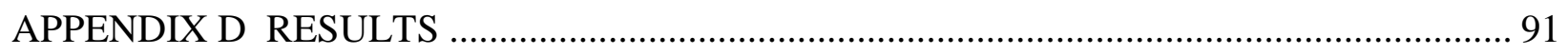

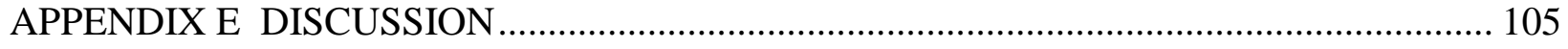


APPENDIX F SUPPLEMENTAL MATERIALS......................................................... 119

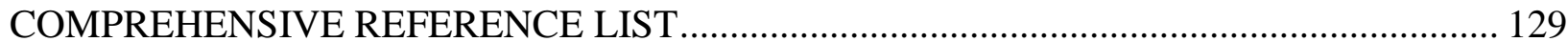




\section{A PREDICTIVE MODEL OF ADOLESCENT PERSISTENCE IN COUNSELING}

Adolescence is a critical time of development characterized by significant changes that occur as individuals transition from childhood to adulthood (Ausubel, 2002). Changes in adolescents' biological, cognitive, and emotional development can lead to significant mental health concerns and an increase in high-risk behaviors. One in eight adolescents experience mental illness that significantly impairs daily functioning, and nearly half of all mental illness experienced by adults begins during adolescence (Belfer, 2008). Nationwide, nearly $40 \%$ of adolescents met criteria for anxiety disorders, and $20 \%$ met criteria for conduct disorders (Merikangas, Nakamura, \& Kessler, 2010). High-risk behaviors such as substance use and reckless driving contribute to leading causes of death among this age group (World Health Organization, 2014). To address these concerns, adolescents may benefit from attending counseling.

Although counseling may serve as a protective factor for prolonged emotional concerns, only one in five youth struggling with mental illness received appropriate treatment for mental health concerns (National Alliance on Mental Illness, 2013). Counseling is effective at decreasing mental health concerns and maladaptive behaviors across the lifespan (Enea \& Dafinoiu, 2009; Kadera, Lambert, \& Andrews, 1996), with therapeutic change occurring after only eight sessions (Lambert, 2013). Despite the benefits obtained by persisting in counseling, nearly half of all clients terminate prematurely (Wierzbicki \& Pekarik, 1993). Clients who terminate from counseling prematurely are less likely to benefit from treatment than clients who persist in counseling (Prinz \& Miller, 1994).

Across the lifespan, researchers have examined personal variables (e.g., demographic characteristics, presenting problems) and environmental variables (e.g., familial characteristics, 
the counseling process) to understand client persistence or premature termination from counseling. There is limited knowledge of the specific characteristics that are predictive of adolescents either persisting or prematurely terminating from counseling. What is known is that adolescents are more likely to remain engaged in counseling if they self-identify internalizing behaviors (i.e., feelings of anxiety or depression) or if they seek treatment for themselves (Baruch, Vroura, \& Fearon, 2009; Pagnin, de Queiroz, \& Saggese, 2004; Sunseri, 2003). In contrast, adolescents who engage in externalizing behaviors (i.e., delinquency or aggression), or are referred to counseling by an external source (e.g., teachers, judicial system) are more likely to terminate counseling prematurely (Baruch et al., 2009; Pagin et al., 2004; Sunseri, 2003). Despite developmental differences between childhood and adolescence, counseling researchers often examine youth simultaneously. When doing so, demographic variables such as age, gender, and ethnicity were not found to be predictive for treatment persistence (Dierker Nergiso, Wiseman, \& Hoff, 2001). In comparison to findings specific to adolescence, researchers found conflicting results regarding the relationship between externalizing behaviors and premature termination from counseling in children (Dierker et al., 2001; Kazdin, Holland, \& Crowley, 1997). Parental influences also played a significant role in children and adolescents’ persistence in counseling. Familial characteristics were also examined; adolescents from families whom were headed by a single parent, reported low socioeconomic status (SES), or were from a non-dominant ethnic group were less likely to persist in counseling (Kazdin et al., 1997). Overall, adolescents whose parents engaged in unhealthy parenting styles, did not have strong positive beliefs about the treatment process, and had high levels of stress were more likely to drop out from counseling prematurely (Kazdin et al., 1997; Venable \& Thompson, 1998). 
An examination of treatment persistence among children revealed several other findings. Researchers examining attrition in child counseling found that increased severity of symptoms at the onset of counseling was associated with premature termination (Kazdin \& Mazurick, 1994; Tsai \& Ray, 2011). This increase in premature termination may be associated with the impact on the family system, along with the extended length of treatment needed to experience meaningful change (Kazdin \& Mazurick, 1994; Tsai \& Ray, 2011). Congruent with the research on children and adolescents, parental stress, adverse parenting styles, and parents' beliefs about counseling and their involvement in the process played a significant role in the persistence of their children in counseling (Attride-Stirling, Davis, Farrell, Groark, \& Day, 2004; Kazdin \& Mazurick, 1994; Kazdin, Stolar \& Maciano, 1995; Prinz \& Miller, 1994; Tsai \& Ray, 2011). In all, it is evident that environmental factors impact children’s' persistence in counseling.

An examination of research on attrition across the lifespan also included research on adult clients. Researchers examined persistence in adult clients in two dominant treatment settings: university-based clinics and specialized inpatient programs. Across studies, adults who reported low SES, had lower levels of education, and identified from non-dominant ethnic groups were more likely to terminate from services prematurely (Reis \& Brown, 1999; Wiezbicki \& Pekarik 1993). Further, increased time spent on the waitlist to receive services was associated with premature termination (Long et al., 1992). Clients' levels of motivation and selfefficacy were associated with persistence in counseling (Chang \& Saunders, 2002). This finding suggests that identifying internal motivators may serve as a protective factor against premature termination.

Across the lifespan, it is evident that clients' motivation to seek treatment and familial support are important factors in their decision to persist in counseling. Although adolescents' 
internalizing behaviors were predictive of persistence in counseling and externalizing behaviors were predictive of premature termination from counseling (Baruch et al., 2009; Pagnin et al., 2004; Sunseri, 2003), these findings were inconsistent throughout the lifespan (Dierker et al., 2001; Kazdin, Holland, \& Crowley, 1997). Additionally, households characterized by high levels of parental stress were associated with high rates of attrition for children and adolescents (Attride-Stirling et al., 2004; Kazdin \& Mazurick, 1994; Kazdin et al., 1995; Prinz \& Miller, 1994; Tsai \& Ray, 2011). Although research on the lifespan provides additional information on understanding factors that are predictive of client persistence, adolescent persistence remains understudied.

\section{Purpose of the Study}

Client persistence in counseling can lead to a decrease in pervasive mental health problems and increase overall levels of functioning (Lambert, 2013). If counselors can better understand characteristics that are predictive of adolescent persistence in counseling, counselors will be able to better identify clients who are at risk for premature termination. By gaining this knowledge, counselors may be able to develop retention strategies to increase the likelihood of clients persisting in counseling, thus decreasing the likelihood that distress experienced during adolescents manifest in lifelong mental health concerns (Harris, 1998). The purpose of the current study was to develop a predictive model of adolescent persistence in counseling. This is achieved through an examination of the following research questions:

1. How much of the variance in the number of counseling sessions attended by adolescent clients is explained by adolescent involvement in intake, time spent on wait list, levels of parental stress, and parent perceptions of adolescent internalizing and externalizing behaviors? 
2. What is the demographic and clinical profile of adolescent clients who prematurely terminated from counseling compared to those who persisted in counseling?

3. To what degree can gender, age, whether an adolescent is involved in intake, time spent on wait list, levels of parental stress, and parent perceptions of adolescent internalizing and externalizing behaviors predict whether an adolescent will terminate prematurely or persist in counseling?

\section{Method}

\section{Participants}

Archival data were acquired from clinical files $(N=74)$ of adolescent clients who sought services at a university-based community-counseling clinic between 2007 and 2014. The clinic served as a training facility at an accredited counselor education program in the southwestern United States and served approximately 150 clients per week. An array of treatment modalities were available including play therapy; activity therapy; and individual, group, couple, and family counseling. Services were provided on a sliding scale fee structure. The majority of the counselors were doctoral students who served clients during the second year and beyond in their doctoral program. Several master's level counselors also completed their internship at the clinic. All counselors engaged in weekly supervision.

Selection of participants was based on several inclusion criteria. Firstly, clients were between the ages of 12 and 18 at the time of intake. The participants must have also been living with caretakers and have completed an intake session requesting individual counseling services. Additionally, the required assessments, the Child and Adolescent Background Information Form (CABIF), an Intake Summary Report, a Child Behavior Checklist (CBCL; Achenbach \& 
Rescorla, 2001), and a Stress Index for Parents of Adolescents (SIPA; Sheras, Abidin, \& Konold., 1998), must have been completed within the file.

Of the adolescent files examined $(N=74), 89.2 \%(n=66)$ were closed-client files and $10.2 \%(n=8)$ were clients currently receiving services. The sample was $61.1 \%(n=44)$ female and 38.9\% ( $n=28)$ male, with two participants not reporting their gender. The mean age of the sample was $14.23(S D=1.65)$ and the mean grade in school was $8.44(S D=2.30)$. Participants identified as White (67.6\%, $n=50)$, Hispanic/Latino(a) (14.9\%, $n=11)$, Bi-racial (10.8\%, $n=$ 8), African American (4.1\%, $n=3)$, and Asian $(2.7 \%, n=2)$. An examination of family household income revealed annual household income as follows: less than $\$ 15,000$ (20.7\%, $n=$ 12), $\$ 15,001-\$ 20,000(12.1 \%, n=7)$, $\$ 20,001-\$ 30,000(15.5 \%, n=9)$ ), $\$ 30,001-\$ 40,000(12.1 \%$, $n=7)$, and $\$ 40,001+(39.7 \%, n=23)$. Sixteen (21.6\%) participants did not report household income.

Procedures

After obtaining permission from the clinical director to create a database of the adolescent client files for research purposes, I obtained approval from the University Institutional Review Board. Due to utilization of archival data, clinic procedures were already established. Parents or guardians interested in services for their adolescents attended an intake session at the clinic. Upon arrival, they completed paperwork that included a CABIF, Intake Summary Report, CBCL, and SIPA. They then completed an intake session during which a counselor obtained informed consent regarding the nature of services at the training clinic and use of data for education and research purposes. Upon completing the clinical intake, clients were put on a waiting list until being scheduled by a counselor for weekly 50-minute sessions. 


\section{Group Classification}

In the current study, persistence was defined as attending at least eight counseling sessions. In a meta-analysis, Lambert (2013) found most clients show clinically significant improvement after seven sessions, and resolution of presenting problems occurs after eight sessions. Thus, it was determined that clients who attended at least eight sessions met the criteria for persisting in counseling. Conversely, premature termination was defined as a not completing at least two counseling sessions thus limiting the external effects of the therapeutic experience. Instrumentation

Child and Adolescent Background Information Form (CABIF). The clinical director developed the CABIF to gather biopsychosocial information on clients. The CABIF was also utilized to collect information regarding the client's presenting concerns and familial stressors. The current study examined the following demographic variables: age, gender, grade, race/ethnicity, SES.

Intake Summary Report. The clinical director developed the intake summary report to document details of the intake session. For the current study, I examined the form to determine the number of days between intake and first scheduled counseling session, which represented the amount of days spent on waitlist. The document was also examined to determine whether the adolescent was involved in the intake session.

Child Behavior Checklist 6-18 (CBCL; Achenbach \& Rescorla, 2001). The CBCL is an instrument that measures adolescents' behavioral and emotional problems. The CBCL is completed by parents or guardians and designed for youth aged 6-18. Parents are asked to indicate the occurrence of behaviors within the past six months. Results are reported according to three behavioral domains: internalizing, externalizing, and total behavior. According to 
Achenbach and Rescorla (2001), externalizing behaviors are defined as the outward expression of internal distress (e.g., aggression, delinquency). In contrast, internalizing behaviors are defined as the inward expression of internal distress (e.g., depression, anxiety). Parents respond to 13 questions regarding their child's social interactions, school experience, and activity involvement to capture a holistic view of the child from the respondent's perspective. The parents then respond to 113 Likert-scale questions to capture their children's behavior during the previous six months. Scaling of questions ranges from: " 0 = not true", " 1 = somewhat or sometimes true", to " 2 = very true or often true." Scores are reported based on percentiles and normalized $T$-scores to indicate a child's functioning compared to other children of the same sex and age. Researchers are able to examine normative data to determine if children's scores fall within the clinical or non-clinical range.

Achenbach and Rescorla (2001) reported the use of the CBCL across several studies during the past 20 years, establishing strong validity for the assessment. Content and criterionrelated validity were established, finding that across all items, the CBCL discriminated between demographically comparable referred and non-referred children. Although the scoring system for the CBCL did not allow us to compute internal consistency reliabilities for the CBCL in the current sample, the CBCL was found to have high reliability in test samples, with a Cronbach's alpha of .90 to .97 for the three subscales and high inter-interviewer reliability (.93 to .96). Testretest reliability was also found to be strong, ranging from .95 to 1.00 .

To capture parent perceptions of adolescent mental health concerns, I examined $T$-scores from internalizing and externalizing subscales. Sample questions for internalizing behaviors include: "Cries a lot," "Rather be alone”; and externalizing behaviors: "Harms self,” "Cruel to 
animals.” Scores on the CBCL are categorized based on a continuum that ranges from: nonclinical (<65), to borderline clinical (65-70), and clinical (>70; Achenbach \& Rescorla, 2001). High $T$-scores on the scales indicate clinically significant deviance from the behavior to be expected for that gender and age range.

Stress Index for Parents of Adolescents (SIPA; Sheras et al., 1998). The SIPA is a 112item instrument that measures stress related to a caretaker's interactions with an identified adolescent between the ages of 11 and 19. The SIPA is an adaptation of the Parenting Stress Index and is designed to capture changes in parents' process as their children move into adolescence (Abidin, 1993). The assessment examines stress on a 5-point Likert scale ranging from: "1= Strongly Agree” to " 5 = Strongly Disagree." Stress is reported across four domains: parent-domain, adolescent-domain, adolescent-parent relationship, and life stressors.

Due to the utilization of archival data, internal consistency of the SIPA with the current sample was not accessible. Despite this, Sheras et al. (1998) established a high degree of reliability for the SIPA with a moderate to high degree of internal reliability (alpha coefficients = .81-.97) and test-retest reliability (.74-.93; Sheras et al., 1998). Sheras et al. (1998) also established convergent, discriminant, and construct validity. The high validity indicates the SIPA can effectively assess parents struggling with stress related to parenting their adolescents as well as identifying an adolescent's diagnosis based on Diagnostic and Statistical Manual Fourth Edition Text Revision (DSM-IV; American Psychological Association [APA], 2000) criteria.

To understand levels of parental stress, all four domains were examined. The parentdomain contains 36 questions and "measures the level of stress experienced by a parent as a function of the effect on parenting on other life roles, the relationship with a spouse or partner, 
social isolation, and parenting competence" (p. 6). The adolescent-domain "measures the level of stress experienced by a parent as a function of the characteristics of his or her adolescent” (p. 6). The adolescent-domain contains 40 likert-scale questions and assesses adolescent attributes including: moodiness/emotional liability, social isolation/withdrawal, delinquency/antisocial, and failure to achieve or persevere. The adolescent-parent relationship domain "measures the perceived quality of the relationship that the parent has with the adolescent, such as the degree of communication and affection between them” (p. 6). The adolescent-parent relationship domain is a 16 question scale that assesses the quality of the relationship as experienced by the caretaker through questions measuring the level of communication, self-perceived confidence in the parental role, and quality of their interactions. The life stressors domain provides an opportunity to "indicate the number of stressful evens that the parent has experienced in the past year” (p. 6). This may include loss of a job, death of a loved one, or illness. Combining scores across all four subscales yields a cumulative score, total stress. Due to the cumulative nature of the total stress scale, it was not examined in the current study.

Data Analysis

To address the Research Question 1, I conducted a linear multiple regression to understand how much of the variance in the number of counseling sessions attended was explained by the predictor variables. Due to the dependent variable (number of sessions attended) being continuous in nature, only closed client files $(n=66)$ were included in this analysis. A priori analysis using G*Power 3.1 (Faul, Erdfelder, Buchner \& Lang, 2009) indicated a sample size of 43 was needed to detect a moderate effect size, $\left(f^{2}=.15\right)$, power $=.80, \alpha=.05$, for the eight predictor variables examined. Thus, the available sample exceeded the required sample size. I utilized standard entry to simultaneously examine the predictor variables (Field, 
2009). I also conducted a series of preliminary analyses to confirm that the data met the assumptions of linearity, normality, and homoscedasticity.

To address Research Question 2, I ran descriptive statistics to examine differences among demographic variables for adolescents who prematurely terminated from counseling and those who persisted in counseling $(N=59)$. Subsequently, a predictive discriminant analysis (PDA) was conducted to determine to what degree the variables could predict whether an adolescent terminated prematurely or persisted in counseling (Sherry, 2006). The grouping variable was identified as adolescents' completion of treatment: those who prematurely terminated from counseling and those who persisted in counseling. Based on a review of the literature, predictor variables were those that the researcher deemed important to the exploration of group difference and included: gender, age, time spent on the waitlist, adolescent involvement in the intake, internalizing and externalizing behaviors, and four domains of parental stress. According to guidelines established by Poulsen and French (2008), there should be four to five times the number of total cases for each predictor variable. Thus, the cases analyzed $(n=59)$ exceeded the number of cases needed to examine the 10 predictors $(n=50)$. For the PDA analysis, I also utilized standard entry to simultaneously examine the predictor variables (Field, 2009). Preliminary analyses revealed that the data met the assumptions of linearity, normality, and homogeneity of variance.

\section{Results}

\section{Research Question 1}

A linear multiple linear regression was conducted to explore the degree to which adolescent involvement in intake, time spent on wait list, levels of parental stress, and parent perceptions of adolescent internalizing and externalizing behaviors explained variance in the 
number of counseling sessions attended by adolescent clients. To meet the needs of the current research question, only closed-client files were included in this analysis $(n=64)$. As indicated in Table 1 , the model was statistically significant $(F(8,57)=2.930, p=.008)$ and accounted for $29.1 \%\left(R^{2}=.291, R^{2}\right.$ adj $\left.=.192\right)$ of the variance in number of sessions attended. According to Cohen (1992) this model had a large effect size.

Table 2 displays standardized regression coefficients $(\beta)$, squared structure coefficients $\left(r_{\mathrm{s}}{ }^{2}\right)$, and bivariate correlations for each predictor variable. Although the standardized regression coefficient for externalizing behaviors was not statistically significant $(\beta=-.205, t=-.954, p=$ .344) an examination of the squared structure coefficients revealed the presence of suppressor effects within the model (Kraha, Turner, Nimon, Zientek, \& Hensen, 2012). Externalizing behaviors accounted for $43.82 \%$ of the variance explained in number of sessions attended. Whether the adolescent was involved in the intake (29.16\%), internalizing behaviors (12.96\%), and parent-focused stress (10.30\%) also contributed to variance explained in number of sessions attended. Remaining variables contributed minimally to the model: life stressors (3.9\%), adolescent-parent relationship (0.29\%), adolescent-focused stress ( $0.22 \%)$, and time spent on the waitlist (0.12\%). An examination of correlations revealed both positive and negative relationships between variables and the number of sessions attended. Whether the adolescent was involved in the intake, internalizing behaviors, adolescent-parent relationship, and life stressors were positively correlated with number of sessions attended. Conversely, number of days spent on the waitlist, externalizing behaviors, adolescent-focused stress, and parent-focused stress were negatively correlated with the number of sessions attended by the adolescent. 
Table 1

Regression Summary Table

\begin{tabular}{lccccccc}
\hline Model & SOS & $d f$ & Mean Square & F & P & $\mathrm{R}^{2}$ & $\mathrm{R}^{2}{ }_{\text {adj }}$ \\
\hline Regression & 2986.557 & 8 & 373.320 & 2.930 & .008 & .291 & .192 \\
Residual & 7262.973 & 57 & 127.421 & & & & \\
Total & 10249.530 & 65 & & & & & \\
\hline
\end{tabular}

Table 2

Beta Weights and Structure Coefficients for the Regression Model ( $n=66)$

\begin{tabular}{|c|c|c|c|c|c|}
\hline Variables & $\boldsymbol{\beta}$ & $t$ & $p$ & $\mathbf{r s}^{2}$ & $\mathbf{r}$ \\
\hline Involvement in Intake & -.092 & -.791 & .432 & .292 & .018 \\
\hline Time on Waitlist & -.190 & -1.515 & .135 & .001 & -.195 \\
\hline Internalizing Behaviors & -.468 & 3.422 & .001 & .129 & .357 \\
\hline Externalizing Behaviors & -.205 & -.954 & .344 & .438 & -.025 \\
\hline Adolescent-Focused Stress & -.286 & -1.569 & .122 & .002 & -.173 \\
\hline Parent-Focused Stress & -.021 & -1.65 & .869 & .103 & -.029 \\
\hline Adolescent-Parent & .264 & 1.903 & .062 & .003 & .106 \\
\hline Relationship Stress & & & & & \\
\hline Life Stressors & .195 & 1.456 & .151 & .039 & .027 \\
\hline
\end{tabular}

In order to understand the profile of adolescents who prematurely terminated from counseling compared adolescents who persisted in counseling groups on each of the demographic and clinical variables, descriptive statistics are presented in Tables 3 and 4.

To meet the needs of the current research questions, only clients who prematurely terminated $(59 \%, n=35)$ or persisted $(41 \%, n=24)$ in counseling were included in the analysis. Analysis of the data revealed that only a small portion $(n=13)$ of clients attended between two and seven sessions and therefore were not included as a distinct group in the analyses. 
Table 3

Demographic Descriptive Comparisons

\begin{tabular}{|c|c|c|c|c|}
\hline \multirow[b]{2}{*}{ Demographic Variable } & \multicolumn{2}{|c|}{$\begin{array}{l}\text { Prematurely Terminates } \\
\qquad(n=35)\end{array}$} & \multicolumn{2}{|c|}{$\begin{array}{l}\text { Persists } \\
(n=24)\end{array}$} \\
\hline & $M$ & SD & $M$ & $S D$ \\
\hline Age & 14.69 & 1.49 & 13.50 & 1.64 \\
\hline \multirow[t]{2}{*}{ Grade } & 8.85 & 2.31 & 8.09 & 1.81 \\
\hline & $N$ & $\%$ & $N$ & $\%$ \\
\hline \multicolumn{5}{|l|}{ Gender } \\
\hline Female & 15 & 42.9 & 18 & 75.0 \\
\hline Male & 20 & 57.1 & 6 & 25.0 \\
\hline \multicolumn{5}{|l|}{ Race/ethnicity } \\
\hline Asian & 0 & 0.0 & 1 & 4.2 \\
\hline African American & 2 & 5.9 & 0 & 0.0 \\
\hline Bi-racial & 1 & 2.9 & 5 & 20.8 \\
\hline Hispanic Latino(a)/ & 4 & 11.4 & 4 & 16.7 \\
\hline White & 28 & 80.0 & 15 & 58.3 \\
\hline \multicolumn{5}{|l|}{ SES } \\
\hline$<\$ 15,000$ & 8 & 22.9 & 2 & 8.3 \\
\hline$\$ 15,001-\$ 20,000$ & 4 & 11.4 & 2 & 8.3 \\
\hline$\$ 20,001-\$ 30,000$ & 6 & 17.1 & 3 & 12.5 \\
\hline$\$ 30,001-\$ 40,000$ & 3 & 8.6 & 3 & 12.5 \\
\hline$>\$ 40,001$ & 11 & 31.4 & 7 & 29.2 \\
\hline
\end{tabular}


Table 4

Predictor Variable Descriptive Statistics

\begin{tabular}{|c|c|c|c|c|}
\hline Predictors & $\begin{array}{l}\text { Prematurely } \\
\text { Terminates } \\
(n=35)\end{array}$ & & $\begin{array}{l}\text { Persists } \\
(n=24)\end{array}$ & \\
\hline \multirow{2}{*}{$\begin{array}{l}\text { Adolescent involvement in } \\
\text { intake }\end{array}$} & No $=71.4 \%$ & Yes $=28.6 \%$ & No $=70.8 \%$ & Yes $=29.2 \%$ \\
\hline & Mean & $S D$ & Mean & SD \\
\hline Time on wait list & 71.86 & 61.25 & 57.00 & 52.16 \\
\hline Internalizing Behaviors & 60.89 & 9.94 & 65.67* & 11.12 \\
\hline Externalizing Behaviors & 60.17 & 10.04 & $66.64 *$ & 12.17 \\
\hline $\begin{array}{l}\text { Adolescent-Focused } \\
\text { Stress }\end{array}$ & $113.74 * *$ & 28.34 & 109.25 & 28.71 \\
\hline Parent-Focused Stress & 82.34 & 17.91 & 80.54 & 22.81 \\
\hline $\begin{array}{l}\text { Adolescent-Parent } \\
\text { Relationship }\end{array}$ & 38.74 & 11.77 & 38.71 & 10.23 \\
\hline Life Stressors & 3.29 & 2.21 & 3.92 & 2.48 \\
\hline \multicolumn{5}{|c|}{ *Borderline Clinical Range on the CBCL (>65) } \\
\hline
\end{tabular}

A PDA was conducted $(n=59)$ to examine the degree to which 10 predictor variables could create a predictive model of adolescent persistence in counseling. The overall Wilks’ lambda was statistically significant $(\Lambda=.70, \mathrm{p}=.03)$. Examination of the classification results matrix provides an understanding of the overall predictability, or "hit rate," of the model (Huberty \& Olejnik, 2006). A model with a high hit rate provides a framework to predict whether an adolescent client will persist in or prematurely terminate from counseling. The hit rate for the model was $74.6 \%$, meaning that 44 of the 59 cases were correctly classified based on the model. Of the clients who persisted in counseling, 75\% $(n=18)$ were predicted correctly; $74.3 \%(n=26)$ of adolescents who prematurely terminated were predicted correctly. These hit 
rates are greater than what would be expected by chance (prematurely terminated 59.3\%, persisted, $40.7 \%)$.

Classification function coefficients serve as the linear classification function for the classification of future cases (Huberty \& Olejnik; 2006). Multiplying the score of each predictor by the computed weight, adding the resulting products, and then adding the constant value achieve a linear composite score for a case. This computation results in two composite scores, with future cases being assigned to whichever group has the largest score (Huberty \& Olejnik, 2006). See Table 5 for the classification function coefficients.

Table 5

Classification Function Coefficients

\begin{tabular}{lcc}
\hline & \multicolumn{2}{c}{ Groups } \\
\cline { 2 - 3 } & $\begin{array}{c}\text { Premature } \\
\text { Termination }\end{array}$ & Persist \\
\hline Gender & 3.876 & 5.866 \\
Age & 6.079 & 5.580 \\
Adolescent involvement in intake & -1.184 & -.753 \\
Internalizing Behaviors & .455 & .527 \\
Externalizing Behaviors & .531 & .522 \\
Parent-Focused Stress & -.031 & -.049 \\
Adolescent-Focused Stress & -.049 & -.047 \\
Adolescent-Parent Relationship Stress & -.032 & -.033 \\
Life Stressors & -.466 & -.305 \\
(Constant) & -70.387 & -67.952 \\
\hline
\end{tabular}

\section{Discussion}

The variables examined in this study were derived from an examination of previous research on counseling persistence. All analyses conducted provided critical information regarding persistence in counseling for counselors and counseling agencies who provide services to adolescent clients and their families. The regression revealed that the predictors examined 
accounted for $29.1 \%$ of the variance in the number of counseling sessions attended by the adolescent clients. Further, the predictive model was able to identify the likelihood of treatment persistence for three quarters of adolescents in this sample. Adolescents face an array of barriers in seeking out counseling, and the National Alliance on Mental Illness (2013) found that only $20 \%$ of youth struggling with mental illness received appropriate treatment for their mental health concerns. When adolescents and their families seek out counseling, it is imperative for counselors to be able to provide support to ensure adolescents are able to persist.

The most salient factor that emerged from the regression analysis was externalizing behaviors, which accounted for over $40 \%$ of the variance explained in number of sessions attended by adolescent clients. Additionally, there was a negative relationship between the variables, suggesting that adolescents with high levels of externalizing behaviors may be at risk of early termination. While not as striking the relationship with externalizing behaviors, internalizing behaviors accounted for $12.96 \%$ of the variance in the predicted number of sessions attended. These findings are consistent with those of researchers who found that internalizing behaviors such as depression and anxiety were indicative of treatment persistence but externalizing behaviors such as delinquency and substance abuse were indicative of premature termination (Baruch et al., 2009; Pagnin et al., 2004; Sunseri, 2003). An understanding of adolescents’ presenting concerns, whether externalizing or internalizing in nature, serve as a valuable assessment tool in assessing client's ability to persist in counseling.

The regression analysis revealed that adolescent involvement in the intake accounted for nearly one-third of the variance explained in number of counseling sessions attended, with adolescents involved in intake attending more sessions. Although clinic protocols included advising parents of adolescents to bring their adolescent child to the intake session, less than a 
third of adolescents were actually involved in the initiation of their counseling services. While only minor differences were observed between adolescents who persisted in counseling and those who prematurely terminated from counseling, the overall low rate of involvement is meaningful from a clinical standpoint. Two clinical issues arise from this: lack of clinical information provided by the adolescent, and lack of perceived involvement in the counseling process by the adolescent. Adolescence is a time of increased autonomy and profound changes in the parent-adolescent relationship (Silverberg \& Steinberg, 1990). Counselors and counseling centers that provide services to adolescents may wish to review their policies on early involvement of adolescents in their counseling process. Early involvement would allow adolescents to provide counselors with valuable clinical information. Additionally, it would provide adolescents with opportunity to be engaged in the counseling process rather than feeling as though they are being sent involuntarily. Early engagement would also provide opportunity for development of the therapeutic relationship, decreasing the risk that young clients view counselors as extensions of their parents.

In previous studies examining persistence in child and adolescent counseling, researchers found high levels of parental stress to be associated with premature termination (Attride-Stirling, et al., 2004; Kazdin \& Mazurick, 1994; Kazdin et al., 1995; Prinz \& Miller, 1994; Tsai \& Ray, 2011). Although the levels of parental stress in the current study were generally higher for adolescents who prematurely terminated, adolescent-focused stress for adolescents who prematurely terminated from counseling was the only parental stress score in the borderline clinical range (Sheras et al., 1998). Results from the regression analysis revealed that life stressors and adolescent-parent relationship were positively correlated with the number of sessions attended, while parent-focused stress and adolescent-focused stress were negatively 
correlated with the number of sessions attended. Further, parent-focused stress accounted for $10.30 \%$ of the variance explained in the number of sessions attended, indicating adolescents with parents who were experiencing high levels of stress related to their parental role attended fewer counseling sessions. Overall, the findings from the current study were consistent with previous research; parent stress can be a limiting factor in adolescent persistence in counseling (AttrideStirling, et al., 2004; Kazdin \& Mazurick, 1994; Kazdin et al., 1995; Prinz \& Miller, 1994; Tsai \& Ray, 2011). Thus, it is imperative for counselors to provide appropriate support and resources to parents to increase the likelihood that adolescents will continue in counseling.

In this sample, the average time spent on the wait-list for adolescents who prematurely terminated (71.86 days) appeared substantially longer than the time spent on the wait-list for adolescents who persisted in counseling (57.00 days). Services provided at the clinics were based on a sliding-scale fee, which may have contributed to the extended wait times across both groups. Unlike Long et al.’s (1992) findings on adult persistence in counseling, the length of time on waitlist did not appear to be a significant factor is explaining the number of sessions attended. It is unknown how this factor impacted families' decisions to engage in counseling. Clients may have sought services elsewhere, the distress they experienced may have became more manageable, or they may have been discouraged with services and chose to not engage in counseling. Because clients often seek out counseling in times of crisis, it is imperative for counselors and clinics to be transparent with clients and their families about the potential length of time they will need to wait prior to receiving counseling services. In order to ensure that counselors are acting with beneficence, counselors must also provide adequate resources and referrals to clients if they are unable to provide timely services. 
An overall examination of the predictor variables revealed the following trends.

Adolescents who are older, male, not engaged in their intake session, present with high levels of externalizing behaviors, or whose parents are experiencing high levels of stress are at a greater risk for prematurely terminating from counseling. Conversely, adolescents who are younger, female, engaged in their intake session, present with high levels of internalizing behaviors, and whose parents are not experiencing high levels of stress are more likely to persist in counseling. The purpose of a PDA is to create a predictive model that can be utilized by counselors to identify adolescents who are at risk of prematurely terminating from counseling. Specifically, the current model serves to predict whether an adolescent client will attend either only the intake session and/or the first counseling session or persist through at least eight counseling sessions, which has been identified as being the point clients begin to experience therapeutic change (Lambert, 2013). While this model is useful as an additional screening tool, it is not without error and should not replace counselor's clinical judgment in identifying clients at-risk of prematurely terminating. Continued examination of the model may provide additional insight into the most predictive variables, thus simplifying the assessment procedure.

Overall, findings from the current study are consistent with previous research on the relationship between the nature of clients’ presenting problems on persistence in counseling but was not supportive of previous findings on the impact of parental stress. The early identification of adolescents who present as male, report high levels of externalizing behaviors, or are from homes characterized by high levels of parental stress provide counselors with key identifiers to engage in early-intervention strategies to increase persistence in counseling. Further, the predictive model created may serve as a screening tool to identify adolescents who are at risk of premature termination from counseling. 
Implications for Clinical Practice

For counselors working with adolescents, several direct clinical implications emerge from the current findings. These implications range from the initial assessment procedures and continue through the counseling process. Additional implications for family support are also discussed.

The process of an adolescent receiving counseling services typically begins with their parents scheduling an initial intake session. For counselors, this is the first opportunity to encourage early-involvement of the adolescent in the counseling process. Although still legally minors, adolescents are beginning to have increased autonomy and responsibility, and their inclusion in the intake process provides counselors with the opportunity to promote this developmental process. It is essential for counselors to be intentional in collecting clinical information and assessments from not only parents, but also adolescents during the intake session. This serves two purposes: to increase the likelihood of getting an accurate clinical understanding of the presenting concerns and to communicate the adolescent's personal investment in the counseling process. The early establishment of mutual trust and respect may serve to foster the development of the therapeutic relationship and encourage persistence in counseling.

Counselors may also find it helpful to incorporate the predictive model developed in the current study into their assessment procedures to inform their understanding of clients who may be at risk for prematurely terminating from counseling. Dependent on the assessments utilized, if all clinical variables are available, counselors may complete the following steps to determine which group the adolescent client most closely fits (see Table 5): (1) Multiply the score of each predictor by the computed weight, (2) Add the resulting products, (3) Add the constant value 
achieve a linear composite score for a case. This computation results in two composite scores, with future cases being assigned to whichever group has the largest score (Huberty \& Olejnik, 2006).

Further, the identification of clients who are at risk for prematurely terminating from counseling may provide counselors with the ability to engage in early-intervention strategies. These strategies may include identifying counselors for parents, holding parent support groups, or providing resources for other external support. Researchers also found parental engagement to be an important factor in their commitment to the counseling process (Kazdin et al., 1997). The inclusion of parent-consultations and the incorporation of psychoeducation may provide parents with the support needed to adhere to counseling. Counselors may also choose to share the information derived from the model with the adolescent and their parents to discuss the identified risk factors and work collaboratively to address any barriers to treatment.

\section{Limitations and Suggestions for Future Research}

Due to utilization of archival data, the instruments analyzed were pre-determined and administered to the clients. Parents or guardians completed all information and assessments based on their knowledge of the adolescent's behaviors or experiences. No information was available based on adolescents’ personal perception of their concerns. Rescorla et al. (2007) found that adolescent self-report measures revealed higher levels of distress than measures completed by parents. Self-report measures are also subject to social desirability. Future researchers may examine assessments completed by adolescent clients, as well as examine other means of information, including the content of counselor progress notes or counselor characteristics. 
The utilization of archival data also resulted in a limited sample size. Although more adolescent files were identified, a large number of the files were ineligible for inclusion due to incomplete data or assessment protocols. A larger sample size may increase power of the predictive model and provide additional insight into differences between adolescents who prematurely terminate from counseling and those who persist in counseling.

The predictive model did not examine the characteristics of adolescents who did not meet criteria for premature termination or persistence. These clients, who attended between two and seven sessions, fell into a middle group that was not considered to have persisted to the point of significant benefit from treatment (Lambert, 2013). Further analysis of these adolescents was also limited by small sample size $(n=13)$. Additional research on trends of attrition and clinical improvement is needed to provide future researchers with guidance on standardization of terms.

Generalizability of findings is limited because counseling services were provided at a training clinic with no standardized treatment approach, which may have impacted the results of the study. Experience of the counselors ranged from master's level interns to doctoral level practitioners. Researchers have explored the impact of counselor characteristics on client's experience of counseling and found conflicting results in regard to counsel's level of experience and treatment persistence (Lampropoulos et al., 2009; Long et al., 1998). An examination of adolescent client files from a range of treatment facilities, including agencies and private practices, would provide additional information regarding the predictability power of counselor and counseling variables when examining adolescent treatment persistence.

Although the current model was developed based on previous literature on attrition, it is possible that other variables, such as counselor characteristics or perceived available social support, may also be valuable in understanding treatment persistence. Future researchers may 
examine the impact of these other variables in determining the most efficient model to predict persistence in counseling.

\section{Conclusion}

Adolescents often experience distress during this critical developmental period and may benefit from counseling services. Beyond barriers experienced in seeking out counseling, a substantial portion of clients terminates prior to experiencing positive change. Results from the current study indicated that the presence of externalizing behaviors may be the strongest indicator of adolescents who are at risk of prematurely terminating counseling. Involving adolescents in the intake may increase persistence in counseling as well as provide additional clinical information to counselors to accurately assess the adolescent's presenting concerns. The utilization of the predictive model may serve as a tool for counselors to aid in the early identification of adolescents who are at risk for premature termination.

\section{References}

Achenbach, T. M., \& Rescorla, L. (2001). Manual for the ASEBA school-age forms and profiles. Burlington: University of Vermont, Research Center for Children, Youth, \& Families. American Psychiatric Association. (2000). Diagnostic and statistical manual of mental disorders (4th ed., text rev.). Washington, DC: Author.

Attride-Stirling, J., Davis, H., Farrell, L., Groark, C., \& Day, C. (2004). Factors influencing parental engagement in a community child and adolescent mental health service: A qualitative comparison of completers and non-completers. Clinical Child Psychology and Psychiatry, 9, 347-361. doi:10.1177/1359104504043918

Ausubel, D. P. (2002). Theory and problems of adolescent development ( $3^{\text {rd }}$ ed.). Lincoln, NE: Writers Club Press. 
Belfer, M. L. (2008). Child and adolescent mental disorders: The magnitude of the problem across the globe. Journal of Child Psychology and Psychiatry 49(3), 226-236. doi: 10.1111/j.469-7610.2007.01855.x

Chang, H., \& Saunders, D. G. (2002). Predictors of attrition in two types of group programs for men who batter. Journal of Family Violence, 17(3), 273-292. doi:

10.1023/A:1016057328929

Cohen, J. (1992). A power primer. Psychological Bulletin, 112, 155-159. doi:10.1037/0033 $-2909.112 .1 .155$

Dierker, L., Nergiso, J., Wiseman, R., \& Hoff, D. (2001). Factors predicting attrition within a community initiated system of care. Journal of Child and Family Studies, 10(3), 367-383. doi: 10.1023/A:1012581027044

Enea, V., \& Dafinoiu, I. (2009). Motivational/solution-focused intervention for reducing school truancy among adolescents. Journal of Cognitive \& Behavioral Psychotherapies, 9(2), 185-198.

Faul, F., Erdfelder, E., Buchner, A., \& Lang, A. G. (2009). Statistical power analyses using G*Power 3.1: Tests for correlation and regression analyses. Behavior Research Methods, 41, 1149-1160. doi:10.3758/BF03193146

Field, A. (2009) Discovering statistics using SPSS (3 ${ }^{\text {rd }}$ ed.). London, England: Sage. Fortenberry, J. D. (2013). Puberty and adolescent sexuality. Hormones and Behavior, 64, 280287. doi:10.1016/j.yhbeh.2013.03.007

Harris, P. M. (1998). Attrition revisited. American Journal of Evaluation, 19(3), 293-305. doi: $10.1177 / 109821409801900303$ 
Huberty, C. J., \& Olejnik, S. (2006). Applied MANOVA and discriminant analysis ( $2^{\text {nd }}$ ed.). Hoboken, NJ: Wiley-Interscience. doi:10.1002/047178947X

Kadera, S. W., Lambert, M. J., \& Andrews, A. A. (1996). How much therapy is enough? A session by session analysis of the psychotherapy dose-effect relationship. Journal of Psychotherapy Practice and Research, 5(2), 132-151.

Kazdin, A. E., Holland, L., \& Crowley, M. (1997). Family experience of barriers to treatment and termination from child therapy. Journal of Consulting and Clinical Psychology, 65(3), 453-463. doi:10.1037/0022-006X.65.3.453

Kazdin, A. E., \& Mazurick, J. L. (1994). Dropping out of children psychotherapy: Distinguishing early and late dropouts over the course of treatment. Journal of Consulting and Clinical Psychology, 62, 1069-1074.

Kazdin, A. E., Stolar, M. J., \& Marciano, P. L. (1995). Risk factors for dropping out of treatment among white and black families. Journal of Family Psychology, 9(4), 402-417. doi:

10.1037/0893-3200.9.4.402

Lambert, M. J. (2013). The efficacy and effectiveness of psychotherapy. In M. J. Lambert (Ed.), Bergin and Garfield's handbook of psychotherapy and behavior change (6 $6^{\text {th }}$ ed., pp. 169218). New York, NY: Wiley.

Longo, D. A., Lent, R. W., \& Brown, S. D. (1992). Client variables in the prediction of client motivation and attrition. Journal of Counseling Psychology, 39(4), 447-452. doi: 10.1037/0022-0167.39.4.447

Merikangas, K. R., Nakamura, E. G., \& Kessler, R. C. (2010). Epidemiology of mental disorders in children and adolescence. Dialogues in Clinical Neuroscience, 11, 7-20.

National Alliance on Mental Illness (2013). Mental illness facts and numbers. Retrieved from 
http://www.nami.org/factsheets/mentalillness_factsheet.pdf

Pagnin, D. de Queiroz, V., \& Saggese, E. G. (2004). Predictors of attrition from day treatment of adolescents with substance-related disorders. Addictive Behaviors 30(2005), 1065-1069. doi:10.1016/j.addbeh.2004.09.013

Poulsen, J., \& French, A. (2002). Discriminant function analysis (DA.) [PDF document.] Retrieved from http://userwww.sfsu.edu/ efc/classes/biol710/discrim/discrim.pdf

Prinz, R., \& Milller, G. (1994). Family-based treatment for childhood antisocial behavior: Experimental influences on drop out and engagement. Journal of Consulting and Clinical Psychology, 62, 645-650. doi:10.1037/0022-006X.62.3.645

Reis, B. F., \& Brown, L. G. (1999). Reducing psychotherapy dropouts: maximizing perspective convergence in the psychotherapy dyad. Psychotheapy, 36(2), 123-136. doi: $10.1037 / \mathrm{h} 0087822$

Sheras, P. L., Abidin, R. R., \& Konold, T. R. (1998). Stress index for parents of adolescents. Odessa, FL: Psychological Assessment Resources.

Sherry, A. (2006). Discriminant analysis in counseling psychology research. Counseling Psychologist, 35, 661-683. doi:10.1177/0011000006287103

Sunseri, P. A. (2003). Predicting treatment termination due to running away among adolescents in residential care. Residential Treatment for Children \& Youth, 21(2), 43-60. doi: 10.1300/J007v21n02_03

Tsai, M. H., \& Ray, D. C. (2011). Children in therapy: Learning from evaluation of universitybased community counseling clinical services. Children and Youth Services Review, 33, 901-909. doi:10.1016/j.childyouth.2010.12.011

World Heath Organization. (2014). Prevention of mental disorders: Effective interventions and 
policy options. Geneva, Switzerland: World Health Organization. Retrieved from http://www.who.int/mental_health/evidence/en/prevention_of_mental_disorders_sr.pdf 
APPENDIX A

INTRODUCTION 
Adolescence is a critical time of development, characterized by significant changes that occur as individuals transition from childhood to adulthood (Ausubel, 2002). Changes in adolescents' biological, cognitive, and emotional development can lead to significant mental health concerns and an increase in high-risk behaviors. It is estimated that $13 \%$ of adolescents experience mental illness that significantly impairs their daily functioning, and nearly half of all mental illness experienced by adults begins during adolescence (Belfer, 2008). In a national survey, researchers examined rates of lifetime prevalence rates among adolescents and found nearly $40 \%$ of adolescents met the criteria for anxiety disorders and $20 \%$ met the criteria for conduct disorders based on Diagnostic and Statistical Manual Fourth Edition (DSM-IV; American Psychological Association, 1994) criteria (Merikangas et al., 2009). Further, high-risk behaviors (i.e. substance use, reckless driving) during adolescents contribute to the leading causes of death among this age group (World Health Organization [WHO], 2014). Counseling is effective at decreasing mental health concerns and maladaptive behaviors across the lifespan, with therapeutic change occurring after only eight counseling sessions (Enea \& Dafinoiu, 2009; Kadera, Lambert \& Andrews, 1996; Lambert, 2013).

Although counseling may serve as a protective factor for prolonged emotional concerns, the National Alliance on Mental Illness (2013) found that only one in five youth struggling with mental illness received appropriate treatment for their mental health concerns. Despite the benefits obtained by persisting in counseling, nearly half of all clients terminate prematurely (Wierzbicki \& Pekarik, 1993). Clients who terminate prematurely from counseling are less likely to benefit from treatment than clients who persist in counseling (Prinz \& Miller, 1994). Yap, Reavley, and Jorn (2012) identified several factors as barriers to adolescents seeking treatment, including embarrassment and fear. 
Although attrition continues to be a concern for counselors, few researchers have examined attrition specific to adolescence. Across the lifespan, researchers have examined personal variables (e.g., demographic variables, presenting problems) and environmental variables (e.g., familial characteristics and the counseling process) to further understand characteristics related to client persistence or premature termination from counseling. There is limited knowledge of the specific characteristics that are predictive of adolescents either persisting or prematurely terminating from counseling. What is known is that adolescents are more likely to remain engaged in counseling if they self-identify internalizing behaviors (i.e. feelings of anxiety or depression) or if they seek treatment for themselves (Baruch, Vroura \& Fearon, 2009; Pagnin, de Queiroz, \& Saggese, 2004; Sunseri, 2003). In contrast, adolescents who engage in externalizing behaviors (i.e., delinquency or aggression), or are referred to counseling by an external source (i.e., teachers, judicial system) are more likely to terminate counseling prematurely (Baruch et al., 2009; Pagin et al., 2004; Sunseri, 2003).

Despite the developmental differences between childhood and adolescence, counseling researchers often examine youth simultaneously. When doing so, demographic variables such as age, gender, and ethnicity were not found to be predictive for treatment persistence (Dierker Nergiso, Wiseman \& Hoff, 2001). In comparison to the findings of the adolescent studies, researchers found conflicting results for externalizing behaviors in children (Dierker et al., 2001; Kazdin, Holland, \& Crowley, 1997). Parental influences also played a significant role in children and adolescents' persistence in counseling. Although client demographic variables were not predictive of premature termination, familial demographic variables such as socioeconomic status (SES) and ethnicity were predictive of premature termination. Adolescents from families whom were headed by a single parent, reported low SES, or were from a non-dominant ethnic 
group, were less likely to persist in counseling (Kazdin et al., 1997). Additionally, adolescents' whose parents who engaged in negative parenting styles or did not have strong positive beliefs about the treatment process were more likely to drop out from counseling prematurely (Kazdin et al., 1997; Venable \& Thompson, 1998). Overall, youth engaged in externalizing behaviors, and whose home environments are characterized by non-dominant groups or high levels of parental stress, were at greater risk for prematurely terminating from counseling.

An examination of treatment persistence across the lifespan revealed several other findings. Researchers examining attrition in child counseling found that increased severity of symptoms at the onset of counseling was associated with premature termination (Kazdin \& Mazurick, 1994; Tsai \& Ray, 2011). This increase in premature termination may be associated with the impact on the family system, along with the extended length of treatment needed to experience meaningful change (Kazdin \& Mazurick, 1994; Tsai \& Ray, 2011). Congruent with the research on children and adolescents, parental stress, adverse parenting styles, and parents' beliefs about counseling and their involvement in the process played a significant role in the persistence of their children in counseling (Attride-Stirling, Davis, Farrell, Groark, \& Day, 2004; Kazdin \& Mazurick, 1994; Kazdin, Stolar \& Maciano, 1995; Prinz \& Miller, 1994; Tsai \& Ray, 2011). For children, it is evident that environmental (i.e. parental stress, familial characteristics) impact their persistence in counseling.

Researchers examined persistence in adult clients in two dominant treatment settings: university-based clinics and specialized program for target behaviors. Across studies, adults who reported low SES, had lower levels of education, and identified from non-dominant ethnic groups were more likely to terminate from counseling prematurely (Reis \& Brown, 1999; Wiezbicki \& Pekarik 1993). Clients’ levels of motivation and self-efficacy were associated with 
the likelihood that they would persist in counseling (Chang \& Saunders, 2002). This finding suggests that identifying internal motivators for clients may serve as a protective factor against premature termination.

Across the lifespan, it is evident that a client's motivation to seek treatment and familial support are important factors in clients' abilities to persist in counseling. Although for adolescents, internalizing behaviors were predictive of persistence in counseling and externalizing behaviors were predictive of premature termination from counseling (Baruch et al., 2009; Pagnin et al., 2004; Sunseri, 2003), these findings were inconsistent throughout the lifespan (Dierker et al., 2001; Kazdin et al., 1997). Additionally, households characterized by high levels of parental stress were associated with high rates of attrition (Attride-Stirling et al., 2004; Kazdin \& Mazurick, 1994; Kazdin et al., 1995; Prinz \& Miller, 1994; Tsai \& Ray, 2011). Although research on the lifespan provides additional information on understanding factors that are predictive of client persistence, adolescent persistence remains understudied. Because of this, assumptions are frequently made based on an adolescent's status as being post-childhood and pre-adulthood, without understanding the unique developmental characteristics of this critical developmental period (Ausubel, 2002).

\section{Statement of the Problem}

With the high occurrence of mental health concerns and high-risk behaviors that occur during adolescence, many adolescents may benefit from participating in counseling services. Counselors continue to struggle with clients prematurely terminating from counseling, thus limiting the benefits that can be achieved. Counselors' understanding of the characteristics of adolescent clients and their families that are predictive of their likelihood to persist or terminate from counseling prematurely is limited. Expanding the lens to examine attrition across the 
lifespan provides additional, but limited, knowledge regarding the factors that are associated with persistence in counseling. Without a comprehensive understanding of predictive characteristics, it remains difficult for counselors to identify and support adolescent clients who are at-risk for terminating from counseling prematurely.

\section{Purpose of the Study}

The purpose of the current study was to develop a predictive model of adolescent persistence in counseling. I examined both personal and environmental characteristics of adolescents who sought counseling services. I hoped to understand characteristics of adolescent clients who terminated prematurely compared to those who persisted in counseling.

Questions to be Addressed

Research questions addressed in this study included:

1. How much of the variance in the number of counseling sessions attended by adolescent clients is explained by adolescent involvement in intake, time spent on wait list, levels of parental stress, and parent perceptions of adolescent internalizing and externalizing behaviors?

2. a. What is the demographic and clinical profile of adolescent clients who prematurely terminated from counseling compared to those who persisted in counseling?

b. To what degree can gender, age, whether an adolescent is involved in intake, time spent on wait list, levels of parental stress, and parent perceptions of adolescent internalizing and externalizing behaviors predict whether an adolescent will terminate prematurely or persist in counseling? 


\section{Significance of the Study}

Client persistence in counseling can lead to a decrease in pervasive mental health problems and increase clients' overall levels of functioning (Lambert, 2013). If counselors can better understand characteristics that are predictive of adolescent persistence in counseling, counselors will be able to better identify clients who may be at risk for premature termination. By gaining this knowledge, counselors may be able to develop retention strategies to increase the likelihood of clients persisting in counseling, thus decreasing the likelihood that distress experienced during adolescents manifest in lifelong mental health concerns (Harris, 1998).

\section{Conclusion}

Given the importance of counseling to serve as a protective factor during adolescence, it is important for counselors to be able to identify adolescents at-risk for terminating counseling prematurely. I aim to further expand the literature regarding factors that are predictive of adolescent persistence in counseling. I will thoroughly examine archival data of adolescent client files from a university-based training clinic in the southwestern United States to gain an understanding of the differences between adolescents who persist in counseling and those who prematurely terminate from counseling. In Appendix B, I provide a comprehensive review of the literature including adolescent development; trends in help seeking, and variables associated with attrition across the lifespan. In Appendix C, I discuss the methodology utilized to examine archival data to create a predictive model of adolescent persistence in counseling. Appendix D provides an extended review of the results, and Appendix E discusses the implications for the current findings. Appendix F includes copies of all assessments and forms utilized in data collection. 
APPENDIX B

\section{EXTENDED LITERATURE VIEW}


Adolescence is characterized by sudden and critical developmental changes that occur during the short transitional period between childhood and adulthood (Ausubel, 2002). The World Health Organization (WHO; 2015) defined adolescence as the age range of 10 to 19, but specified that cultural influences impact the recognized norms for a particular society. Steinberg and Morris (2001) explained adolescence "as a time when individuals begin to explore and examine psychological characteristics of the self in order to discover who they really are, and how they fit into the world they live” (p. 91). These changes often lead to the high occurrence of mental health concerns and at-risk behaviors experienced by adolescents. Despite evidence that counseling can reduce the long-term impact of mental health concerns experienced by adolescents, stigmatization related to help-seeking and a belief in self-reliance often result in adolescents navigating their struggles independently (Del Mauro \& Williams, 2012).

Although rates of mental health issues reach nearly $40 \%$ during adolescence (Merikangas et al., 2010), theories explaining the root of prevalence remain inconclusive (Steinberg \& Morris, 2001). Researchers have proposed several theories involving hormonal changes, the occurrence of stressful life events, and the development of cognitive abilities and coping skills (Steinberg \& Morris, 2001). Therefore, it is important to explore the experience of adolescence, including changes experienced during this developmental period and rates of mental health concerns among this population. Moreover, a review of research examining predictors of attrition and persistence in counseling across the lifespan clarifies potentially important factors to consider.

\section{Adolescent Development}

Understanding an individual's developmental stage is essential prior to fully understanding one's behavior or experience (Ausubel, 2002). The WHO (2014) defines adolescence as the developmental period between childhood and adulthood that is characterized 
by profound biological, cognitive, and social changes. This unique developmental period is second only to infancy in the degree and speed of development (Behr, Nuding, \& McGinnis, 2012).

\section{Biological Changes}

During the developmental period of adolescence, dramatic biological changes begin to occur (Lerner, 2002). These changes are often synergistic with the onset of puberty. Arnett (2012) defined puberty as a period of physical and sexual maturity initiated by hormonal changes. Emerging from childhood, adolescents experience physical and biological changes, including changes in their physical appearance and sexual anatomy (Lerner, 2002). Adolescents must find ways to understand changes they are experiencing; this understanding is often derived from their social environment (Arnett, 2012).

Societal messages regarding the changes that occur during puberty play a significant role in the experiences of adolescents (Arnett, 2012). These experiences are often different for males and females and are highly dependent on the timing on onset (Lerner, 2002). For example, females who reach puberty early experience higher levels of distress than males who reach puberty early. In the primary culture of the United States, puberty is not a time of celebration as in other traditional cultures (Arnett, 2012). Adolescents often approach this period of development with uncertainty, making them acutely aware of others' responses to their changes. These interactions can result in significant changes within their interpersonal relationships (Arnett, 2012). One particular relationship impacted is that of adolescent and primary caregiver. During puberty, changes occur within the parent-child relationship, including a decrease in physical closeness and an increase in conflict (Arnett, 2004). These behaviors are particularly evident in parent-child relationships with children who have early onset puberty (Arnett, 2004). 
In addition to the parent-child relationship, the timing of these biological changes also impacts other interpersonal relationships.

Changes within social relationships are highly dependent on the adolescent's perception of the timing of puberty (Lerner, 2002). These changes can be seen in an examination of adolescents’ self- concept and interpersonal relationships. Cultural messages perpetuate idealized body images for young girls, leading girls who reach puberty earlier than their peers to be at higher risk for maladaptive and psychological distress including depression, substance use, adverse body image, and delinquency (Arnett, 2012). Additionally, early onset of girls’ physical development can lead to creation of relationships with older peers, specifically males, which may increase the occurrence of delinquent behaviors including substance use and sexual activity (Arnett, 2012). Conversely, girls who mature later than their peers also struggle with unfavorable body image issues resulting from messages regarding the lack of development in their sexual organs (Arnett, 2012).

For adolescent boys, early maturation often carries substantially different messages from their female peers. Early maturation among males is associated with increased popularity among peers and positive body image (Arnett, 2012). However, similar to female adolescents, early development is associated with increased rates of delinquency, sexual activity, and substance use (Arnett, 2012). Across genders, the timing of puberty, and the messages received by peers, can significantly impact on the well-being of adolescents. In addition to biological changes, adolescents also experience changes within their cognitive functioning, which play a role in the ways in which they function within the world. 


\section{Cognitive Changes}

As adolescents continue towards adulthood, they often face situations that require them to make decisions similar to that of adults. Although adolescents may be faced with adult decisions, their ability to make healthy decisions may not be within their cognitive ability. Arnett (2004) explained that these cognitive changes effect how "young people think, how they solve problems, and how their capacities for memory and attention change” (p. 64). The cognitive development that occurs during adolescence allows adolescents to move towards effective decision-making abilities.

Jean Piaget’s (1964) cognitive development theory examined the transition of childhood through adolescence. Piaget proposed that individuals proceed through a series of stages that distinguish how they process information and see the world. His theory emphasized the importance of an individual's maturation versus the impact on environment on an individual's cognitive development. The period of adolescence falls within the formal operation stage, typically beginning around age 11 and extending through ages 15-20. During this stage, Piaget suggested that adolescents are capable of thinking both logically and abstractly and are able to formulate and test hypotheses. It is adolescents' ability to see alternative options that creates opportunities for adolescents to engage in decisions that lead to maladaptive behaviors (Piaget, 1964). The decisions that adolescents face become increasing complex. Although adolescents are increasingly capable of engaging in abstract thought, it remains difficult for them to make decisions that decrease the likelihood of negative consequences.

Changes in Emotional Development

Exploration of self is a significant task during adolescence (Lerner, 2002). The ability to self-reflect emerges as children move into adolescence (Arnett, 2004). Erikson (1968) explained 
that adolescence is a period of self-exploration resulting from the manifestation of an identity crisis. He suggested that during this developmental period, the task of fidelity encourages adolescents to explore the facets of their identity and their roles within society. It is often common for adolescents to differentiate their sense of self, dependent on the social situation or relationship (Steinberg \& Morris, 2001). For example, one’s identity may be different when interacting with peers versus with one's parents or with self. This differentiation may cause psychological distress within the adolescent or within his or her relationships.

An additional role that adolescents explore is their sexual identity (Lerner, 2002). Adolescents must find ways to manage their emotional responses and embark in finding socially and personally acceptable ways to deal with their sexuality (Lerner, 2002). Their exploration of sexuality impacts their personal development as well as their relationships with family and peers (Beharry, 2013). Four key elements of sexuality during adolescence have been identified: desire, arousal, behaviors, and functioning. These include engagement in sexual exploration both interpersonally and interpersonally. Each of these areas of exploration can have a profound impact on adolescents’ development (Fortenberry, 2013).

Adolescents experience feelings of fear and confusion as a result of these changes (Lerner, 2002). Ausubel (2002) explained, “psychological disturbances in adolescence are exceedingly common and often appear extreme in nature, serious, and alarming” (p. 547). Due to the profound changes that occur in their biological, psychological, and social worlds, adolescence can be a time of emotional distress even for those that previously did not experience difficulties. Developmental changes during adolescence can have expansive social and psychological consequences (Ausubel, 2002). Internalizing behaviors, such as depression and suicide, tend to increase throughout the developmental period of adolescence (Steinberg \& 
Morris, 2001). Because of this, adolescents experience high rates of mental health concerns and a significant shift in their relational needs (Ausubel, 2002).

Relational Changes

Beyond the changes experienced in relationships as a result of pubertal and cognitive changes, the period of adolescence is also characterized as a time of exploration within relationships. Adolescents begin to seek out relationships to that allow them to explore their identity and fulfill their changing emotional needs. This exploration impacts adolescents' social identity as well as their role within the family system.

As adolescents transition from childhood, their attachments begin to shift from their parents to their peers and other non-familial groups (Steinberg \& Morris, 2001). Consequently, adolescence is often a time of dissention in the parent-child relationship, characterized by an increased occurrence of conflict (Ausubel, 2002; Steinberg \& Morris, 2001). Perceptions of quality of communication between parents and adolescents are incongruent. For example, Smetana, Metzger, Gettman, and Compione-Barr (2006) assessed 276 adolescents in the $9^{\text {th }}$ and $12^{\text {th }}$ grades, along with their parents, to understand rates of disclosure by adolescents to their parents across three major life domains: personal, school, and peers. They found that adolescents were more likely to discuss issues related to school life than issues related to their personal lives, and were more likely to discuss these issues with their mothers than their fathers, regardless of gender (Smetata et al., 2006). The lack of observed willingness by adolescents to engage in conversations regarding their personal lives has the potential to magnify the occurrence of experienced distress. The striving for autonomy that occurs during adolescence affects not only the psychological development of the adolescent, but also the psychological well-being of 
parents (Silverberg \& Steinberg, 1990). The distress experienced by parents may serve as a catalyst for parents to seek counseling services for their adolescents.

As adolescents' focus shifts to other non-familial relationships, the characteristics of these relationships can have a profound impact on their experiences during these transitional years. Contrary to the often-held societal belief that peer groups only contribute to negative behaviors, researchers found that peer groups also support positive, or pro-social behaviors (Steinberg \& Morris, 2001; Wentzel \& Caldwell, 1997). Relational changes during adolescence also impact choices in who, or how, to seek help for struggles.

Mental Health Concerns in Adolescence

The sudden and critical developmental changes that occur during the adolescent years can have a profound impact on an individual's ability to achieve and maintain a healthy homeostatic state (Ausubel, 2002). Researchers have extensively examined the presence of pathology and maladaptive behaviors in adolescence. A national survey found approximately one in five adolescents met the criteria for a mental health disorder (Kessler Berglund, Demler, Jin, Merikangas \& Walters, 2005). Additionally, a review of epidemiological data revealed nearly $50 \%$ of mental illness experienced by adults begins during adolescence (Belfer, 2008). Thus, it is important to explore the occurrence of mental health concerns among adolescents.

Merikangas, Nakamura, and Kessler (2009) conducted an epidemiological review of mental health concerns among children and adolescents. From their review, they concluded that one out of every four youth struggled with a mental disorder in the past year. Among the highest rates of occurrence are depression and anxiety disorders. Longitudinal studies identified the age of onset for major depressive disorder (MDD) often lies between ages of 11 and 14, coinciding with the transition from childhood to adolescence (Merikangas et al., 2009). Across studies, 
rates of adolescent anxiety ranged from 2.2 to 9.5\% (Merikangas et al., 2009). Emerging from childhood, overanxious disorder appeared to be the most prevalent, but during adolescence, social phobias and panic disorders remained the most prevalent. As adolescents transition into young adulthood, generalized anxiety disorder (GAD) emerged as the most prevalent form of anxiety disorders (Merikangas et al., 2009). Additionally, the occurrence of MDD and bipolar disorder are correlated with the presence of many other disorders in youth, including attention deficit/hyperactivity disorder (ADHD), anxiety disorders, oppositional defiant disorder (ODD), and conduct disorder. This association was further supported by the identification of adolescent onset of anxiety and depression as indicators of bipolar disorder in adulthood (Merikangas et al., 2009).

Researchers conducted a national survey to examine feelings of depression and suicidal ideations among high school students $(\mathrm{N}=15,400)$. They found that nearly $30 \%$ of adolescent participants reported that they stopped engaging in usual activities due to feeling sad or hopeless nearly everyday for two or more weeks during the past year (U.S. Department of Health and Human Services, 2012). Over 15\% of students reported seriously considering suicide, and 7.8\% reported having attempted suicide at least once in the past year. In addition to the high rates of suicide attempts, suicide was found to be the third leading cause of death among adolescents (WHO, 2014). This indicates that nearly one out of every three adolescents is struggling with a serious mental health concern that can significantly impact their current and long-term functioning or even lead to death.

Researchers often focus on the differences between genders in externalizing and internalizing behaviors. In a multi-country epidemiological study, Rescorla, Achenbach, Almqvist, and Bird (2007) examined the presence of emotional and behavioral problems for 
adolescents $(N=27,206)$ from 24 countries. The researchers utilized the Youth Self Report (YSR; Achenbach \& Rescorla, 2001) and found that, consistent with Western societal beliefs, adolescent males displayed significantly higher rates of externalizing behaviors than adolescent females (Rescorla et al., 2007). In this study, externalizing behaviors were characterized by Rule Breaking and Aggressive Behavior and the DSM-oriented scale of Conduct Problems. The occurrence of externalizing behaviors, which are characteristic of adolescent males, can lead to social and personal consequences for their high-risk behaviors. Conversely, adolescent females displayed significantly more internalizing symptoms than their male counterparts (Rescorla et al., 2007). On the YSR, internalizing behaviors included Anxious/Depressed, Withdrawn/Depressed, and Somatic Complaints and included DSM-oriented scales that indicate Affective, Anxiety, and Somatic Problems (Rescorla et al., 2007). Although different, the experience of both externalizing and internalizing behaviors are indicative of mental health concerns during adolescence.

The presence of mental health concerns among adolescents has a profound and lifethreatening impact. According to the WHO (2014) the leading causes of death among adolescents are due to injuries sustained on the road, contraction of HIV, suicide, respiratory infections, and interpersonal violence. An examination of these causes reveals that all but one (respiratory infections) are related to risk-taking behaviors and mental health.

The U.S. Department of Health and Human Services (2012) conducted a national survey of high school students to examine the occurrence of high-risk behaviors that led to greatest number of deaths among adolescents. The researchers utilized a three-stage sample cluster method to capture a nationally representative sample. Adolescents in grades 9 through 12 from 158 schools completed over 15,400 surveys. The Youth Risk Behavior Surveillance Survey 
(YRBSS) consisted of 86 questions and assessed the occurrence of "health-risk behaviors" across six domains: (1) behaviors that contribute to unintentional injuries or violence; (2) tobacco use; (3), alcohol and other drug use; (4) sexual behaviors that contribute to unintended pregnancy and sexually transmitted diseases; (5) unhealthy dietary behaviors; and (6) physical activity (U.S. Department of Health and Human Services, 2012). Results indicated that many youth are engaged in high-risk behaviors that can have a profound impact on their physical and mental health.

Adolescents often face pressures to engage in high-risk behaviors, including substance use, risky sexual encounters, and reckless driving, which can all result in long-term consequences (WHO, 2014). Unlike internalizing behaviors, externalizing behaviors tend to peak during middle adolescent years and continue to decline as adolescents transition to adulthood (Steinberg \& Morris, 2001). The YRBSS (U.S. Department of Health and Human Services, 2012) also measured the lifetime substance use rates of the adolescent participants. Adolescents reported the following rates of substance use: alcohol (70.8\%), marijuana (39.9\%), prescription drugs without a doctor's approval (20.7\%), inhalants (11.4\%), hallucinogens (8.7\%), ecstasy (8.2\%), cocaine (6.8\%), methamphetamines (3.8\%), and heroin (2.9\%). Due to the profound impacts related to substance use, ranging from health concerns to legal implications, substance use remains a primary concern during adolescence. High-risk sexual behaviors were also assessed. Nearly $40 \%$ of adolescents reported not using protection during their last sexual intercourse. Externalizing behaviors during adolescence can lead to significant unhealthy consequences for adolescents. Thus, it is important for counselors to understand differences among genders in order to provide adequate support. 
Despite the research examined, Belfer (2008) warned that, for children and adolescents, there are several factors that have the potential to reduce the reported rates of mental health disorders. Often, problems are identified and managed within a school or other non-clinical setting. Additionally, although many researchers gathered information from parents or caretakers, when given self-report measures, adolescents reported significantly more problems than those reported by their parents (Rescorla et al., 2007). Although researchers have been able to identify high rates of distress during adolescence, counselors should be mindful that actual prevalence rates may be even more significant.

The biological, cognitive, and emotional changes that occur during the period of adolescence have an impact on adolescents' mental health and relationships. Nearly $20 \%$ of adolescents meet the criteria for a mental health disorder, and suicide remains the third leading cause of death among adolescents (Kessler et al., 2005; WHO, 2014). To reduce the risk of mental health concerns having a profound impact on adolescents' long-term wellness, it is imperative to understand the means by which they seek support.

\section{Adolescent Help Seeking}

Whether adolescents choose to seek support for their mental health concerns, or choose to deal with them autonomously, may have a significant impact on their long-term functioning. Many factors contribute to adolescents' decisions to seek help, including available support systems and perceived source of their concern. Further impacting their decision is the degree of awareness they hold regarding the significance of their current experiences on their long-term well-being.

Severity of emotional distress does not appear to be positively correlated with help seeking among adolescents. Husky, McGuire, Flynn, Chrostowski, and Olfson (2009) assessed 
364 high school students for presence of mental health problems and their willingness to request help. Of the students who screened positive for emotional distress on the Columbia Health Screen $(n=83), 73.5 \%$ requested follow-up support. Over a quarter of the students chose not to seek help, and among these students, nearly $60 \%$ of them reported having suicidal ideation in the past three months and $45.4 \%$ reported a previous suicide attempt (Husky et al., 2009). Of the students that tested positive and did not request help, there was a statistically significant difference $(p<.05)$ among demographic variables. These students were mostly White $\left(x^{2}=\right.$ 10.38) had higher grades $\left(x^{2}=2.51\right)$, fewer absences $\left(x^{2}=-2.16\right)$ and detentions $\left(x^{2}=-2.27\right)$. Adolescents exposed to violence were also unlikely to seek professional support for their victimization (Husky et al., 2009). Although the majority of adolescents who were experiencing distress sought out additional resources, students who were experiencing high-levels of distress, marked by suicidal ideations or attempts, were less likely to be receptive of services. Additionally, it appeared that adolescents who were experiencing emotional distress but were experiencing limited educational consequences were less likely to seek help.

The National Longitudinal Survey of Adolescent Mental Health surveyed 4,590 adolescents on their utilization of mental health services and exposure to violence (Guterman, Hahm, \& Cameron, 2002). Adolescents indicated if they had been jumped, had a knife or gun pulled on them, or were stabbed or shot in the past year. Nearly $20 \%$ of the respondents reported being personally victimized by one or more violent acts within the past year. Only $11 \%$ of these adolescents had received professional mental health services for their victimization, leaving nearly $90 \%$ of adolescents to handle these traumatic experiences without professional support. These trends indicate that although students were struggling with significant emotional distress, it was difficult for parents and mental health professionals to identify at-risk students. 
Counselors know relatively little about the actual referral rates of adolescents, but it is apparent that in many instances, the choice to seek help from a mental health professional does not lie in the hands of the adolescent. Often, this decision may be made by those impacted by the adolescent's choices or behaviors; this may include distressed parents, frustrated teachers, or even the judicial system. Adolescents demonstrate little awareness of how their current experiences may impact their long-term well-being (Siegel, Cousins, Robovits, Parsons, Lavery, \& Crowley, 1994). Across the lifespan, clients who are mandated to counseling have shown comparable outcomes to clients who voluntarily engage in counseling (Bastien \& Adelman, 1984; Snyder \& Anderson, 2009). Therefore, despite how clients arrive to counseling, positive treatment outcomes remain achievable. Although minimal research exists examining the experiences of adolescents who enter treatment involuntarily, researchers suggested that counselors' early engagement of adolescent clients in the treatment process significantly increased clients' motivation and perceived treatment effectiveness (Tan \& Fegert, 2004). Sources of Support

Adolescents are more likely to seek help from informal sources of support such as friends and family, than from formal helping professionals (Del Mauro \& Williams, 2012). Despite the discord in parent-child relationships that is often characteristic of adolescence, researchers found that adolescents continue to rely on that relationship for support (Del Mauro \& Williams, 2012; Fallon \& Bowles, 2001; Yap et al., 2012). An examination of the literature related to adolescent help-seeking revealed conflicting results when examining the primary source of informal support, some researchers suggested parents remain the largest source of support while others found peers became the largest source of support for adolescents (Del Mauro \& Williams, 2012; Fallon \& Bowles, 2001; Yap et al., 2012). 
Beginning in childhood, parents serve as models for how to cope with distress, and how to communicate with others when faced with challenges. As children transition to adolescence, characteristics of their relationships with parents impact how they approach their concerns. Fallon and Bowles (2001) surveyed 196 adolescents to understand the relationships between help-seeking behavior and perceived family functioning. The researchers found a negative correlation between time spent with parents and help-seeking from an external source. Fallon and Bowles (2001) proposed this decrease in help seeking behavior could be due to the parent-child relationship serving as a protective factor for adolescents, reducing the need for adolescents to seek professional counseling. In contrast, adolescents who reported higher levels of familial stress were more likely to seek help from non-familial supports, supporting Fallon and Bowles’ (2001) suggestion of the importance of the parent-child relationship as a primary source of support for adolescents. The results also indicated that there was no correlation between the problem type and help-seeking. The reported problems adolescents sought help for were stressors related to family (31\%), relationships (21\%), health (21\%), education (15\%), and financial-legal (12\%). Family related problems were most frequently related to seeking help from a mental health professional, suggesting that distress within the family system impede adolescents from seeking support from parents.

As peers begin to play a more integral role in the lives of adolescents, their role in providing support also increases. Adolescents begin to rely on the support of their peers above that of their parents (Del Mauro \& Williams, 2012; Fry, Rickert, O’Connor, Palmetto, Lessel \& Davidson, 2013). An examination of 1,312 adolescent youth revealed that of those who sought help, 72\% first disclosed their struggle to a peer (Fry et al., 2013). This suggests that nearly twothirds of adolescents are not seeking appropriate professional help for their struggles (Fry et al., 
2013). Although informal sources of support may serve an important role in adolescents' ability to cope, many adolescents may not have these sources available, or are faced with concerns that require the support of counselors.

\section{Barriers to Help Seeking}

Although the WHO (2014) found treatment to reduce the likelihood of increased severity of mental health problems as adolescents move into adulthood, many adolescents experience barriers to obtaining appropriate help. Knowledge regarding mental illness and the mental health profession does not appear to contribute to the lack of help seeking by adolescents (Del Mauro \& Williams, 2012). Adolescents reported they typically acquire this information from their health classes and from previous experiences in receiving counseling services. Instead, the largest barrier lies in how adolescents perceive their current problems (Boldero \& Fallon, 1995).

Adolescents are more likely to seek help when they see the cause of the identified problems as external rather than internal (Del Mauro \& Williams, 2012). Del Mauro and Willians (2012) examined youth's perceptions towards mental health through the utilization of focus groups. They found that all participants $(N=31)$ reported a strong opinion against seeking out professional mental health services. Instead preferring to be self-reliant and handle their problems individually (Del Mauro \& Williams, 2012). However, adolescents attested that in instances of severe mental health concerns (e.g., schizophrenia), they were more open to seeking help than when the problems were perceived as less severe (e.g., mild depression). A major barrier to seeking help included fear that what they shared would not be kept confidential, and would lead to stigmatization and judgment. To avoid stigma and judgment, adolescents chose to be self-reliant and handle problems without external support. Although they held negative views regarding professional help, adolescent participants declared that it was "okay” for others, 
including their friends and family, to seek help for mental health concerns (Del Mauro \& Williams, 2012). Thus, barriers remain that prevent adolescents from seeking help, including a sense of self-reliance and avoidance of social stigma.

Yap et al. (2012) also examined barriers to seeking treatment in youth, which included elements of social stigma. Researchers telephone surveyed 3,021 Australian youth aged between 15 and 25 and utilized vignettes depicting an array of mental health concerns. Of those surveyed, Yap et al. (2012) found only 10\% of respondents reported "nothing would stop them from seeking help” (Yap et al., p. 259). Embarrassment (27\%) and fear of judgment from the counselor (11\%) were reported as the most salient barriers to help seeking, particularly in the instances of the substance abuse and social phobia vignettes. The social stigma concerns that emerged in Del Mauro and Williams’ (2012) findings were strengthened by adolescents’ reports of decreased barriers to seek formal help if they knew of a friend of family member whom had previously received formal support (Yap et al., 2012). Thus, it is evident that youths' helpseeking behaviors are significantly impacted by negative societal beliefs of mental health services.

\section{Mental Health Professionals}

Due to the societal stigmas associated with mental health and the high-risk behaviors experienced by adolescents, adolescents may often feel judged or ostracized by their parents or peers for their behaviors (Lerner, 2002). Additionally, with nearly half of all mental health problems experienced by adults beginning in adolescence, it is evident that seeking professional help is vital to the long-term well-being of adolescents (Belfer, 2008). Unlike other interpersonal relationships, the therapeutic relationship is unique in its ability to provide adolescents with an environment characterized by genuine empathy and unconditional positive regard, which is 
significant in combating the societal judgments faced outside of counseling (Naar-King, 2011). Despite the high rates of pathology that occur during adolescence, only $20 \%$ of youth who are struggling with mental health concerns received appropriate treatment from a mental health professional (National Alliance on Mental Illness, 2013). These adolescents have either overcome the barriers to seeking treatment, or have been mandated by an external source.

For the $20 \%$ of adolescents that receive treatment for their mental health concerns, significant positive treatment outcomes have been identified (National Alliance on Mental Illness, 2013). Motivational interviewing (MI) remains one of the most widely researched treatment modalities for working with adolescents. Jensen, Cushing, Alyward, Craigs, Sorell, and Steele, (2011) conducted a meta-analysis $(N=21)$ to examine the effect of MI as an intervention for adolescents with problems related to substance use. The researchers found a small, but statistically significant effect size post-treatment (mean $d=.173$ ). Studies that provided data regarding follow-up analysis also found a small, but statistically significant effect size for follow-ups that were less than six months $(n=4$, mean $d=.323)$ and longer than six months ( $n=7$, mean $d=.133$ ). Demonstrating the benefits of counseling for adolescents who utilize substances. Enea and Dafinoiu (2009) examined benefits of counseling for adolescents with high rates of truancy. Compared to the wait-list control group $(n=19)$, adolescents who received eight sessions of counseling $(n=19)$ had a $61 \%$ decrease in truancy. Although these studies were conducted with an array of presenting problems, treatment settings, and duration of treatment, the findings suggest that adolescents who received counseling from a mental health professional had a decrease in emotional concerns at high-risk behaviors.

Even when individuals seek out treatment, counselors must combat high rates of attrition among their clients (Wierzbicki \& Pekarik 1993). Researchers found that clients who terminate 
prematurely are less likely to benefit from treatment than clients who persist in counseling (Prinz \& Miller, 1994). Thus, in order for individuals to truly benefit from the experience, they must persist in treatment. Therefore, it is important to explore the construct of attrition.

$$
\text { Understanding Attrition in Counseling }
$$

Across the literature, the terms attrition, and premature/early termination are used interchangeably but are defined in a variety of ways. Mennicke, Lent, and Burgoyne (1988) stated, “in its broadest sense, premature termination refers to clients leaving treatment before their counselors believe they should” (p. 458). This may either be a specified number of sessions or after meeting desired treatment outcomes (Hatchett, 2004). Hatchett (2004) asserted there is a vast difference between clients who terminate counseling under distress after only a few sessions and those who terminate prior to their planned termination date due to perceived lack of necessity.

How researchers define early termination can significantly impact results obtained from studies examining attrition. Hatchett and Park (2003) further explored the impact of operational definitions on premature termination outcome data. Reviewing 85 files from a university clinic, they found attrition rates that ranged from $17.6 \%$ to $53.1 \%$. Results were dependent on the definition used to analyze the data: clients who did not engage in counseling beyond the intake session (17.6\%), terminated based solely on the counselor's judgment (40.8\%), missed the last scheduled appointment (40.8\%), and terminated prior to the median (4) number of sessions at that clinic (53.1\%).

Wierzbicki and Pekarik (1993) also found significant differences across reported rates of attrition based on the ways in which premature termination was defined. Their meta-analysis examined rates of attrition across 125 studies and found the mean rate of attrition was $46.86 \%$ 
$(S D=22.25)$. However, they found substantial differences among rates when examining how premature termination was operationalized across studies. When premature operation was operationalized as the client not attending a predetermined number of sessions $(M=48.23 \%$, SD $=21.39)$ or based solely on counselor judgment of perceived clinical improvement $(M=48.43 \%$, $\mathrm{SD}=23.59)$, rates of attrition were statistically significantly higher $(\mathrm{F}=3.22, p<.05)$ than when premature termination was operationalized as a client not attending a scheduled session $(M=$ $35.87 \%, \mathrm{SD}=16.47)$. This divergence in results indicates the importance of using caution when comparing rates of attrition across studies.

Adding to the difficulty in defining attrition is the lack of understanding regarding the treatment length needed for most clients to experience clinical improvement. Webb and Widseth (2005) noted that research is inconclusive concerning the number of sessions necessary for clinically significant change, asserting only that as the number of sessions attended increases so does clients' reports of clinical improvement. Lambert (2013) conducted a meta-analysis and found that across the lifespan, most clients show clinically significant improvement within seven sessions, and resolution of presenting problems begins to occur after eight sessions. A previous meta-analysis also supported this finding, indicating $50 \%$ of clients achieve clinically significant improvement after eight sessions of counseling (Kadera et al.,1996).

In a meta-analysis of 125 studies across outpatient treatment settings, Wierzbicki and Pekarik (1993) found an overall rate of premature termination at $46.8 \%$ across the lifespan. In a more recent analysis, rates varied across settings, with rates as high as $77.5 \%$ at university training clinics and $45.2 \%$ at community agencies (Callahan, Aubuchon-Endsley, Borja, \& Swift, 2009). Given the varying attrition rates across studies, it remains clear that counselors continue 
to struggle with having clients persist in counseling. Despite the differing definitions of attrition, alarming rates of attrition remains an evident problem for counselors.

In addition to the impact on clients' treatment outcomes, counselors and treatment centers are also impacted by the high rates of attrition. With many facilities operating from waitlists, clients who fail to show for their sessions occupy valuable counselor appointment times (Kazdin, et al.,1997). Lack of occupied appointments leads to a waste of resources and a decrease in clients receiving appropriate treatment (Kazdin et al., 1997). Frequent premature termination can also lead counselors, especially those in training, to question their clinical abilities. In turn, Hatchett (2011) suggested the issue of premature termination be further explored in counselor training programs, to support counselors in developing approaches for dealing with, or combating, the high occurrence of premature termination.

Over the last three decades, researchers have worked to uncover complexities associated with treatment persistence. Researchers have examined an array of client, counselor, and treatment program characteristics to aide in identifying clients at risk for premature termination. However, results are conflicting across studies (Reis \& Brown, 1999; Wierzbicki \& Pekarik 1993). Understanding factors that contribute to premature termination is essential in the development of client retention strategies (Harris, 1998). In the counseling literature, limited research exists in the area of adolescent attrition. Because of this, examining predictive characteristics across the lifespan may serve as a framework for further understanding adolescent treatment outcomes.

Attrition in Adolescent Counseling

Although attrition continues to be a concern for counselors, few researchers have examined attrition specific to adolescent clients. Researchers examining factors predictive of 
treatment persistence focused on two major domains: personal variables (e.g., emotional stressors, behaviors) and environmental variables (e.g., familial factors, therapeutic relationship).

Personal variables. Personal variables are those that are experienced within or by an individual, such as emotional distress or delinquent behavior. Personal variables are vital to understanding individuals’ decisions to persist or prematurely terminate from counseling. Pagnin et al. (2004) examined dropout rates of 104 adolescents from an intensive outpatient treatment facility in Brazil. In this study, the researchers defined dropout as the point in which the adolescents no longer attended treatment for three consecutive days. Based on a psychiatric evaluation, all clients in their study met DSM-IV criteria for a substance-related disorder (American Psychological Association, 1994). The researchers utilized the Child Behavior Checklist/4-18 (CBCL/4-18; Achenbach \& Rescorla, 2001) and the Youth Self Report (YSR; Achenbach \& Rescorla, 2001) to understand presenting concerns and current level of functioning. Pagnin et al. (2004) found adolescents who self-reported feelings of anxiety or depression were significantly more likely to persist in counseling. An increase of one point on the YSR Anxious/Depression subscale was indicative of an increase of 0.96 (95\% CI = $0.94-$ 0.98) times the number of days that the adolescents were involved in treatment (Pagnin et al., 2004). Inversely, delinquent behaviors, as evidenced by parent reported on the CBCL/4-18, were associated with a decrease in treatment attendance. Specifically, a one point increase on the Delinquent Behavior subscale was indicative of a decrease of 1.09 times the number of days that the adolescents were involved in treatment” (Pagnin et al., 2004, p. 1067). Adolescents who reported higher levels of internalizing behaviors were more likely to persist in counseling than adolescents who presented with externalizing behaviors. An examination of substance use behaviors among adolescents also revealed trends in treatment persistence. Although drug 
severity is correlated to prevalence of use (i.e. marijuana 39.9\% versus cocaine $6.8 \%$; U.S. Department of Health and Human Services, 2012), adolescents who used more severe substances, such as cocaine, were more likely to be inconsistent with treatment attendance than those who used less severe substances such as marijuana (Pagnin, et al., 2004). The researchers proposed that persistence in counseling was linked to adolescents' recognition that continuing counseling led to symptom relief from their self-reported feelings of anxiety or depression. The observed trend was opposite for adolescents who engaged in delinquent or severe substance use, indicating their lack of insight regarding the benefit of symptom reduction.

Similarly, Sunseri (2003) examined rates of attrition for adolescents in a residential treatment facility. Of their sample $(N=7,477), 33.1 \%(n=2,476)$ of participants prematurely terminated from treatment by running away from the treatment facility. Consistent with Pagnin et al's (2004) findings, Sunseri found negative externalizing behaviors such as impulsivity, opposition, stealing, and aggression were predictive of adolescents who prematurely terminated counseling. Additionally, adolescents referred by probation or with other legal misconducts and oppositional behaviors (e.g. stealing, pimping, prostituting, vandalism, property destruction, and physical violence) were more likely to run away from treatment (Sunseri, 2003). This was also true for adolescents who displayed a lack of guilt after misconduct or used substances. Conversely, several protective factors were identified: grief, eating disorders, and suicidal ideations (Sunseri, 2003). Sunseri's findings further build the case those adolescents who engage in externalizing, delinquent behaviors are at a higher risk for prematurely terminating from counseling than adolescents who are experiencing internalizing behaviors.

The period of adolescence is often defined differently across settings and studies. Baruch et al. (2009) examined rates of attrition for 882 clients between the ages of 12 and 21 who 
engaged in weekly counseling at a community-based clinic. Although the researchers examined individuals beyond the ages identified within the current study (12-18 years old), many clients fell within the defined range. In this review of archival data, Baruch et al. (2009) defined premature termination as those who did not complete 21 sessions. Consistent with Pagnin et al. (2004) and Sunseri's (2003) findings, Baruch et al. (2009) found a large statistically significant difference in rates of attrition between adolescents with self-reported anxiety and depression on the YSR Anxious/Depressed scale and those who were not anxious/depressed. In addition, adolescents who struggled with aggression towards others, delinquency, and other behaviors consistent with conduct disorder as evidenced by scores on the YSR Delinquent Behavior scale were more likely to terminate prematurely. There was also a statistically significant difference between adolescents who were self-referred to treatment versus those who were referred by others. These findings highlight the importance of the client's internal motivation in influencing persistence in counseling.

Although Baruch et al. (2009), Pagin et al. (2004), and Sunseri (2003) examined characteristics across treatment settings and modalities, two significant findings emerged. Adolescent clients who presented to counseling for anxiety or depression were more likely to persist in counseling, while those who presented with externalizing-delinquent behaviors, such impulsivity and aggression, were more likely to prematurely terminate from counseling. Thus, it is apparent that persistence in counseling is more common among adolescents who are experiencing internalizing symptoms perhaps for the hope of relief from their symptoms (i.e. depression, anxiety). In addition to internal variables, environmental variables also influence rates of persistence among clients. 
Environmental variables. There is limited research examining environmental variables with adolescent clients, the identified studies examine the influence of family environment and variables related to the counseling experience. Adolescents who live in households characterized by distress were more likely to terminate from counseling prematurely. More specifically, composite family functioning scores were inversely related to premature termination (Sunseri, 2003). Among these factors were relational concerns and housing stability. Adolescent clients with self-reported relationship difficulties with their parent(s) were more likely to prematurely terminate from counseling compared to adolescents who did not report difficulty in their parentchild relationship. Adolescents living in households with married parents were statistically significantly more likely to remain in treatment compared to adolescents who lived with unmarried parents (Baruch et al., 2009). Additionally, homelessness was associated with premature termination (Baruch et al., 2009). It appears as if instability and distress within the family system serve as risk factors for adolescents prematurely terminating from counseling.

In a conceptual article, Blotcky and Friedman (1984) proposed that characteristics of the therapeutic relationship contribute to persistence in adolescent counseling. To date, no studies were identified that examine the impact of therapeutic relationship variables related to adolescent counseling. Although it is a commonly held belief that the quality of the therapeutic relationship serves as a protective factor against client premature termination, adolescents may approach counseling with resistance, impacting the ability to form a therapeutic alliance. Blotcky and Friedman (1984) proposed that resistance to engage in a therapeutic relationship may be due to transference within the relationship. They suggested that adolescents often project their feelings of anger with their parents or others in authority towards the counselor, which may contribute to adolescents prematurely ending the relationship. Premature termination may serve as a means to 
avoid unpleasant feelings that often occur during the counseling process. To lessen this effect, Blotcky and Friedman (1984) suggested that flexibility in the counselor's treatment modality would encourage persistence in treatment by strengthening adolescents' perceptions of the therapeutic alliance and empowering them to engage in treatment.

Although there have been a few studies examining characteristics of adolescents who prematurely terminate from counseling, the majority of these studies examined residential treatment which is inherently different than outpatient, weekly counseling sessions (Pagin et al., 2004; Sunseri, 2003). Across the literature, there is a limited knowledge of characteristics of adolescents who persist in counseling. Despite the known significant developmental differences that occur between childhood and adolescence, some researchers have explored youth attrition as a whole, grouping together child and adolescent clients.

Attrition in Child and Adolescent Counseling

Across the counseling literature, childhood and adolescence are often examined together. Due to the developmental, relational, and social differences that exist between child and adolescent clients, the factors present in counseling are vastly different. It is because of this that Wierzbicki and Pekarik (1993) stressed the importance of examining childhood attrition independently from adulthood.

Personal variables. Similar to the research presented on adolescent clients, characteristics and presenting problems have been researched to understand how they impact treatment persistence for child and adolescent clients. Dierker et al. (2001) aimed to understand differences in individual, familial, and program characteristics for the $66 \%$ of children and adolescents who prematurely terminated from residential treatment. Dierker et al. (2001) examined 117 closed client files for youth who were referred to community-based treatment in 
Connecticut for severe emotional and behavioral problems. For purposes of the study, the researchers defined premature termination as those who did not return to counseling prior to meeting their treatment goals.

An examination of demographic variables revealed no statistically significant difference between children and adolescents who persisted in counseling and those who prematurely terminated from counseling (Dierker et al., 2001). Neither age, gender, nor ethnicity were predictive of attrition. A review of mental health concerns and presenting problems provided additional information regarding treatment persistence. No significant differences in rates of attrition were found among youth in treatment for suicidal ideations, history of abuse or neglect, or presence of attention-deficit symptoms (Dierker et al., 2001). In contrast to the adolescent research, the children and adolescents in Dierker et al.’s (2001) study who presented with depressed and isolated behaviors $(p<.01)$ were significantly more likely to leave treatment prematurely. Consistent with Baruch et al.’s (2009) research on adolescent clients, higher amounts of referral reasons $(p<.05)$ were found to be predicative of youth prematurely terminating treatment, as well as those with problems related to substance abuse $(p<.01$; Dierker et al., 2001).

Despite the clear findings found in adolescent research, findings regarding the predictive nature of aggression and other negative externalizing behaviors are conflicting in the research examining children and adolescents. Dierker et al. (2001) found homicidal ideations and aggressive/oppositional behaviors were not predictive of treatment completion. A subsequent study of 242 families of children aged 3-14 who were referred to an outpatient treatment program for aggressive and antisocial behaviors found 39.9\% of the participants terminated prematurely (Kazdin, et al., 1997). In contrast to Dierker et al.’s (2001) findings, Kazdin et al. 
(1997) found higher incidences of antisocial behaviors $(t=3.16, p<.01)$ were predictive of premature termination from counseling.

Environmental variables. In addition to individual factors, researchers have also examined environmental variables as they related to child and adolescent persistence in counseling. Venable and Thompson (1998) proposed that understanding familial characteristics might serve to help predict early termination among child and adolescent clients. Although demographic variables of the child or adolescent client were not indicative of premature termination in other studies, Kazdin et al. (1997) found that parents who terminated treatment for their children prematurely were more likely to identify as members of a non-dominant ethnic group, single, reported lower socioeconomic status (SES), and were younger than those who persisted in counseling.

Researchers have also explored the impact of parenting practices on treatment persistence. Parental intrapunitive hostility, characterized by "self-criticism and delusional guilt" was found to be predictive of early termination (Venable \& Thompson, 1998, p. 292). This may be explained by parental avoidance of self-reflection regarding their role in the manifestation of their children's presenting concerns. Parents who decided to prematurely leave treatment for their children were also more likely to have negative child-rearing practices $(t=8.30, p<.001)$ (Kazdin et al., 1997). Dierker et al.’s (2001) examination of familial characteristics revealed that several factors were either inconclusive or non-predictive of children and adolescents' persistence in counseling; these factors included: family size, insurance availability, and family pathology.

Beyond parental characteristics, Kazdin et al., (1997) asserted that parental interest in counseling predicted their children's length of treatment. Of the children that did not return to 
counseling beyond their intake session, 74\% of their parents expressed disinterest in the counseling process. Those who terminated prematurely reported significantly higher levels of barriers to treatment (Kazdin et al., 1997). These barriers included unique interactions between many factors, including parents’ perceived relationship with the counselor, relevance of treatment, treatment demands, and stressors-obstacles. Additionally, time spent on the waiting list prior to receiving treatment was associated with premature termination. For cases that were considered chronic due to their presenting problems, longer times spent on the waiting list further increased the likelihood that parents would terminate their children's services prematurely (Dierker et al., 2001). Thus, it is evident that parental factors and parents’ counseling experience play critical roles in understanding children and adolescents' continuation of counseling. Attrition in Child Counseling

In comparison to research examining attrition for adolescent clients, there is a greater depth of knowledge regarding attrition for child clients. Similar to adolescent attrition, Tsai and Ray (2011) noted that across studies, factors that contribute to premature termination for children are inconclusive. Due to children's reliance on family for continuance of treatment, familial factors have the ability to have a significant impact on the completion of treatment (Kazdin \& Mazurick, 1994).

Tsai and Ray (2011) examined archival data to explore rates of premature termination for children between the ages of 3 and 10 in a university-based community-counseling clinic. Of the 198 children who received individual play therapy, Tsai and Ray (2011) found that 63.6\% of the children terminated prior to a mutual agreement by the parent(s) and counselor. Additionally, the researchers reported reasons for termination noted in the files: $23.8 \%$ had three consecutive 
no-shows, $20.6 \%$ did not return to counseling after the college summer or winter break, $22.2 \%$ indicated not being interested in continuing treatment, $11.9 \%$ transferred services elsewhere, 7.1\% reported having a schedule conflict, $7.1 \%$ moved, and 7.1\% indicated another reason for discontinuing services. These findings highlight the role that parental decisions play in the continuance of children in counseling.

Personal variables. In addition to examining parental characteristics, researchers explored children's characteristics to identify variables that may be predictive of premature termination. Tsai and Ray (2011) examined several demographic variables: gender, age, ethnicity, and special education, none of which was statistically significant when comparing children who completed treatment and those who terminated prematurely. Tsai and Ray (2011) examined children's behavioral and emotional symptom severity and found that heightened severity of problems at intake lead to a higher incidence of treatment dropout. Kazdin and Mazurick (1994) also supported the finding that there was no relationship between the child's age and sex, but the severity of the child's social, conduct, and delinquent behaviors were predictive of early termination. Exploration of this relationship revealed premature termination may be a result of the impact of the child's distress on the family system as a whole. This may also be explained by the prolonged treatment required to achieve clinical improvement for severe problems.

Concurrent with results in adolescent attrition (Dierker et al., 2001), premature termination in children was found to be more likely among children with more severe delinquent behaviors, conduct disorder, and externalizing behaviors (Baruch et al., 1998; Kazdin, 1990). The impact of school and peer influence was also considered. For children with negative externalizing behaviors, treatment dropout was further increased for children with reported peers who also engaged antisocial behaviors (Kazdin \& Mazurick, 1994). Further, researchers reported 
that problems in school, academic delays, and lower IQ scores were related to premature termination (Baruch et al., 1998; Kazdin \& Mazurick, 1994). Linking personal and environmental variables, researchers have examined cultural differences among children who prematurely terminate from counseling and those who persist in counseling.

Cultural differences. Although Tsai and Ray (2011) found that ethnicity was not predictive of treatment completion, several researchers found children from non-dominant ethnic groups were more likely to terminate counseling prematurely (Kazdin et al.,1995; Kazdin \& Mazurick, 1994). An examination of families $(N=279)$ attending outpatient counseling for their child (ages 3-13) found that African American families (59.6\%) were more likely to terminate prematurely than White families (41.7\%; Kazdin, et al., 1995). As counseling progressed from week to week, African American families were 1.67 times more likely to leave counseling than White families, meaning that in addition to having higher rates of attrition, African American families were also more likely to have shorter duration of treatment. In some instances, characteristics predictive of attrition were different between racial groups. For White families, socioeconomic disadvantage, overall child symptoms, and family constellation characteristics (e.g., single parent families) were predictive of attrition. For African American families, the child's academic functioning was related to attrition. Some characteristics were similar for both racial groups, including child antisocial behavior, parental history of antisocial behavior, adverse child-rearing practices, and parental stress (Kazdin et al., 1995). Although the researchers found differences and similarities between racial groups, additional consideration should be given to the assumption that differences are solely attributed to race instead of environmental factors such as SES, or cultural differences with counselors, which may account for more between-group 
differences than race. It is imperative to understand the interaction effects of familial characteristics when examining children's persistence in counseling.

Environmental variables. Because children rely on their parents for both caretaking and treatment needs, familial characteristics are a focus for researchers examining children’s persistence in counseling. Findings are inconclusive for SES and parent relationship status. Although Tsai and Ray (2011) found SES and parent relationship status were not associated with continuity of treatment, several researchers have found socioeconomic disadvantage and single parent relationship status to be associated with premature termination in children (Kazdin, 1990; Kazdin \& Mazurick, 1994; Prinz \& Miller, 1994). Additionally, Kazdin and Mazurick (1994) found that children who resided in a home headed by young mothers, a non-biological parent, or a single parent were more likely to terminate counseling prematurely than those with intact two parent households. The presence of such characteristics may be associated with households that are characterized by increased parental stress.

Researchers have also examined how parental stress impacts children's persistence in counseling. Across studies, heightened levels of parental stress were positively correlated with premature termination from counseling (Kazdin \& Mazurick, 1994; Kazdin et al., 1995; Prinz \& Miller, 1994), specifically stress experienced by mothers (Kazdin, 1990). An examination of factors that contributed to familial stress revealed greater occurrence of life events, on the Parent Stress Index (PSI; Abidin, 1993). Congruent with the research on child and adolescent attrition, parents with adverse child rearing practices were significantly more likely to prematurely terminate their children's counseling compared to parents who did not report adverse child rearing practices (Kazdin \& Mazurick, 1994). 
Parental perception of counseling is also vital to ensuring that children persist in counseling. An examination of parents’ perceptions of treatment discovered parents of children who terminated early from treatment reported a belief that counseling was not producing the expected results (Attride-Stirling et al., 2004). Additionally, parents reported an overall sense of confusion about the counseling process. Although parents with children who persisted in counseling reported inconveniences around logistical aspects of counseling, those who terminated prematurely reported these inconveniences as being unmanageable. Thus, parents rated obstacles as having greater importance than the perceived growth of their children in counseling (Attride-Stirling et al., 2004). This is supported by Tsai and Ray’s (2011) finding that parents of children who completed treatment attended an average of 6.28 parent consultations in comparison to 3.45 parent consultations with parents who prematurely terminated, indicating a medium effect size (Cohen, 1992). Building on the findings from the child and adolescent literature, increased parental involvement in the counseling process serves as a protective factor against premature termination from counseling for children. Due to the complex interactions and inconclusive findings across variables, counselors should examine factors across all facets of their client's lives to fully understand their likelihood to persist in counseling. An examination of the developmental continuum leads to research on attrition in adult counseling. Attrition in Adult Counseling

Given the lack of knowledge regarding factors that contribute to adolescent treatment attrition, it may be beneficial for counselors to understand characteristics across the lifespan. Due to the autonomous nature of adulthood, researchers have focused on personal variables (i.e., client characteristics) to understand factors contributing to premature termination. Researchers examining attrition in the adult population have often focused on two treatment settings, 
university-based counseling centers, and programs for specialized concerns (e.g., post traumatic stress disorder, domestic violence).

Personal variables. Across studies, researchers have examined the impact of demographic variables and presenting concerns on adult clients' treatment persistence. Congruent with research across the lifespan, gender and number of presenting problems were not predictive of attrition (Lampropoulos, Schneider \& Spengler, 2009; Levy, Thompson-Leonardelli, Smith \& Coleman, 2005; Longo, Lent \& Brown, 1992). Other factors such as socioeconomic disadvantage, lower education levels, and identification with non-dominant populations were consistently associated with premature termination (Reis \& Brown, 1999; Wiezbicki \& Pekarik 1993).

Levy et al. (2005) reviewed archival data $(N=1,461)$ from a university-based counseling center. In their study, attrition was defined as those clients who did not return after their initial intake session. Consistent with the literature on childhood attrition, African American adult clients had higher rates of attrition (24.9\%) then White clients (18.9\%). As noted previously, these results should be further examined to consider other variables to ensure that assumptions are not made solely on a client's race.

Lampropoulos et al. (2009) examined rates of attrition at a university-based training clinic for clients aged 17-82 years. Of their sample, 16.1\% terminated counseling immediately following their intake session, 57.4\% terminated counseling prior to their counselor's recommendation, and only $26.6 \%$ completed treatment. Unique interactions between four variables were examined: age, income, perceived client difficulty, and the Global Assessment of Functioning (GAF) Scale. For clients over 40.5 years old, age was the most salient predictor of attrition, regardless of the other four factors. For clients under 40.5 years old, income below 
$\$ 20,000$ a year proved to be the best indication of termination. Factors that did not predict termination included education, history of treatment, employment status, referral source, number of children within the household, and urgency of presenting problems (Lampropoulos et al., 2009). Thus, there continues to be interaction effects among variables that contribute to a client prematurely terminating from counseling.

Beyond demographic variables, Longo et al. (1992) assessed problem severity, selfefficacy, expectations for counseling, motivation, and counselor experience to determine which variables best predicted premature termination. Of their sample $(N=139), 15 \%$ of the clients prematurely terminated, when defined as the client not returning after the initial intake session. The researchers conducted a discriminant analysis and found that above all other factors, selfefficacy $\left(\eta^{2}=.19\right)$ and motivation $\left(\eta^{2}=.10\right)$ were the most salient predictors in discerning perseverance in counseling. Both of these variables support the notion that internal, rather than external, motivation is an important factor in client persistence.

Researchers also focused on factors contributing to attrition in specialized adult treatment programs. Chang and Saunders (2002) reviewed archival data from a family service agency that provided a domestic violence offender program. Their sample consisted of 218 men, and had an overall 38\% ( $n=84)$ dropout rate which included men who did not return after the initial intake ( $n=40$ ), or did not complete the program $(n=44)$. A statistically significant relationship was found between older participants, higher levels of education, and higher levels of self-reported anger and treatment persistence. The researchers suggested these characteristics contributed to program continuation by clients' heightened awareness for negative consequences for their actions (Chang \& Saunders, 2002). 
Matthieu and Ivanoff (2006) completed a comprehensive review of ten years of adult PTSD treatment outcomes. From these studies $(N=11)$, they found that clients who terminated treatment prematurely had more severe PTSD symptomology at intake, reported history of psychological treatment, had lower incomes, and were more likely to be unemployed than those who persisted in counseling. While these results were concluded from specialized treatment modalities, they provide valuable evidence into the limited knowledge known about attrition in adult counseling.

Environmental variables. Researchers also explored characteristics of the counselor client relationship to understand variables that may be associated with clients’ persistence in counseling. Long et al. (1992) found that depth of counselor clinical experience was not related to clients' persistence in counseling. This finding was not supported by Lampropoulos et al.'s (2009) research on rates of attrition in a university-based training clinic. Lampropoulos et al. (2009) proposed that consideration should be given to the typical discrepancy in age between the counselors and the client, with counselors in training clinics often lower in age than the clients they are serving, rather than attributing the association solely to a counselor's experience. This finding may contribute to high rates of termination among older adult clients in training clinic settings.

As previously noted, the therapeutic alliance may serve as a protective factor against premature termination. Reis and Brown (1999) proposed that counselors' inclusion of a professional disclosure statement, which includes information on counseling expectations, may increase therapeutic alliance. Converging the expectations of client and counselor helps in stabilizing the therapeutic relationship and may reduce the likelihood of unplanned termination. Lack of contact with counselors has also been proposed as a variable predictive of attrition. Long 
at al. (1992) found increased time spent on a waiting list was associated with increased rates of attrition, with the impact significantly increasing after three weeks. It was suggested that increased time spent on the waiting list may be perceived by the clients as feeling unsupported by the clinical staff, thus decreasing their motivation to engage in counseling (Levy et al., 2005).

Researchers examining characteristics related to adult premature termination have explored a number of personal and environmental variables. From these studies, it became clear that clients' desire to engage in treatment, although very different for many clients, impacted their ability to remain in counseling. Beyond this, many findings examining specific characteristics conflict or misalign with what has been found across the lifespan, leaving counselors with an incomplete understanding of characteristics that are predictive of client premature termination.

Attrition Across the Lifespan

Throughout the lifespan, there remains a lack of clarity regarding what factors contribute premature termination among clients. In part, this is due to the lack of standardization of defining what constitutes premature termination. A review of the literature suggests that clients who are motivated for treatment are more likely to persist in counseling. Across studies and populations, client motivation was identified in many different ways, including self-reported feelings of anxiety, depression, and anger, or being self-referred for counseling (Baruch et al., 2009; Chang \& Saunders, 2002; Longo et al., 1992; Pagin et al., 2004; Sunseri 2003). Additionally, it is evident that for youth, parental motivation for treatment is essential for continuance of treatment (Attride-Stirling et al., 2004; Kazdin et al., 1997; Tsai \& Ray, 2011). Clients who engaged in counseling due to environmental factors, either referred by others or attending counseling due to externalizing behaviors (e.g., delinquency and substance abuse), 
often dropped out of counseling prematurely (Baruch et al., 2009; Pagin et al., 2004; Sunseri 2003).

Conclusion

Adolescence continues to be a stage of development that is understudied and often misunderstood (Ausubel, 2002). Due to the lack of current research in the area of adolescent attrition, understanding factors across the lifespan may serve as a framework for further exploration of adolescent treatment outcomes. Frequently, assumptions are made based on an adolescent's status as being post-childhood and pre-adulthood, without understanding the unique characteristics that distinguish this point of development (Ausubel, 2002). Researchers have examined both personal and environmental factors to create a framework for gaining a holistic understanding of factors that are predictive of an adolescent persisting in counseling. The current study aims to add to the established framework, which includes an understanding of the predictive power of presenting problems, both internal (e.g., depression, anxiety) and external, (e.g., delinquent behaviors, conduct problems) in relation to persistence in counseling (Baruch et al., 2009; Pagnin, et al., 2004). Researchers identified familial characteristics, particularly stress, as significant factors impacting client’s persistence in counseling (Kazdin \& Mazurick, 1994; Kazdin et al., 1995; Prinz \& Miller, 1994). Further exploration of parental stress and the parentchild relationship will aid in understanding the impact of familial characteristics on treatment persistence. Examination of time spent on the waiting list as well as adolescent involvement in the intake process will provide further insight into the impact that an early establishment of a therapeutic alliance has on an adolescent's persistence in counseling (Blotcky \& Friedman, 1984). 
APPENDIX C

\section{EXTENDED METHODOLOGY}


The purpose of the current study was to develop a model of persistence in individual adolescent counseling. This was achieved through conducting a series of univariate and multivariate analyses with archival data obtained from client files from a university-based counseling clinic. A multiple linear regression was conducted to examine predictors of number of counseling sessions attended, followed by a predictive discriminant analysis (PDA) to examine the predictability of the model. This chapter includes the research questions to be addressed, participant information, research procedures, data analysis, and limitations. Research Questions

Based on conclusions drawn from previously reviewed research, the current study is designed to examine the following research questions:

1. How much of the variance in the number of counseling sessions attended by adolescent clients is explained by adolescent involvement in intake, time spent on wait list, levels of parental stress, and parent perceptions of adolescent internalizing and externalizing behaviors?

2. a. What is the demographic and clinical profile of adolescent clients who prematurely terminated from counseling compared to those who persisted in counseling?

b. To what degree can gender, age, whether an adolescent is involved in intake, time spent on wait list, levels of parental stress, and parent perceptions of adolescent internalizing and externalizing behaviors predict whether an adolescent will terminate prematurely or persist in counseling? 


\section{Definition of Terms}

Counseling is defined as “a professional relationship that empowers diverse individuals, families, and groups to accomplish mental health, wellness, education, and career goals” (American Counseling Association, 2010).

Adolescents are defined as clients who, at intake, were between the ages of 12 and 18 and living with caretakers.

Premature termination is defined as a not completing at least two counseling sessions. This was established by a review of previous literature on attrition and limits the external effects of the therapeutic experience.

Persistence is defined as attending at least eight counseling sessions. In a meta-analysis, Lambert (2013) found most clients show clinically significant improvement after seven sessions, and resolution of presenting problems occurs after eight sessions. Thus, it was determined that clients who attended at least eight sessions met the criteria for persisting in counseling.

Parental stress is measured by four subscales:

Adolescent-related stress is measured by the adolescent domain (AD) on the Stress Index for Parents of Adolescents (Sheras, Abidin \& Konold, 1998). The AD measures the levels of stress experienced by parents or guardians as a result of characteristics of adolescent clients. These characteristics include adolescents' mood and behaviors.

Parent-related stress is measured by the parent domain (PD) on the Stress Index for Parents of Adolescents (Sheras et al., 1998). The PD measures the level of stress experienced by parents or guardians as a result of parenting on other facets of their life (e.g., relationships, parenting competency). 
Parent-client relationship is measured by the adolescent-parent relationship domain (APRD) on the Stress Index for Parents of Adolescents (Sheras et al., 1998). The APRD measures the parent's perceived quality of the relationship between parents or guardians and adolescents.

Life stressors is measured by the life stressors domain (LS) on the Stress Index for Parents of Adolescents (Sheras et al., 1998). The LS measures the occurrence of stressful life events within the family system.

Parent Perceptions of Adolescent Internalizing and Externalizing Behaviors is measured by the Internalizing and Externalizing subscale scores on the Child Behavior Checklist (Achenbach \& Rescorla, 2001). Examples of internalizing behaviors include: “Cries a lot” and "Rather be alone.” Examples of externalizing behaviors include: "Harms self,” "Cruel to Animals.”

Time spent on wait list is defined as the number of days between the intake session and the point of contact from a counselor to schedule the first counseling session.

Adolescent involvement in the intake is indicated by whether an adolescent was involved in the intake session or if parent or guardian attended the session independently. Participants

Archival data were acquired from clinical files $(N=74)$ of adolescent clients who sought services at a university-based community-counseling clinic between 2007 and 2014. The clinic served as a training facility for master's and doctoral students at an accredited counselor education program in the southwestern United States. The clinic provided services to approximately 150 clients per week and served clients ages 3 through adulthood. An array of treatment modalities were available, including individual, group, couple, and family counseling; 
services were provided based on a sliding scale fee structure. The majority of the counselors were advanced doctoral students. The master's level counselors were completing their internship. All counselors engaged in weekly triadic and group supervision.

Selection of participants was based on several inclusion criteria. Firstly, clients were between the ages of 12 and 18 at the time of intake. The participants must have also been living with caretakers and have completed an intake session requesting individual counseling services. Additionally, the required assessments, the Child and Adolescent Background Information Form (CABIF), an Intake Summary Report, a Child Behavior Checklist (CBCL; Achenbach \& Rescorla, 2001), and a Stress Index for Parents of Adolescents (SIPA; Sheras et al., 1998), must have been completed within the file.

Demographic Information

Of the adolescent files examined $(N=74), 89.2 \%(n=66)$ were closed-client files and $10.2 \%(n=8)$ were clients currently receiving services. The sample was $61.1 \%(n=44)$ female and 38.9\% ( $n=28)$ male, with two participants not reporting their gender. The mean age of the sample was $14.23(S D=1.65)$ and the mean academic grade in school was $8.44(S D=2.30)$. The participants identified the following ethnicities: White (67.6\%, $n=50)$, Hispanic/Latino(a) (14.9\%, $n=11)$, Bi-racial (10.8\%, $n=8)$, African American $(4.1 \%, n=3)$, and Asian (2.7\%, $n=$ 2). An examination of family household income revealed: $\$ 40,001+(39.7 \%, n=23)$, less than $\$ 15,000(20.7 \%, n=12), \$ 20,001-\$ 30,000(15.5 \%, n=9), \$ 15,001-\$ 20,000(12.1 \%, n=7)$, $\$ 30,001-\$ 40,000(12.1 \%, n=7)$. Sixteen (21.6\%) participants did not report their household income. See Table C.1 for a summary of descriptive statistics. 
Table C.1

Demographic Descriptive Statistics

\begin{tabular}{lcc}
\hline & \multicolumn{2}{c}{$(\boldsymbol{N}=\mathbf{7 4})$} \\
\cline { 2 - 3 } Demographic Variable & $\boldsymbol{M}$ & $\boldsymbol{S D}$ \\
\hline Age & 14.23 & 1.65 \\
Grade & 8.44 & 2.30 \\
& & \\
\hline Gender & $\boldsymbol{N}$ & $\mathbf{\%}$ \\
$\quad$ Female & 44 & 61.1 \\
$\quad$ Male & 28 & 38.9 \\
Race/ethnicity & & \\
$\quad$ African American & 3 & 4.1 \\
$\quad$ Asian & 2 & 2.7 \\
Bi-racial & 8 & 10.8 \\
Hispanic/Latino(a) & 11 & 14.9 \\
White & 50 & 67.6 \\
& & \\
SES & & \\
$\quad$ <15,000 & 12 & 20.7 \\
\$15,001- \$20,000 & 7 & 12.1 \\
\$20,001-\$30,000 & 9 & 15.5 \\
\$30,001-\$40,000 & 7 & 12.1 \\
> \$40,001 & 23 & 39.7 \\
& & \\
\hline
\end{tabular}

Procedures

I obtained permission from the clinical director to create a database of the adolescent client files and analyze the data for research purposes. I obtained approval from the University Institutional Review Board (IRB \#14451) prior to analyzing the data. Due to the utilization of archival data, clinic procedures were already established. Parents or guardians interested in services for their adolescents attended an intake session at the clinic. Upon arrival, they completed paperwork that included a CABIF, Intake Summary Report, CBCL, and SIPA. They then completed an intake session with an assigned counselor to complete the informed consent process. The counselor provided information on the nature of the training clinic and data 
collection procedures. The guardians provided consent for the information gathered to be used for education and research purposes. Upon completion, clients were put on a waiting list until being scheduled by a counselor for weekly 50-minute sessions.

To assist in data entry, and reduce entry error, I generated a codebook to indicate the respective established codes (Appendix D). I created a SPSS database containing all pertinent file information. To protect client confidentiality, data entry took place at the counseling clinics, on a password-protected computer. I assigned a three digit identification number to each file, which was stored in a separate, password-protected excel file. The database included all available clinical information, exceeding the variables examined in the current study to allow for future analyses. Identifying information such as name, address, social security number, or family members’ names were not included in the database.

Instrumentation

Child and Adolescent Background Information Form (CABIF). The clinical director at the university-based training clinic developed the CABIF to gather biopsychosocial information on clients and their immediate family. The CABIF was also utilized to collect information regarding the client's presenting concerns and familial stressors. The current study examined the following demographic variables: age, gender, grade, ethnicity, and SES. See Appendix F for a copy of the CABIF.

Intake Summary Report. The clinical director also developed the intake summary report to document the details of the intake session. For the current study, I examined the form to determine the number of days between intake and the first scheduled counseling session, which represented the amount of days spent on waitlist. The document was also examined to determine 
whether the adolescent was involved in the intake session, or if the guardian(s) completed the process independently. See Appendix F for a copy of the Intake Summary Report.

Child Behavior Checklist 6-18 (CBCL; Achenbach \& Rescorla, 2001). The CBCL is an instrument that measures adolescents' behavioral and emotional problems. The CBCL is completed by parents or guardians and designed for youth aged 6-18. Parents are asked to indicate the occurrence of behaviors within the past six months. Results are reported according to three behavioral domains: internalizing, externalizing, and total behavior. According to Achenbach and Rescorla (2001), externalizing behaviors are defined as the outward expression of internal distress (e.g., aggression, delinquency). In contrast, internalizing behaviors are defined as the inward expression of internal distress (e.g., depression, anxiety). Parents respond to 13 questions regarding their child's social interactions, school experience, and activity involvement to capture a holistic view of the child from the respondent's perspective. The parents then respond to 113 Likert-scale questions to capture their children's behavior during the previous six months. Scaling of questions ranges from: " $0=$ not true", " 1 = somewhat or sometimes true", to " 2 = very true or often true." Scores are reported based on percentiles and normalized $T$ scores, which are standardized scores that indicate a child's functioning based on comparative analysis of other children of the same sex and age. Researchers are able to examine normative data to determine if children's scores fall within the clinical or non-clinical range.

Due to the utilization of archival data, internal consistency of the CBCL with the current sample was not accessible. Despite this, Achenbach and Rescorla (2001) reported the use of the CBCL across several studies during the past 20 years, establishing strong validity for the assessment. Content and criterion-related validity were established, finding that across all items, the CBCL significantly discriminated $(p<.01)$ between demographically comparable referred 
and non-referred children. The CBCL was found to have high reliability, with a Cronbach's alpha of .90 to .97 for the three subscales. Inter-interviewer reliability based on an administration to 241 children ranged from .93 to .96 , indicating high inter-interviewing reliability. Test-retest reliability was also found to be strong, ranging from .95 to 1.00 . Test-retest reliability for the behavior problem items was calculated using the intraclass correlation coefficient from one-way analyses of variance, resulting in $.95(p<.01)$. These results demonstrate that the CBCL has high reliability and validity.

For purposes of the current study, to capture parent perceptions of adolescent mental health concerns, I examined $T$-scores from the internalizing and externalizing subscales. Sample questions for internalizing behaviors include: “Cries a lot," "Rather be alone”; and externalizing behaviors: "Harms self," "Cruel to animals.” High $T$-scores on the scales indicate clinically significant deviance from the behavior to be expected for that gender and age range. Normative scores are reported, and clinical significance is indicated for scores above the $98^{\text {th }}$ percentile. Scores within the $93^{\text {rd }}$ to $97^{\text {th }}$ percentile are deemed as having borderline clinical significance, and those below the $93^{\text {rd }}$ percentile are non-clinical.

Stress Index for Parents of Adolescents (SIPA; Sheras et al., 1998). The SIPA is a 112item instrument that measures stress related to a caretaker's interactions with an identified adolescent between the ages of 11 and 19. The SIPA is an adaptation of the Parenting Stress Index and is designed to capture the changes in parents' process as their children move into adolescence (Abidin, 1993). The assessment examines stress on a five point Likert scale ranging from: " $1=$ Strongly Agree" to " 5 = Strongly Disagree." The questions assess stress across four domains: Parent-Domain, Adolescent-Domain, Adolescent-Parent Relationship, and Life Stressors. The clinical values for the four domains are presented in Table C.2. 
Table C.2

SIPA Clinical Scores

\begin{tabular}{lccc}
\hline & $\begin{array}{l}\text { Borderline } \\
\text { Clinically } \\
\text { Significant }\end{array}$ & $\begin{array}{l}\text { Clinically } \\
\text { Significant }\end{array}$ & Clinically Severe \\
\hline $\begin{array}{l}\text { Adolescent-Focused } \\
\text { Stress }\end{array}$ & $112-120$ & $121-131$ & $132-200$ \\
Parent-Focused Stress & $95-100$ & $101-109$ & $110-170$ \\
Adolescent-Parent & $43-46$ & $47-53$ & $54-80$ \\
Relationship & & 5 & $6-22$ \\
Life Stressors & 4 & 5 & \\
\hline
\end{tabular}

The SIPA is an empirically validated assessment to measure degree of stress experienced by caretakers of adolescents. The system utilized in the current study did not allow access to internal consistency scores for this sample. Despite this, Sheras et al. (1998) established a high degree of reliability for the SIPA. The SIPA demonstrated a moderate to high degree of internal reliability, with alpha coefficients ranging from .81-.97 across all scales and subscales (Sheras et al., 1998). Test-retest reliability coefficients were high, ranging from .74-.93, across a four-week interval. Convergent and discriminant validity of the instrument is demonstrated by higher scores for guardians of adolescents in a clinical sample compared to those in a normative sample Construct validity was supported by a factor analysis, which demonstrated empirical differences across the domains. The high validity indicates the SIPA can effectively assess parents struggling with stress related to parenting their adolescents as well as identifying an adolescent's diagnosis based on DSM-IV-TR (APA, 2000) criteria.

To understand levels of parental stress, all four domains (parent-domain, adolescentdomain, adolescent-parent relationship, and life stressors) were examined. The parent-domain "measures the level of stress experienced by a parent as a function of the effect on parenting on other life roles, the relationship with a spouse or partner, social isolation, and parenting 
competence” (Sheras et al., 1998, p. 6). The parent-domain contains 35 likert-scale questions and assesses parent stress on four subscales: life restrictions, relationship with spouse/partner, social alienation, and incompetence/guilt. The adolescent-domain "measures the level of stress experienced by a parent as a function of the characteristics of his or her adolescent” (Sheras et al., 1998, p. 6). The adolescent-domain contains 40 likert-scale questions and assesses adolescent attributes across four subscales: moodiness/emotional liability, social isolation/withdrawal, delinquency/antisocial, and failure to achieve or persevere. The adolescent-parent relationship domain "measures the perceived quality of the relationship that the parent has with the adolescent, such as the degree of communication and affection between them” (Sheras et al., 1998, p. 6). The adolescent-parent relationship domain is a 16 likert-question scale that assesses the quality of the relationship as experienced by the caretaker through questions measuring the level of communication, self-perceived confidence in their parental role, and the quality of their interactions. The life stressors domain provides an opportunity to "indicate the number of stressful evens that the parent has experienced in the past year” (Sheras et al., 1998, p. 6). This may include loss of a job, death of a loved one, or illness. Combining scores across all four subscales can also attain a cumulative score, total stress. Due to the cumulative nature of the total stress scale, it was not examined in the current study. See Table C.3 for a summary of the measures utilized from the SIPA. 
Table C.3

SIPA (Sheras et al., 1998)

\begin{tabular}{|c|c|c|}
\hline Scale & $\begin{array}{c}\text { Items } \\
(N=112)\end{array}$ & Definition \\
\hline Parent-Domain & 35 & $\begin{array}{l}\text { Measures the level of stress experienced as a } \\
\text { function of their role as a parent of an adolescent. }\end{array}$ \\
\hline Adolescent-Domain & 40 & $\begin{array}{l}\text { Measures the level of stress experienced by a parent } \\
\text { as a function of the characteristics of his or her } \\
\text { adolescent. }\end{array}$ \\
\hline $\begin{array}{l}\text { Adolescent-Parent } \\
\text { Relationship }\end{array}$ & 16 & $\begin{array}{l}\text { Measures the perceived quality of the relationship } \\
\text { that the parent has with the adolescent, such as the } \\
\text { degree of communication and affection between } \\
\text { them. }\end{array}$ \\
\hline Life Stressors & & $\begin{array}{l}\text { Provides an opportunity for parents to indicate the } \\
\text { number of stressful evens they have experienced in } \\
\text { the past year. }\end{array}$ \\
\hline
\end{tabular}

Data Analysis

All data were entered and analyzed in SPSS version 22. Participants’ demographic information was analyzed, and descriptive statistics are reported to gain an understanding of the profile of clients who presented to the clinic. I analyzed frequencies for gender, ethnicity, and SES, as well as the mean and standard deviation for age and grade of the participants.

Research Question 1. I conducted a linear multiple regression to understand how much of the variance in the number of counseling sessions attended was explained by adolescent involvement in the intake, the time spent on the waitlist, internalizing and externalizing behaviors, and four domains of parent-reported stress. Due to the dependent variable (number of sessions attended) being continuous in nature, only closed client files ( $n=66)$ were included in this analysis. Using G*Power 3.1 (Faul, Erdfelder, Buchner \& Lang, 2009), I conducted an a 
priori test to determine the necessary sample size. A sample size of 43 was needed for a moderate effect size, $\left(f^{2}=.15\right)$, power $=.80, \alpha=.05$, for the eight predictor variables examined. Thus, the available sample exceeded the required sample size.

I utilized standard entry to simultaneously examine the predictor variables (Field, 2009). Standard entry is the most commonly utilized method of variable entry and reduces the likelihood of interpretation error that is often present in step-wise entry (Huberty \& Olejnik, 2006). The first step of the data screening process included detection of missing variables. Of the analyzed cases ( $n=66)$, two cases were missing scores on a single variable. The missing scores, which were present on the $L S$ subscale of the SIPA, were replaced with the mean score for the sample. This method of supported by Field (2009) as an appropriate handling of missing data. After ensuring all cases had scores for each variable, preliminary analyses were conducted to ensure the data met the necessary assumptions for a multiple linear aggression.

Assumptions. Prior to running the multiple linear regression, I screened data to ensure the appropriateness of the analysis:

1. All variables must be either interval or categorical in nature, and the dependent variable must be continuous in nature (Field, 2009). Seven of the eight variables were interval, and one (adolescent involvement in the intake) was categorical. In addition, all variables had variance in their values (Field, 2009).

2. In multiple linear regression, perfect multicollinearity should not exist (Field, 2009). A correlational matrix revealed statistically significant correlations among the predictor variables, but no perfect linear relationships. 
3. Linearity should exist between the variables. A scatterplot illustrating the relationship between the continuous variables and number of sessions attended confirmed the data met the assumption of linearity (Pallant, 2013).

4. All continuous variables should be normally distributed (Tabachnick \& Fidell, 2001). To ensure normality, I examined the levels of skewness and kurtosis for each of the variables. This confirmed that the continuous variables fell reasonably within the parameters of normal distribution. Time spent on the waitlist was the only variable that had a skew score (1.23) that was outside of the parameters of \pm 1 ; because of this, no transformations to the data were deemed necessary.

5. There should be homoscedasticity among the variables. An inspection of the normal probability plot of the regression standardized residuals revealed the presence of mild heteroscedasticity. Because the data met all other assumptions, no data transformations were utilized.

Research Question 2. A series of univariate and multivariate analyses were conducted to examine the demographic and clinical differences between adolescents who prematurely terminated from counseling and those who persisted. A PDA was then conducted to determine to what degree the variables can predict whether an adolescent terminated prematurely or persisted in counseling. The goal of PDA is for a researcher to be able to accurately predict group membership based on a set of predictor variables (Sherry, 2006). The grouping variable was identified as adolescents' completion of treatment: those who prematurely terminated from counseling and those who persisted in counseling. Predictor variables were those that the researcher deemed important to the exploration of group difference. In PDA, it is recommended that the predictor variables be continuous in nature (Klecka, 1980). All variables met this 
criterion except for gender and whether the adolescent was involved in the intake, which I dummy-coded for analysis. For the PDA analysis, I also utilized standard entry to simultaneously examine the predictor variables (Field, 2009).

Assumptions. Prior to running the PDA, I screened data to ensure the appropriateness of the analysis. Several assumptions must be met prior to conducting a PDA:

1. There must be two or more naturally occurring groups (Tabachnick \& Fidell, 2001). In addition, groups must be exclusive and exhaustive across all cases; every case must be a member of a single group (Klecka, 1980). In the current study, premature termination was defined as clients who do not return to counseling either after the initial intake session or the first counseling session. Conversely, persistence was defined as clients who attended at least eight counseling sessions. A review of the literature, as well as an examination of the sample, supported the definition of the two groups. Researchers found that at least half of clients show clinically significant improvement after seven to eight counseling sessions (Lambert, 2013; Kadera et al., 1996). Additionally, researchers found high rates of termination within the first two counseling sessions (Hatchett \& Park, 2003). An examination of the frequency of sessions attended by the sample also supported the split in groups. Examination of the data indicated there was a sizeable group of clients who terminated prior to the second session $(n=35)$, very few clients who attended from two to seven sessions $(n=13)$, and a sizeable number who persisted at least eight sessions $(n=24)$. Consequently, preliminary analysis indicated there was a natural split amongst the groups.

2. There must be at least two cases per group, with a recommended minimum of three cases per group, per predictor variable. (Huberty \& Olejnik, 2006, Tabachnick \& Fidell, 2001). 
This data requirement also supports the exclusion of the adolescents who attended two to seven sessions in the model. For best practice, Poulsen and French (2008) suggested there should be four to five times the number of total cases for each predictor variables. In order to meet the requirements of the current research questions, 10 predictor variables, a minimum of 50 files must be analyzed.

3. The predictor variables should be normal at the univariate and multivariate levels (Tabachnick \& Fidell, 2001). To ensure normality, I examined the levels of skewness and kurtosis for each of the predictor variables. Only one predictor variable, Time on waitlist, exceeded the normal range with a slightly elevated skewness value of 1.23 . Two groups, Adolescent involvement in the intake and Life stressors, had values outside of the expected -1 or +1 , with values at -1.12 and -1.02 respectively. To further assess the need for data transformations, histograms for the identified variables were examined. An examination of the histogram plots revealed no significant need to perform transformations to the data. Mahalanobis $\left(\mathrm{D}^{2}\right)$ distance for the data was examined to determine multivariate normality (Henson, 1999). $\mathrm{D}^{2}=24.44$ does not exceed the critical value of 26.13, indicating there were not multivariate outliers within the dataset (Pallant, 2010).

4. DA is sensitive to the presence of outliers (Tabachnick \& Fidell, 2001). I screened for outliers on the univariate and multivariate level and made decisions on a case-by-case basis based on clinical judgment of the characteristics of the predictor variables. The origin of the outlier, whether due to entry error or population characteristics, was taken into consideration when determining the method for handling the case (Poulsen \& French, 2008). I ensured that all data, which were identified by SPSS as an outlier, were 
entered correctly. Due to the nature of the predictors, with scores derived from the true clinical experience of the subjects, all scores remained intact.

5. Poulsen and French (2008) explained that PDA is sensitive to homogeneity of variancecovariance matrices. To assess this, I examined the Box's $M$ statistic, $M=74.442 p=$ .004. According to Sherry (2006), a nonsignificant Box’s M ( $>$.001) indicates the data meets the assumption of homogeneity of variance. Meeting this assumption means that the covariances were equal across groups, and that the cases were derived from the same population.

6. PDA must have linearity among all sets of predictor variables within each group (Tabachnick \& Fidell, 2001). A scatterplot illustrating the relationship between the predictor variables for both groups confirmed the data met the assumption of linearity (Pallant, 2013). 
APPENDIX D

RESULTS 
The following presents the results from the examination of the research questions. To address the research questions, this section includes the results from the linear multiple regression as well as the PDA. To further examine the differences among adolescents who prematurely terminated versus those that persisted, results from univariate and multivariate analyses are also presented.

\section{Research Question 1}

A linear multiple linear regression was conducted to examine the first research question: How much of the variance in the number of counseling sessions attended by adolescent clients is explained by adolescent involvement in intake, time spent on wait list, levels of parental stress, and parent perceptions of adolescent internalizing and externalizing behaviors?

To meet the needs of the current research question, only closed-client files were included in this analysis $(n=64)$. The sample was 57.6\% $(n=38)$ female and 39.4\% $(n=26)$ male. The mean age of the sample was $14.39(S D=1.60)$, and the mean academic grade in school was 8.57 $(S D=2.32)$. The participants identified the following ethnicities: White $(71.2 \%)$, Hispanic/Latino(a) (13.6\%), Bi-racial (10.2\%), African American (3.4\%), and Asian (1.7\%). An examination of family household income revealed: $\$ 40,001+(36.7 \%)$, less than $\$ 15,000$ (20.4\%), \$20,001-\$30,000 (18.4\%), \$15,001-\$20,000 (12.2\%), \$30,001-\$40,000 (12.2\%). See Table D.4 for a summary of descriptive statistics. 
Table D. 4

Demographic Descriptive Statistics

\begin{tabular}{lcc}
\hline & \multicolumn{2}{c}{$(\mathbf{n}=\mathbf{6 6})$} \\
\cline { 2 - 3 } Demographic Variable & $\boldsymbol{M}$ & $\boldsymbol{S D}$ \\
\hline Age & 14.39 & 1.60 \\
Grade & 8.57 & 2.32 \\
& & \\
\hline Gender & $\boldsymbol{n}$ & $\mathbf{\%}$ \\
$\quad$ Female & 38 & 57.6 \\
Male & 26 & 39.4 \\
Race/ethnicity & & \\
$\quad$ African American & 3 & 4.9 \\
Asian & 1 & 1.5 \\
Bi-racial & 5 & 7.6 \\
Hispanic/Latino(a) & 10 & 15.2 \\
White & 47 & 71.2 \\
& & \\
SES & & \\
$\quad$ <15,000 & 11 & 21.6 \\
\$15,001- \$20,000 & 7 & 13.7 \\
\$20,001-\$30,000 & 8 & 15.7 \\
\$30,001-\$40,000 & 6 & 11.8 \\
$>$ \$40,001 & 19 & 37.3 \\
\hline
\end{tabular}

As indicated in Table C.5, the model was statistically significant $(F(8,57)=2.930, p=$ $.008)$ and accounted for $29.1 \%\left(R^{2}\right.$ adj $\left.=.192\right)$ of the variance in sessions attended. According to Cohen (1992) this model had a large effect size. The significant shrinkage examined may be explained by either sampling error of the presence of collinearity among several of the predictor variables examined. A correlational matrix revealed several statistically significant correlations between the predictor variables, indicating multicollinearity (See Table D.6). Because of this, squared structure coefficients were analyzed to determine the percentage of variance explained by each of the predictor variables (Kraha, Turner, Nimon, Zientek, \& Hensen, 2012; Thompson, 1992). 
Table D.7 displays the standardized regression coefficients ( $\beta$ ), the squared structure coefficients $\left(r_{\mathrm{s}}{ }^{2}\right)$, and the bivariate correlations for all predictor variables. Although the standardized regression coefficient for externalizing behaviors was not statistically significant ( $\beta$ $=-.205, t=-.954, p=.344$ ) an examination of the squared structure coefficients revealed the presence of suppressor effects within the model (Kraha, Turner, Nimon, Zientek, \& Hensen, 2012). Externalizing behaviors accounted for $43.82 \%$ of the variance explained in the number of sessions attended. Whether the adolescent was involved in the intake (29.16\%), internalizing behaviors (12.96\%), and parent-focused stress (10.30\%) also contributed to the variance explained in number of sessions attended. The remainder of the variables minimally contributed to the model: life stressors (3.9\%), adolescent-parent relationship (0.29\%), adolescent-focused stress $(0.22 \%)$, and time spent on the waitlist $(0.12 \%)$.

An examination of correlations revealed both positive and negative relationships between the variables and the number of sessions attended. Whether the adolescent was involved in the intake, internalizing behaviors, adolescent-parent relationship, and life stressors were all positively correlated with the number of sessions attended. Conversely, the number of days spent on the waitlist, externalizing behaviors, adolescent-focused stress, and parent-focused stress were negatively correlated with the number of sessions attended by the adolescent. Table D.5

Regression Summary Table

\begin{tabular}{lrrrcccc}
\hline Model & \multicolumn{1}{c}{ SOS } & $\boldsymbol{d} \boldsymbol{f}$ & Mean Square & $\mathbf{F}$ & $\mathbf{P}$ & $\mathbf{R}^{\mathbf{2}}$ & $\mathbf{R}^{\mathbf{2}}$ adj \\
\hline Regression & 2986.557 & 8 & 373.320 & 2.930 & $.008^{*}$ & .291 & .192 \\
Residual & 7262.973 & 57 & 127.421 & & & & \\
Total & 10249.530 & 65 & & & & & \\
\hline
\end{tabular}

Note. SOS: Sum of squares df: Degrees of freedom $\quad \mathrm{F}$ :F-statistic $\quad \mathrm{p}$ : $\mathrm{p}$-value $\mathrm{R}^{2}$ :Multiple $\mathrm{R} \quad \mathrm{R}^{2}$ adj: Multiple $\mathrm{R}$ adjusted *statistically significant at $\mathrm{p}<.01$ 
Table D.6

Pearson Correlation Coefficients

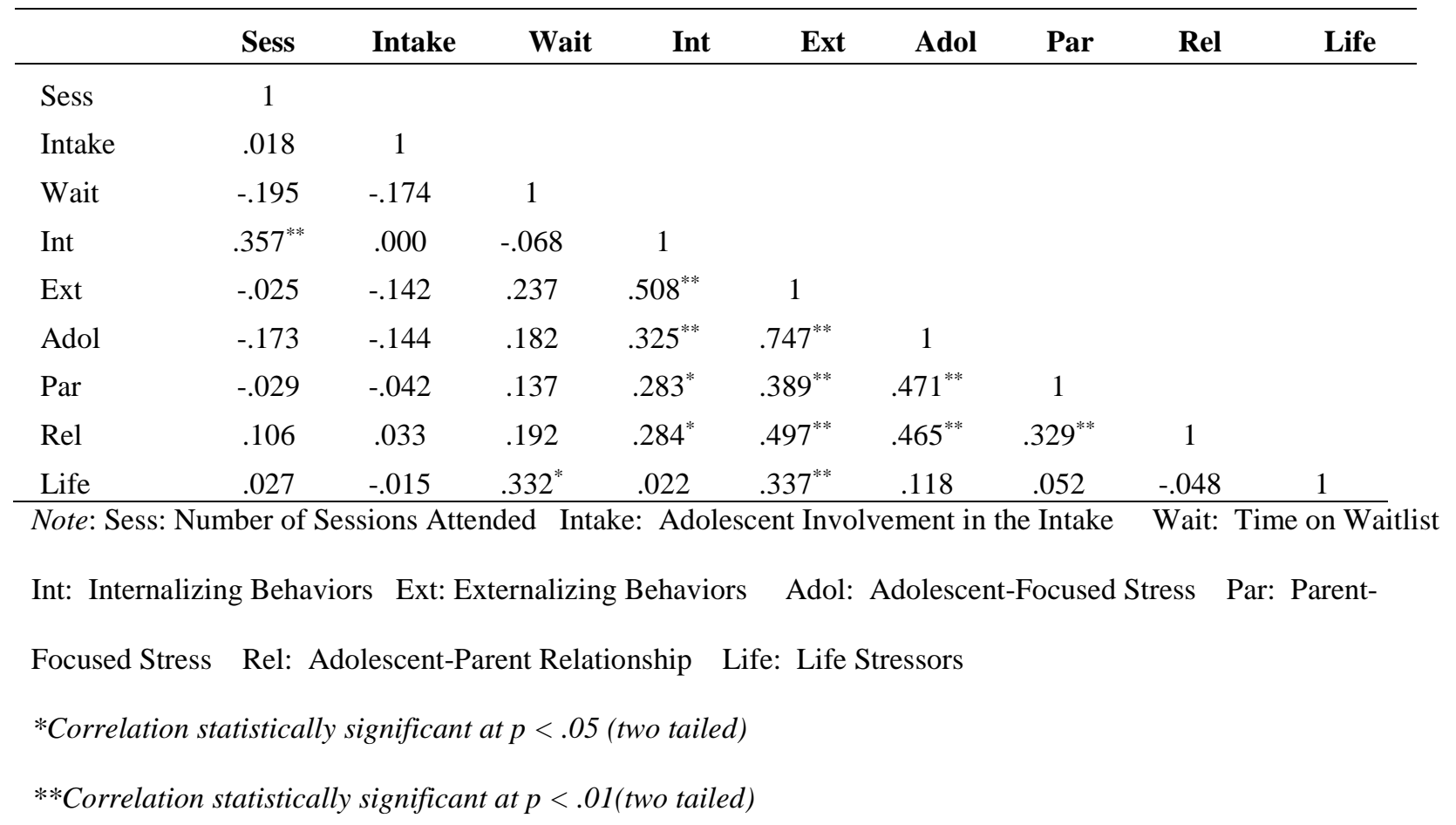

Table D.7

Beta Weights and Structure Coefficients for the Regression Model $(n=66)$ '

\begin{tabular}{|c|c|c|c|c|c|}
\hline Variables & $\boldsymbol{\beta}$ & $t$ & $p$ & $\mathbf{r s}^{2}$ & $\mathbf{r}$ \\
\hline Involvement in Intake & -.092 & -.791 & .432 & .292 & .018 \\
\hline Time on Waitlist & -.190 & -1.515 & .135 & .001 & -.195 \\
\hline Internalizing Behaviors & -.468 & 3.422 & .001 & .129 & .357 \\
\hline Externalizing Behaviors & -.205 & -.954 & .344 & .438 & -.025 \\
\hline Adolescent-Focused Stress & -.286 & -1.569 & .122 & .002 & -.173 \\
\hline Parent-Focused Stress & -.021 & -1.65 & .869 & .103 & -.029 \\
\hline Adolescent-Parent & .264 & 1.903 & .062 & .003 & .106 \\
\hline \multicolumn{6}{|l|}{ Relationship Stress } \\
\hline Life Stressors & .195 & 1.456 & .151 & .039 & .027 \\
\hline
\end{tabular}

Research Question 2 
To meet the needs of the current research questions, only clients who either prematurely terminated $(59 \%, n=35)$ or persisted $(41 \%, n=24)$ in counseling were included in the analysis. Analysis of the data revealed that only a small portion $(n=13)$ of clients attended between two and seven sessions and therefore were not included as a distinct group in the analyses. Of the adolescent files examined $(N=74)$, a total of 59 were included in the analysis. The sample consisted of 55.9\% ( $n=33)$ female and $44.1 \%(n=26)$ male. The mean age of the sample was $14.2(S D=1.65)$ and the mean academic grade in school was $8.54(S D=2.13)$. The participants identified the following ethnicities: White (71.2\%, $n=47)$, Hispanic/Latino(a) $(15.2 \%, n=10)$, Bi-racial (7.6\%, $n=5)$, African American (4.5\%, $n=3)$, and Asian $(1.5 \%, n=1)$. An examination of family household income revealed: $\$ 40,001+(37.3 \%, n=19)$, less than $\$ 15,000$ (21.6\%, $n=11)$, $\$ 20,001-\$ 30,000(15.7 \%, n=8), \$ 15,001-\$ 20,000(13.7 \%, n=7), \$ 30,001-$ $\$ 40,000(11.8 \%, n=6)$. Fifteen (22.7\%) of the participants did not report their household income. See Table D.8 for a summary of descriptive statistics.

Table D.8

Demographic Descriptive Statistics

\begin{tabular}{lcc}
\hline & \multicolumn{2}{c}{$(\boldsymbol{n}=\mathbf{5 9})$} \\
\cline { 2 - 3 } Demographic Variable & $\boldsymbol{M}$ & $\boldsymbol{S D}$ \\
\hline Age & 14.39 & 1.60 \\
Grade & 8.57 & 2.32 \\
\hline & & \\
\hline Gender & $\boldsymbol{N}$ & $\mathbf{\%}$ \\
$\quad$ Female & 38 & 57.6 \\
$\quad$ Male & 26 & 39.4 \\
Race/ethnicity & & \\
$\quad$ African American & 3 & 4.5 \\
$\quad$ Asian & 1 & 1.5 \\
Bi-racial & 5 & 7.6 \\
Hispanic/Latino(a) & 10 & 15.2 \\
$\quad$ White & 47 & 71.2 \\
& & \\
\hline
\end{tabular}




\begin{tabular}{lcc}
\cline { 1 - 2 } SES & & \\
$<\$ 15,000$ & 11 & 21.6 \\
$\$ 15,001-\$ 20,000$ & 7 & 13.7 \\
$\$ 20,001-\$ 30,000$ & 8 & 15.7 \\
$\$ 30,001-\$ 40,000$ & 6 & 11.8 \\
$>\$ 40,001$ & 19 & 37.3 \\
\hline
\end{tabular}

Research Question 2.a

In order to understand the differences among adolescents who prematurely terminated from counseling compared adolescents who persisted in counseling on each of the demographic and clinical variables, I conducted a series of univariate and multivariate comparison analyses. Table D.9 provides the profile of demographic variables and Table D.10 provides the descriptive statistics for each predictor variable.

Firstly, I examined the differences among demographic variables between the two groups, premature terminators and persisters. Table D.9 presents the descriptive statistics for the cases in each group. Due to the observed differences, I conducted a series of analyses to compare the demographic variables between adolescents who prematurely terminated from counseling and those who persisted in counseling. The first demographic variable examined was age. The mean age for adolescents who prematurely terminated from counseling $(M=14.69, S D$ $=1.49)$ was greater than the mean age of adolescents who persisted in counseling ( $M=13.50$, $S D=1.64)$. An independent sample $t$-test revealed a statistically significant difference in age, $t(57)=2.880, \mathrm{p}=.006$, with a moderate-large effect size $\left(\eta^{2}=.13\right)$. Further, the mean grade of premature terminators $(M=8.85, S D=2.31)$ appeared higher than the mean grade of persisters $(M=8.09, S D=1.81)$. A subsequent independent sample $t$-test revealed there was not a statistically significant difference in grade, $t(54)=1.324, \mathrm{p}=.191$.

A chi-squared test of independence was conducted to determine if there was a difference 
in gender between adolescents who prematurely terminated from counseling and those who persisted in counseling. The results from the analysis were statistically significant, $X^{2}(1)=5.97$, $p=.015$, with a moderate effect size $(\varphi=.32)$. Male adolescent clients were statistically significantly more likely to prematurely terminate from counseling and less likely persist in counseling then female adolescent clients.

A chi-squared test of independence was conducted to determine if there was a difference in ethnicity between adolescents who prematurely terminated from counseling and those who persisted in counseling. For purposes of this analysis, the ethnicity variable was categorized into two groups, dominant (White) and non-dominant (African American, Asian, Bi-Racial, Hispanic/Latino(a)). The results from the analysis were not statistically significant, $X^{2}(1)=3.26$, $p=.07$, indicating individuals from dominant and non-dominant populations were equally likely to persist or terminate prematurely.

Finally, a chi-squared test of independence was conducted to determine if there was a difference in reported family income between adolescents who prematurely terminated from counseling and those who persisted in counseling. For purposes of this analysis, the income variable was categorized into two groups, less than $\$ 40,000$ and more than $\$ 40,000$. Individuals who reported a family income of less than $\$ 40,000$ qualified for the sliding scale fee the counseling clinic. The results from the analysis were not statistically significant, $X^{2}(1)=.221, p$ $=.03$ at $\mathrm{p}<.638$. Family income was not different between adolescents who prematurely terminated from counseling and adolescents who persisted in counseling. 
Table D.9

Demographic Descriptive Comparisons

\begin{tabular}{|c|c|c|c|c|}
\hline \multirow[b]{2}{*}{ Demographic Variable } & \multicolumn{2}{|c|}{$\begin{array}{l}\text { Premature Terminators } \\
\qquad(n=35)\end{array}$} & \multicolumn{2}{|c|}{$\begin{array}{c}\text { Persisters } \\
(n=24)\end{array}$} \\
\hline & $M$ & SD & $M$ & $S D$ \\
\hline Age & 14.69 & 1.49 & 13.50 & 1.64 \\
\hline \multirow[t]{2}{*}{ Grade } & 8.85 & 2.31 & 8.09 & 1.81 \\
\hline & $N$ & $\%$ & $N$ & $\%$ \\
\hline \multicolumn{5}{|l|}{ Gender } \\
\hline Female & 15 & 42.9 & 18 & 75 \\
\hline Male & 20 & 57.1 & 6 & 25 \\
\hline \multicolumn{5}{|l|}{ Race/ethnicity } \\
\hline Asian & 0 & 0 & 1 & 4.2 \\
\hline African American & 2 & 5.9 & 0 & 0 \\
\hline Bi-racial & 1 & 2.9 & 5 & 20.8 \\
\hline Hispanic Latino(a)/ & 4 & 11.4 & 4 & 16.7 \\
\hline White & 28 & 80.0 & 15 & 58.3 \\
\hline \multicolumn{5}{|l|}{ SES } \\
\hline$<\$ 15,000$ & 8 & 22.9 & 2 & 8.3 \\
\hline$\$ 15,001-\$ 20,000$ & 4 & 11.4 & 2 & 8.3 \\
\hline$\$ 20,001-\$ 30,000$ & 6 & 17.1 & 3 & 12.5 \\
\hline$\$ 30,001-\$ 40,000$ & 3 & 8.6 & 3 & 12.5 \\
\hline$>\$ 40,001$ & 11 & 31.4 & 7 & 29.2 \\
\hline
\end{tabular}

Following the demographic analyses, I ran comparisons for each of the clinical predictor variables. The first predictor variable examined was Adolescent involvement in the intake. A chisquared test of independence was conducted to determine if there was a difference across the two groups. The results from the analysis were not significant, $X^{2}(1)=.002, p=.960$. For each group, approximately $70 \%$ of adolescents were not involved in their intake session, indicating there were no differences among the groups on adolescent involvement in the intake. 
Table D.10

Predictor Variable Descriptive Statistics

\begin{tabular}{|c|c|c|c|c|}
\hline Predictors & $\begin{array}{l}\text { Prematurely } \\
\text { Terminates } \\
(n=35)\end{array}$ & & $\begin{array}{l}\text { Persists } \\
(n=24)\end{array}$ & \\
\hline \multirow{2}{*}{$\begin{array}{l}\text { Adolescent involvement in } \\
\text { intake }\end{array}$} & No $=71.4 \%$ & Yes $=28.6 \%$ & No $=70.8 \%$ & Yes $=29.2 \%$ \\
\hline & Mean & $S D$ & Mean & $S D$ \\
\hline Time on wait list & 71.86 & 61.25 & 57.00 & 52.16 \\
\hline Internalizing Behaviors & 60.89 & 9.94 & $65.67 *$ & 11.12 \\
\hline Externalizing Behaviors & 60.17 & 10.04 & $66.64 *$ & 12.17 \\
\hline $\begin{array}{l}\text { Adolescent-Focused } \\
\text { Stress }\end{array}$ & $113.74^{* *}$ & 28.34 & 109.25 & 28.71 \\
\hline Parent-Focused Stress & 82.34 & 17.91 & 80.54 & 22.81 \\
\hline $\begin{array}{l}\text { Adolescent-Parent } \\
\text { Relationship }\end{array}$ & 38.74 & 11.77 & 38.71 & 10.23 \\
\hline Life Stressors & 3.29 & 2.21 & 3.92 & 2.48 \\
\hline
\end{tabular}

Differences between premature terminators and persisters were examined for the amount of Time spent on waitlist by conducting an independent sample t-test. Although the means of the two groups appeared different (premature terminators, $M=71.86$, $S D=61.25$; persisters, $M=$ $57.00, S D=52.16)$, the $t$-test revealed that differences in number of days spent on the waitlist were not statistically significantly different, $t(57)=1.00, \mathrm{p}=.32$.

A multivariate analysis of variance (MANOVA) was conducted to examine differences in Internalizing and Externalizing Behaviors for each of the groups, premature terminators and persisters. The data met the assumption for homogeneity of variance-covariance, Box's $M=$ $.487, p=.926$. Additionally, both variables met the assumption of equality of variances with values of $p=.971$ and $p=.489$ respectively. An examination of Wilks' Lambda $=.946, \mathrm{p}=.211$ revealed that there was not a statistically significant difference between internalizing and 
externalizing behaviors for adolescents who prematurely terminated from counseling and those who persisted in counseling. The mean scores for adolescents who persisted in counseling fell within the borderline clinical range for both internalizing $(M=65.67, S D=11.12)$ and externalizing $(M=66.64, S D=12.17)$ behaviors. See Table D.11 for the estimated marginal means.

Table D.11

Estimated Marginal Means

95\% Confidence Interval

Dependent

Variable

Internalizing

Problems

Externalizing

Problems
Groups

Premature

Termination

Persist

Premature

Termination

Persist
Std.

Mean Error Lower Bound Upper Bound

60.886

1.769

57.344

64.427

65.667

2.136

60.171

1.680

61.390

56.807

69.944

63.535

$61.167 \quad 2.029$
57.104
65.229

A subsequent MANOVA was conducted to examine differences across four levels of parental stress across the two groups. The predictor variables examined were: Adolescentrelated stress, Parent-related stress, Adolescent-Parent Relationship, and Life Stressors. The data met the assumption for homogeneity of variance-covariance, Box's $M=29.12, p=.003$ at the $p>.001$ level. Additionally, all variables met the assumption of equality of variances with values of $p=.817, p=.323, p=.417$ and $p=.680$ respectively. An examination of Wilks' Lambda $=.969, \mathrm{p}=.780$ revealed that there was no statistically significant difference in parentreported stress for adolescents who prematurely terminated from counseling and those who persisted in counseling. See Table D.12 for the estimated marginal means for each of the four groups and Table D.10 for the means and standard deviations for each of the groups. 
Table D.12

Estimated Marginal Means

95\% Confidence Interval

Dependent

\begin{tabular}{|c|c|c|c|c|c|}
\hline Variable & Groups & Mean & Std. Error & Lower Bound & Upper Bound \\
\hline $\begin{array}{l}\text { Adolescent-Focused } \\
\text { Stress }\end{array}$ & $\begin{array}{l}\text { Premature } \\
\text { Termination }\end{array}$ & 113.743 & 4.815 & 104.100 & 123.386 \\
\hline & Persist & 109.250 & 5.815 & 97.605 & 120.895 \\
\hline $\begin{array}{l}\text { Parent-Focused } \\
\text { Stress }\end{array}$ & $\begin{array}{l}\text { Premature } \\
\text { Termination }\end{array}$ & 82.343 & 3.387 & 75.561 & 89.124 \\
\hline & Persist & 80.542 & 4.090 & 72.352 & 88.731 \\
\hline $\begin{array}{l}\text { Adolescent-Parent } \\
\text { Relationship }\end{array}$ & $\begin{array}{l}\text { Premature } \\
\text { Termination }\end{array}$ & 38.743 & 1.889 & 34.959 & 42.526 \\
\hline \multirow{3}{*}{ Life Stressors } & Persist & 38.708 & 2.282 & 34.139 & 43.277 \\
\hline & $\begin{array}{l}\text { Premature } \\
\text { Termination }\end{array}$ & 3.286 & .382 & 2.521 & 4.050 \\
\hline & Persist & 3.917 & .461 & 2.993 & 4.840 \\
\hline
\end{tabular}

Research Question 2.b

A Predictive Discriminant Analysis (PDA) was conducted to examine the primary research question:

To what degree can gender, age, whether an adolescent is involved in their intake, time spent on the wait list, levels of parental stress, and parent perceptions of adolescent internalizing and externalizing behaviors predict whether an adolescent will terminate prematurely or persist in counseling?

The purpose of conducting a PDA is to determine to what extent a combination of variables can accurately predict group membership (Poulsen \& French, 2002). Ten predictor variables were examined to create a predictive model of adolescent persistence in counseling. Two distinct groups were examined, adolescents who prematurely terminated $(n=35)$ from counseling and adolescents who persisted $(n=24)$ in counseling. The overall Wilks' lambda was statistically 
significant $(\Lambda=.70, \mathrm{p}=.03)$, indicating that the predictors were able to discriminate between the groups at a rate greater than chance. Because of this, I decided to examine the model results.

In PDA, results are determined by an examination of the classification results matrix and the classification function coefficients (Huberty \& Olejnik, 2006). Examination of the classification results matrix provides an understanding of the overall predictability, or "hit rate," of the model (Huberty \& Olejnik, 2006). The hit rate informs the researcher how accurate the linear classification function was in accurately classifying the initial cases that were included in the model (Huberty \& Olejnk, 2006). A model with a high hit rate provides a framework to predict whether an adolescent client will either persist in counseling or prematurely terminate from counseling. The hit rate for the current model was $74.6 \%$, meaning that 44 of the 59 cases were correctly classified based on the model. Of the clients who persisted in counseling, 75\% ( $n$ $=18$ ) were predicted correctly; $74.3 \%(n=26)$ of adolescents who prematurely terminated were predicted correctly. An examination of the overall hit rate as well as the hit rate for each of the two groups reveals hit rates that are greater than what would be expected by chance (prematurely terminated 59.3\%, persisted, 40.7\%). See Table D.13 for the classification results matrix.

Table D.13

Classification Results

\begin{tabular}{|c|c|c|c|c|c|}
\hline & & \multirow[t]{2}{*}{ Actual Group } & \multicolumn{2}{|c|}{$\begin{array}{c}\text { Predicted Group } \\
\text { Membership }\end{array}$} & \multirow[b]{2}{*}{ Total } \\
\hline & & & $\begin{array}{c}\text { Premature } \\
\text { Termination }\end{array}$ & Persist & \\
\hline \multirow[t]{4}{*}{ Original } & Count & Premature Termination & 26 & 9 & 35 \\
\hline & & Persist & 6 & 18 & 24 \\
\hline & $\%$ & Premature Termination & 74.3 & 25.7 & 100.0 \\
\hline & & Persist & 25.0 & 75.0 & 100.0 \\
\hline
\end{tabular}

$69.5 \%$ of original grouped cases correctly classified. 
The classification function coefficients serve as the linear classification function for the classification of future cases (Huberty \& Olejnik, 2006). Multiplying the score of each predictor by the computed weight, adding the resulting products, and then adding the constant value achieve a linear composite score for a case. This computation results in two composite scores, with future cases being assigned to whichever group has the largest score (Huberty \& Olejnik, 2006). See Table D.14 for the classification function coefficients.

Table D.14

Classification Function Coefficients

\begin{tabular}{lcc}
\hline & \multicolumn{2}{c}{ Groups } \\
\cline { 2 - 3 } & Premature & \\
& Termination & Persist \\
\hline Gender & 3.876 & 5.866 \\
Age & 6.079 & 5.580 \\
Adolescent involvement in intake & -1.184 & -.753 \\
Internalizing Behaviors & .455 & .527 \\
Externalizing Behaviors & .531 & .522 \\
Parent-Focused Stress & -.031 & -.049 \\
Adolescent-Focused Stress & -.049 & -.047 \\
Adolescent-Parent Relationship Stress & -.032 & -.033 \\
Life Stressors & -.466 & -.305 \\
(Constant) & -70.387 & -67.952 \\
\hline
\end{tabular}


APPENDIX E

\section{DISCUSSION}




\section{Discussion}

The variables examined in this study were derived from an examination of previous research on counseling persistence. All analyses conducted provided critical information for counselors who provide counseling services to adolescent clients and their families. Although the predictive model was not found to be statistically significant, it was able to provide clinicians with a model to examine the likelihood of adolescent treatment persistence with a nearly $75 \%$ accuracy rate. Adolescents face an array of barriers in seeking out counseling, and the National Alliance on Mental Illness (2013) found that only 20\% of youth struggling with mental illness received appropriate treatment for their mental health concerns. When adolescents and their families seek out counseling, it is imperative for counselors to be able to provide support to ensure adolescents are able to persist. This chapter includes discussion regarding results that emerged from examination of demographic and clinical variables present within the model and provides suggestions on research and clinical implications for counselors.

Similar to previous findings, a review of demographic variables revealed variable differences between adolescents who prematurely terminated from counseling and adolescents who persisted in counseling. Age and gender were the only two variables with statistically significant differences between the groups; grade, ethnicity, and household income did not vary between the groups. Adolescents who prematurely terminated from counseling were notably older (14.69) than adolescents who persisted in counseling (13.50). This finding is inconsistent with previous researchers who found no relationship between client's age and treatment

persistence. These studies specifically examined persistence in children (Tsai \& Ray, 2011) and youth under 18 (Venable \& Thompson, 1998). As adolescent clients continue to increase their autonomy, the decision to continue to counseling may be less controlled by their parents, thus 
leading to higher rates of attrition among older adolescents. Because of this, it may be important for counselors to engage the adolescent clients in the counseling process from the intake session and encourage their autonomous decision to persist. Despite differences in age, there was not a significant difference in grade-level among the adolescent clients. This lack of consistency in the assumed relationship between age and grade may be attributed to the increased rates of retention experienced by adolescents who are seeking counseling services.

Another difference among the groups that emerged when examining demographic variables is the lack of treatment persistence among adolescent males. Nearly half of the adolescents in the sample were male, but only a quarter of the adolescents who persisted in counseling were male. Additionally, male clients were more likely to prematurely terminate from counseling. Although previous researchers found no relationship between gender and persistence in children (Kazdin \& Mazurick, 1994; Tsai \& Ray, 2011), this relationship was significant for the adolescent clients within the current study. The distinct difference between male and female adolescent clients may be attributed to the presence of externalizing behaviors characteristic of adolescent males (Baruch et al., 2009; Pagnin et al., 2004; Sunseri, 2003), which may have a more profound impact on treatment persistence as males transition from childhood to adolescence.

Researchers have found conflicting results regarding the relationships between among client ethnicity (Kazdin et al.,1995; Kazdin \& Mazurick, 1994; Tsai \& Ray, 2011), SES (Kazdin, 1990; Kazdin \& Mazurick, 1994; Prinz \& Miller, 1994; Tsai \& Ray, 2011), and treatment persistence. In the current study, no statistically significant differences were found between clients who identified as dominant and non-dominant ethnic groups. Further, no differences were found based on family income. It has been suggested that ethnicity and SES has an interaction 
effect when examining treatment persistence (Kazdin, et al., 1995). Similar to the clinical setting in Tsai and Ray’s (2001) study, the counseling clinic where the services were provided based on a sliding-scale fee. The ability for clients to receive affordable treatment may serve to counterbalance the impact of SES that has been identified in other studies (Kazdin, 1990; Kazdin \& Mazurick, 1994; Prinz \& Miller, 1994). Additionally, families were unable to report variance in income above $\$ 40,000$, which may have impacted the ability to fully understand impact of SES on persistence in counseling.

In the current study, no statistically significant differences were found between adolescents who prematurely terminated from counseling and those who persisted in counseling on the clinical variables assessed: adolescent involvement in the intake, time spent on the waitlist, internalizing behaviors, externalizing behaviors, and four areas of parental stress. Despite this, several findings emerged from examining the findings from both the regression analysis and the PDA. Although parents of adolescents were advised upon calling the clinics to bring their adolescent child to the intake session, less than a third of adolescents were actually involved in the initiation of their counseling services. The regression analysis revealed that adolescent involvement in the intake accounted for nearly $30 \%$ of the variance explained in the amount of counseling sessions attended. Further, adolescent involvement in the intake was positively correlated with number of sessions completed.

Although only minor differences were observed between adolescents who persisted in counseling and those who prematurely terminated from counseling, the overall low rate of involvement is meaningful from a clinical standpoint. Two clinical issues arise from this: lack of clinical information provided by the adolescent and lack of perceived involvement in the counseling process by the adolescent. Adolescence is a time of increase autonomy and profound 
changes in the parent-adolescent relationship (Silverberg \& Steinberg, 1990). Counselors and counseling centers that provide services to adolescents may wish to review their policies on early involvement of adolescents in their counseling process. Early involvement would allow adolescents to complete intake assessments and provide counselors with valuable clinical information. Additionally, it would provide the adolescent with an opportunity to be engaged in the counseling process rather than feeling as though he or she is being sent involuntarily. Early engagement would also provide the opportunity for the development of the therapeutic relationship with the adolescent, decreasing the risk that they view the counselor as an extension of a parental figure.

In this sample, the average time spent on the wait-list for adolescents who prematurely terminated (71.86 days) appeared substantially longer than the time spent on the wait-list for adolescents who persisted in counseling (57.00 days). The services provided at the clinics were based on a sliding-scale fee, which may have contributed to the extended wait times across both groups. Unlike Long at al.'s (1992) findings on adult persistence in counseling, the length of time on the waitlist did not appear to be a significant factor is explaining the number of sessions attended. It is unknown how this factor impacted families' decisions to engage in counseling; if the clients sought services elsewhere, if the distress they experienced became more manageable, or if they became discouraged with services and chose to not engage in counseling. Because clients often seek out counseling in times of crisis, it is imperative for counselors and clinics to be transparent with clients and their families about the potential length of time they will need to wait prior to receiving counseling services. In order to ensure that counselors are acting with beneficence, counselors must also provide adequate resources and referrals to clients if they are unable to provide timely services. 
Scores on the CBCL are categorized based on a continuum that ranges from: non-clinical (<65), to borderline clinical (65-70), and clinical (>70; Achenbach \& Rescorla, 2001). Both the internalizing (65.67) and externalizing (66.64) scores for adolescents who persisted were within the borderline clinical range. In the regression analysis, externalizing behaviors accounted for over $40 \%$ of the variance explained in number of sessions attended by the adolescent clients. Additionally, there was a negative relationship among the variables, suggesting that adolescents presenting with high levels of externalizing behaviors may be at risk of not persisting in counseling. While not as striking the relationship with externalizing behaviors, internalizing behaviors accounted for $12.96 \%$ of the variance in the predicted number of sessions attended. Previous researchers found that internalizing behaviors such as depression and anxiety were indicative of treatment persistence but externalizing behaviors such as delinquency and substance abuse were indicative of premature termination (Baruch et al., 2009; Pagnin et al., 2004; Sunseri, 2003). Thus, it is evident that an understanding of adolescents' presenting concerns, whether externalizing or internalizing in nature, would serve as a valuable assessment tool in assessing client's ability to persist in counseling.

In previous studies examining persistence in child and adolescent counseling, researchers found high levels of parental stress to be associated with premature termination (Attride-Stirling, Davis, Farrell, Groark, \& Day, 2004; Kazdin \& Mazurick, 1994; Kazdin, Stolar \& Maciano, 1995; Prinz \& Miller, 1994; Tsai \& Ray, 2011). Although the levels of parental stress in the current study were generally higher for adolescents who prematurely terminated, unlike previous findings, the levels of stress were not significantly higher for these clients. Further, the only parental stress score that was in the borderline clinical range was the adolescent-focused stress of parents of adolescents who prematurely terminated from counseling (Sheras et al., 1998). 
Although parents presented with high levels of stress, they rarely reported clinical levels of stress at the time of intake. Results from the regression analysis revealed that life stressors and adolescent-parent relationship were positively associated with the number of sessions attended, while parent-focused stress and adolescent-focused stress were negatively associated with the number of sessions attended. Further, parent-focused stress accounted for $10.30 \%$ of the variance explained in the number of sessions attended. Together, this suggests that adolescents with parents who are experiencing high levels of stress related to their parental role attend fewer counseling sessions.

A review of the literature and current findings does not provide clarity in the meaning behind this difference beyond the observation that external life events such as a divorce or a death in the family may trigger parents to seek out counseling services for their adolescents. It is possible distress experienced due to an identified external event may take precedence over expressed barriers to treatment such as embarrassment or shame (Yap et al., 2012). Overall, the findings from the current study were consistent with previous research; parent stress can be a limiting factor in adolescent persistence in counseling. Thus, it is imperative for counselors to provide appropriate support and resources to parents to increase the likelihood that adolescents will continue in counseling. This support may include identifying counselors to provide counseling to parents, holding parent support groups, or providing resources for parents to seek out external support. Researchers also found parental engagement to be an important factor in their commitment to the counseling process (Kazdin et al., 1997). The inclusion of parentconsultations and the incorporation of psychoeducation may provide parents with the support needed to adhere to counseling. 
The purpose of a PDA is to create a predictive model that can be utilized by counselors to identify adolescents who are at risk of prematurely terminating from counseling. Specifically, the current model serves to predict whether an adolescent client will attend either just the intake session and/or the first counseling session or persist through at least eight counseling sessions, which has been identified as being the point clients begin to experience therapeutic change (Lambert, 2013). The classification function coefficients provided in Table 14 provides counselors or agencies with an equation that can be applied to future clients to determine the likelihood that an adolescent will either prematurely terminate or persist in counseling. If scores of all variables are available, counselors may complete the following steps to determine which group the adolescent client most closely fits: (1) Multiply the score of each predictor by the computed weight, (2) Add the resulting products, (3) Add the constant value achieve a linear composite score for a case. This computation results in two composite scores, with future cases being assigned to whichever group has the largest score (Huberty \& Olejnik, 2006). While this model is useful as an additional screening tool, it is not without error and should not replace counselor's clinical judgment in identifying clients at-risk of prematurely terminating. Continued examination of the model may provide additional insight into the most predictive variables, thus simplifying the assessment procedure.

Overall, the findings from the current study are consistent with previous research on the relationship between the nature of clients' presenting problems on persistence in counseling but was not supportive of previous findings on the impact of parental stress. The early identification of adolescents who present as male, report high levels of externalizing behaviors, or are from homes characterized by high levels of parental stress provide counselors with key identifiers to engage in early-intervention strategies to increase persistence in counseling. Further, the 
predictive model created may serve as a screening tool to identify adolescents who are at risk of premature termination from counseling.

Implications for Research

While the current study sought to examine variables associated with adolescent persistence in counseling, several implications for future research emerged. These include follow-up quantitative and qualitative analyses examining variables related to the client, family, counselor, and counseling experience. Further, research implications for examining counseling persistence across the lifespan are discussed.

Although the variables selected for the current study were derived from an analysis of current literature, the utilization of archival data served as a limitation in available client variables. Because of this, researchers may examine additional client variables. For example, youth self-reported presenting concerns, diagnosis, adolescent's involvement in social or extracurricular activities, and variables related to their academic performance may provide additional insight into the profile of adolescents who may prematurely terminate from counseling. Additionally, researchers may also examine variables related to the family system such as single-parent status, mental health concerns, and involvement in the counseling process.

There are also opportunities for qualitative exploration of the experience of both the adolescent clients and their families. It may be helpful for researchers to interview adolescents and their families on their experience if they either persisted in counseling or prematurely terminated from counseling. Obtaining this information directly from the individuals would allow counselors and researchers to better understand the experience of individuals that impacted their decision regarding counseling. Allowing this open forum would provide clients and their 
families opportunities to discuss experiences that encouraged their attendance or served as a barrier in their continued attendance.

Analysis of the counselor and counseling experience may also be important. Variables related to the counselor, including their race, ethnicity, sexual orientation, and levels of experience may impact client persistence in counseling. Variables related to the counseling process, such as working alliance, theoretical orientation, techniques, and duration and frequency of sessions could also be explored. Additionally, researchers could analyze clinical information from weekly progress notes to understand the impact that the development of the therapeutic relationship has on client persistence.

Further, counselors would benefit from additional research on counseling persistence across the lifespan and across treatment modalities. It would be valuable for researchers to examine variables specific to developmental ranges to help inform counselor's of clients who may be at risk for prematurely terminating from counseling. An examination of factors specific to treatment settings (i.e., outpatient, group counseling, inpatient, residential) would also serve to assist counselors in implement early-intervention strategies to promote persistence in counseling. Implications for Clinical Practice

For counselors working with adolescents, several direct clinical implications emerge from the current findings. These implications range from the initial assessment procedures and continue through the counseling process. Additional implications for family support are also discussed.

The process of an adolescent receiving counseling services typically begins with their parents scheduling an initial intake session. For counselors, this is the first opportunity to encourage early-involvement of the adolescent in the counseling process. Although still legally 
minors, adolescents are beginning to have increased autonomy and responsibility, and their inclusion in the intake process provides counselors with the opportunity to promote this developmental process. It is essential for counselors to be intentional in collecting clinical information and assessments from not only parents, but also adolescents. This serves two purposes: to increase the likelihood of getting an accurate clinical understanding of the presenting concerns and to communicate the adolescent's personal investment in the counseling process. The early establishment of mutual trust and respect may serve to foster the development of the therapeutic relationship and encourage persistence in counseling.

Counselors may also find it helpful to incorporate the predictive model developed in the current study into their assessment procedures. Dependent on the assessments utilized, if all clinical variables are available (gender, age, adolescent involvement in the intake, days spent on the waitlist, internalizing and externalizing behaviors, and the four domains of parental stress), counselors may input the scores into the classification function (see Table 14) to inform their understanding of clients who may be at risk for prematurely terminating from counseling.

Further, the identification of clients who are at risk for prematurely terminating from counseling may provide counselors with the ability to engage in early-intervention strategies. These strategies may include providing conjunctive counseling or psychoeducation to parents, in addition to providing information on available community resources. Counselors may also choose to share the information derived from the model with the adolescent and their parents to discuss the identified risk factors and work collaboratively to address any barriers to treatment.

\section{Limitations}

There are several limitations to the current study. Firstly, due to utilization of archival data, the instruments analyzed were pre-determined and administered to the clients. Because of 
this, parents or guardians completed all information and assessments based on their knowledge of the adolescent's behaviors or experiences. Consequently, no information was available based on the adolescents' personal perception of their concerns. Rescorla et al. (2007) found that adolescent self-report measures revealed higher levels of distress than measure completed by parents. Due to increased autonomy during adolescence, the lack of adolescent self-report information leads to valuable information regarding adolescents' true experiences being missed. The utilization of self-report measures also presents the risk of social desirability, which may lead to results that account for error rather than true group differences. Future studies may examine assessments completed by adolescent clients, as well as examine other means of information, including the content of counselor progress notes or counselor characteristics.

The utilization of archival data also resulted limited the available sample size. Although substantially more adolescent files were identified, a large number of the files were ineligible for inclusion due to counselors either not following appropriate assessment protocols or incomplete data. A larger sample size may serve to increase the power of the predictive model and provide additional insight into the differences between adolescents who prematurely terminate from counseling and those who persist in counseling.

An additional limitation is a result of the lack of standardization across research in defining premature termination and persistence. Previous researchers defined terms based on personal clinical judgment. As a consequence of this lack of clarity, the current study did not examine the characteristics of adolescents who did not meet the criteria for premature termination or persistence. These clients, who attended between two and seven sessions, fell into a middle group that was not considered to have persisted to the point of significant benefit from treatment (Lambert, 2013). Further analysis of these adolescents was also limited by the small 
sample size available $(n=13)$. Additional research on trends of attrition and clinical improvement is needed to provide future researchers with guidance on standardization of terms. Due to the presence of developmental differences, research specifically examining treatment length for children and adolescents would also provide counselors and researchers with valuable insight.

Generalizability of findings is limited due to all of the clients being from the same geographic location. It should also be noted that counseling services were provided at a training clinic with no standardized treatment approach, which may have impacted the results of the study. Experience of the counselors ranged from master's level interns to doctoral level practitioners. Researchers have explored the impact of counselor characteristics on client's experience of counseling and found conflicting results in regard to counsel's level of experience and treatment persistence (Lampropoulos et al., 2009; Long et al., 1998). Despite this, an examination of adolescent client files from a range of treatment facilities, including agencies and private practices, would provide additional information regarding the predictability power of counselor and counseling variables when examining adolescent treatment persistence.

Although the current model was developed based on previous literature on attrition, it is possible that other variables, such as counselor characteristics or perceived available social support, may also be valuable in understanding treatment persistence. Future researchers may examine the impact of these other variables in determining the most efficient model to predict persistence in counseling.

\section{Conclusion}

Adolescents often experience distress during this critical developmental period and may benefit from receiving counseling services. Beyond the barriers experienced in seeking out 
counseling, counselors struggle with high rates of premature termination among their clients. Results from the current study indicate that the presence of externalizing behaviors may be the strongest indicator of adolescents who are at risk of prematurely terminating counseling. Involving adolescents in the intake process may also serve to increase persistence in counseling as well as provide additional clinical information to counselors to accurately assess the adolescent's presenting concerns. The utilization of the predictive model may serve as a tool for counselors to aid in the early identification of adolescents who are at risk for premature termination. 
APPENDIX F

SUPPLEMENTAL MATERIALS 


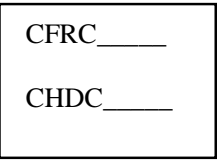

University of North Texas

Department of Counseling and Higher Education Counseling Program Clinical Services

\section{Child/Adolescent Background Information (use for all minors)}

Welcome to the Child and Family Resource Clinic. Please answer all information as completely as possible. If applicable, both mother and father should complete together. Information given is strictly confidential and beneficial in providing the best possible service. Feel free to ask for assistance, if needed. Your child's counselor will discuss your responses with you after he/she has reviewed the form.

Child's Name:

Date of First Visit:

Completed by:

Cell Phone:

Home Phone:

Work Phone:

Best Time and Place to call:

Child's Address:

Last

Street

(12)Child's Gender: Male Female

(13) Age Relationship to Child:

(May call? Yes No May Leave Message? Yes No) (May call? Yes No May Leave Message? Yes No) (May call? Yes No May Leave Message? Yes No)

(15)Child's Ethnicity:

African American_

Bi-racial

Hispanic/Latin

Asian

White

Native American

Other

Child's primary language: English ___ Spanish ___ Other

Language spoken at home (parent's language):

Child's Legal Guardian (Managing Conservator):

(If the child is not living with both natural parents, both adoptive parents, or only living parent, the clinic requires a photocopy of the legal document stating custody arrangements, consisting of the cover page, page specifying conservator(s), and signature page). (The photocopy should be stapled to this form.)

In case of emergency, contact:

Name: Last, First $\quad$ Relationship Phone

Is your child presently receiving counseling elsewhere? Yes No

(If yes, do not complete this form until you have talked with your counselor)

Family members receiving services at this clinic? Yes No (Name/Dates of service)

Is your child currently on probation? Yes No School Child attends:

Current School Address \& Phone:

Grade Level (now):

Current Teacher(s): 1)

Current School Counselor:
Has your child ever been retained? Yes No 2) 3)

(16) Is your child receiving special education or other services? Yes No (explain)

Has your child ever seen a mental health professional (psychiatrist, psychologist, or a counselor)? Yes No (If so, we will need your permission in order to communicate with that individual or agency)

Previous Mental Health Professional/Agency: 
Phone:

Dates of Service:

(beginning - ending)

Has your child been hospitalized for mental health concerns? Yes No

If yes: When Where

How were you referred to our clinic? (Check those that apply):

Counselor/Psychologist/Psychiatrist

Court

DPRS

Flyer

Friend or Co-Worker

Minister

Newspaper Ad_

Physician

Relative
School personnel_

Self

UNT Community

Yellow Pages

Other

Are you seeking services because your child is a victim of a crime? Yes No

Did it result in legal action? Yes No (If Yes, explain)

Person responsible for financial arrangements with our clinic:

Are you applying for sliding scale payments? Yes No

(17)Gross Household Annual Income (including Child Support Payments)

Less than $\$ 15,000 \_15,000-20,000 \_\quad 20,001-30,000$

$40,001+$

(18)How many family members currently reside in your home?

\section{* INFORMATION ON CHILD’S MOTHER *}

Mother's Name:

Last

First

MI

(19) I am: _ biological mother stepmother adoptive mother other

Address:

Street

City

State

Zip

Home Phone: (May call: Yes No Leave Message: Yes No )

Work Phone: (May call: Yes No Leave Message: Yes No)

Date of Birth:

Employer:

Occupation: How Long:

(20)Education Level of Mother:

8th grade or below

High School

Trade School/Some College GED
Undergraduate Degree Graduate Degree

History of learning, emotional, or behavioral problems: Yes No

(If yes, please explain)

History of alcohol/drug/substance abuse: Yes No

(If yes, please explain)

History of family violence: Yes No

(If yes please explain) 
History of criminal activity: Yes No

(If yes, please explain)

(21) Current living arrangements:

Family of origin Single

Spouse/Partner ___ Roommate___ Other

(22) Marital Status (indicate all that apply and duration of each, ex. 1965-1985):

Never married

Currently married

Divorced

Widowed

Deceased

(23) Marital History

Number of Marriages

Number of Divorces

\section{* INFORMATION ON CHILD'S FATHER *}

Father's Name:

(24) I am

Last

Address:

Street

biological father

stepfather

First

adoptive father other

City

State

Zip

Home Phone:

Yes No)

(May call: Yes No Leave Message: Yes No)
Work Phone:

(May call: Yes No Leave Message:
Date of Birth:

Employer:

(25)Education Level of Father

8th grade or below

High School

History of learning, emotional, or behavioral problems: Yes No

(If yes, please explain)

History of alcohol/drug/substance abuse: Yes No

(If yes, please explain)

History of family violence: Yes No

(If yes please explain)

History of criminal activity: Yes No

(If yes, please explain)

(26) Current living arrangements:

Family of origin Single

Spouse/Partner

Roommate

Other

(27) Marital Status (indicate all that apply and duration of each, ex. 1965-1985):

Never married

Currently married

Divorced

Widowed

Undergraduate Degree

Graduate Degree

(28) Marital History

Number of Marriages

Number of Divorces 
(29) Child's current household:

Adoptive parents

Father only

Foster family

Institution

Mother only

\section{* GENERAL INFORMATION *}

Biological Father and Stepmother

Biological Mother and Stepfather

Biological Parents

Relatives (specify)

Grandparents

List by Household your child's current family, beginning with the oldest member and include the child:

Primary Household (anyone who currently lives with child)

How long in this current living situation:

Name

Age Gender

Relationship to you (include step, half, etc.)

Child lives in: House___ Apartment ___ Duplex ___ Other

Second Household (non-custodial or extended family - if applicable)

Name

Age Gender Relationship to you (include step, half, etc.)

Currently involved in a custody dispute: No Yes (If yes, explain)

If divorced, circle the number which best describes your relationship with your ex-spouse.

$\begin{array}{lccc}\text { Hostile } & \text { Frustrating } & \text { Friendly } \\ 1 \_ & 3 & 3 & 5\end{array}$

How often does client see non-custodial parent?

Child's Primary Care Physician:

* CHILD'S HEALTH *

Address

Name Phone


Has your child ever seen a psychiatrist? Yes No

Is child currently seeing a psychiatrist? Yes No (If yes, list name, address and phone):

\begin{tabular}{llll}
\hline \multicolumn{3}{c}{ Name } & Phone \\
\hline \multicolumn{1}{c}{ Address } \\
Date of LAST complete physical: \\
Physical Disability: Yes $\quad$ No & (If yes, explain) \\
Chronic Illness: & Yes & No & (If yes, explain) \\
Terminal Illness: $\quad$ Yes & No $\quad$ (If yes, explain)_
\end{tabular}

Check the following items for a diagnosis or medication that your child is now receiving or has received:

\begin{tabular}{|c|c|c|c|c|c|}
\hline Diagnosis & $\begin{array}{l}\text { Current } \\
\text { (list dates) }\end{array}$ & $\begin{array}{l}\text { Past } \\
\text { (list dates) }\end{array}$ & Physician's Name & Name of medication & Dosage \\
\hline \multicolumn{6}{|l|}{ Depression } \\
\hline \multicolumn{6}{|l|}{ ADHD } \\
\hline \multicolumn{6}{|l|}{ Conduct } \\
\hline \multicolumn{6}{|l|}{ Disorder } \\
\hline \multicolumn{6}{|l|}{ Anxiety/ } \\
\hline \multicolumn{6}{|l|}{ Nervousness } \\
\hline \multicolumn{6}{|c|}{ Manic-Depression } \\
\hline (Bipolar) & & & & 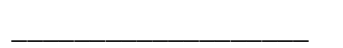 & \\
\hline \multicolumn{6}{|c|}{ Schizophrenia _ } \\
\hline \multicolumn{6}{|l|}{ Oppositional } \\
\hline \multicolumn{6}{|c|}{ Defiant Disorder } \\
\hline Mood/Anger & & & & & \\
\hline Tics & & & & & \\
\hline
\end{tabular}


Insomnia/

Sleeplessness

Obsessive/

Compulsive

Addictions

Seizures

Post-Traumatic

Stress Disorder

Other

(If you do not know the name and dosage of current medication, please bring the medication to your next session)

What other medication is your child currently taking?

Medication

Dosage

Taken for what reason?

\section{* CURRENT CONCERNS *}

(30) Circle the item that you see as the most significant issue for your child. Underline any additional concerns.

Problems Related to Abuse

Current or past physical abuse

Current or past sexual abuse

Current or past emotional abuse

Current or past neglect

History of abandonment

Suspected sexual abuse

History of family domestic violence

\section{$\underline{\text { Mood-related Concerns }}$}

Disturbing memories

Difficulty going to sleep/staying asleep

Nightmares/night terrors

Suicidal ideation

Sadness

Depression

Feelings of guilt and shame

\section{Academic/School Problems}

Learning difficulties

Problems with peers

Problems with teachers

Speech Problem

\section{Family Relationship Concerns}

Difficulty adjusting to family changes

Discipline concerns

Parent-child relationship problems

Sibling concerns

Divorce/Separation

Religious/Spiritual Concerns 
Excessive worrying

Anger/Irritable

$\underline{\text { Rule-Breaking/Behavior Problems }}$

Other Behavioral Concerns

Aggression toward others

Sexual identity concerns

Drug/alcohol use

Inappropriate sexual behavior

Truancy

Gang involvement

Overeating/refusal to eat

Bedwetting or soiling

Running away

Stealing

Hyperactive/Inattentive

Intentionally hurting animals

Fire-setting

Other Unusual Behaviors

(Please specify)

* Remember to circle the most significant issue.

When did you first become concerned about the main/most significant

issue?

How have you attempted before now to deal with this issue?

Other treatment your child has received to address any of the concerns indicated above: None

Couples Counseling

Group counseling

Individual counseling

Family counseling

Hospitalization

Other

\section{* HISTORY OF TRAUMA/STRESSORS RELATAED TO THE CHILD *}

(For each of the following items that apply, write in your child's approximate age at the time it occurred):

Chronic illness of family member

Death of significant person

Domestic Violence

Family member absent (explain)

Family member's disability/major accident/illness

Family member emotional problems (explain)

Family member suicide (explain)

Parents divorced

Child separated from parent (how long and when)

Death of a pet__ Difficult medical treatments

Victim of trauma (unusual, terrifying experience)

Sexual Assault

Other

History of your child having learning, emotional, behavioral problems: Yes No

(If yes, please explain)

History of your child having alcohol/drug/substance abuse: Yes No

(If yes, please explain)

History of family violence: Yes No

(If yes, please explain)

History of criminal activity in the family: Yes No

(If yes, please explain) 
Has your child been abused (check all that apply): Physically

Emotionally

Sexually

Has your child been neglected (check all that apply): Physically

Emotionally

School Problems (check all that apply):

Academic problems__ Discipline problems___ Social Problems___ Other

Early Language/Speech Problems (explain)

History of health/physical problems includes: (check all that apply):

Asthma

Disability

Nervous stomach

Bedwetting

Dizziness

Neurological problems/exam

Bone/joint/muscle

Severe Headaches

Severe PMS

Chest pain

Heart Palpitations

Serious overeating/under-eating

Chronic illness

Hospitalization

Shortness of breath without exertion

Developmental delay(s)

Major accident

Sleep problems

Chronic Diarrhea

Major illness

Surgeries

Other

* HOME ATMOSPHERE *

Your child's current use of Computer, VCR, and Television (circle the number of hours that best describes use):

Computer (circle approximate hours spent each week)

$$
\begin{array}{lllll}
0-2 & 3-5 & 6-8 & 9-11 & 12+
\end{array}
$$

TV/VCR (circle approximate hours spent each week)

\begin{tabular}{lllll}
$0-2$ & $3-5$ & $6-8$ & $9-11$ & $12+$ \\
\hline
\end{tabular}

What do you enjoy most about this child?

What do you find most difficult about this child?

Anything else you think we need to know?

What is the one thing I need to know to help your child today? 


\section{INTAKE SUMMARY REPORT}

Client's Name

Home Phone

Parent's Name
Age Date of Intake
Cell Interviewer

Fee Assessed

List ALL times client is available for counseling:

Monday

10:30-12:30
Tuesday

10:30-12:30
Wednesday

10:30-12:30
Thursday

10:30-12:30

Nature of concerns: Check all that apply. Circle appropriate qualifier to the right of symptom/problem where applicable.

\section{Circle Qualifier}

Suicide $++\quad$ Current: yes / no

Self injury ++ Current: yes / no

Aggression ++

Abuse ++

Current: yes / no

Current: yes / no

Drug/Alcoholism

Current: yes / no
Past: yes / no

Past: yes / no

Past: yes / no

Past: yes / no

Past: yes / no $\underline{\sqrt{ } \text { yes }}$

__ Anxiety

Depression

Eating Disorder

Death/Loss

Divorce $\underline{\sqrt{\text { yes }}}$

__ Family Concerns

__ Custody Concerns

__ Parenting Concerns **

__ Child Behavior Problems *

__ School/Education problems*

_ Couple/Partner Concerns _ _ Career Concerns

Other Concerns

Is client employed student disabled other

Brief mental health history or N/A 


\section{COMPREHENSIVE REFERENCE LIST}

Abidin, R. R. (1993). Parenting stress index clinical manual. Charlottesville, Virginia: Pediatric Psychology Press.

Achenbach, T. M., \& Rescorla, L. (2001). Manual for the ASEBA school-age forms and profiles. Burlington: University of Vermont, Research Center for Children, Youth, \& Families.

American Counseling Association (2010). 20/20: A vision for the future of counseling. Retrieved from: http://www.counseling.org/knowledge-center/20-20-a-vision-for-the-future-ofcounseling/consensus-definition-of-counseling

American Psychiatric Association. (2000). Diagnostic and statistical manual of mental disorders (4th ed., text rev.). Washington, DC: Author.

American Psychiatric Association. (1994). Diagnostic and statistical manual of mental disorders (4th ed.). Washington, DC: Author.

Arnett, J. J. (2012). Adolescence and emerging adulthood (5 ${ }^{\text {th }}$ ed.). Upper Saddle River, New Jersey: Pearson.

Arnett, J. J. (2004). Adolescence and emerging adulthood ( $2^{\text {nd }}$ ed.). Upper Saddle River, New Jersey: Pearson.

Attride-Stirling, J., Davis, H., Farrell, L., Groark, C., \& Day, C. (2004). Factors influencing parental engagement in a community child and adolescent mental health service: A qualitative comparison of completers and non-completers. Clinical Child Psychology and Psychiatry, 9, 347-361. doi:10.1177/1359104504043918

Ausubel, D. P. (2002). Theory and problems of adolescent development ( $3^{\text {rd }}$ ed.). Lincoln, NE: Writers Club Press. 
Baruch, G., Vrouva, I., \& Fearon, P. (2009). A follow-up study of characteristics of young people that dropout and continue psychotherapy: Service implications for a clinic in the community. Child and Adolescent Mental Health, 14(2), 69-75. doi:10.1111/j.1475 $-3588.2008 .00492 . x$

Bastien, R. T., \& Adelman, H. S. (1984). Noncompulsory versus legally mandated placement, perceived choice, and response to treatment among adolescents. Journal of Consulting and Clinical Psychology, 52(2), 171-179. doi:10.1037/0022-006X.52.2.171

Beharry, M. S. (2013). Adolescent sexuality. Pediatric Annals, 42, 64-65. doi:10.3928/ 00904481-20130128-07

Behr, M., Nuding, D., \& McGinnis, S. (2012). Person-centered psychotherapy and counseling with children and young people. In Cooper, M., O’Hara, M., Schmid, P. F., \& Bohart, A. (Eds.) (2013). The handbook of person-centered psychotherapy and counseling ( $2^{\text {nd }}$ ed., pp. 266-281). New York, NY: Palgrave Macmillan.

Belfer, M. L. (2008). Child and adolescent mental disorders: The magnitude of the problem across the globe. Journal of Child Psychology and Psychiatry 49(3), 226-236. doi: 10.1111/j.469-7610.2007.01855.x

Blotcky, A. D., \& Friedman, S. (1984). Premature termination from psychotherapy by adolescents. Journal of Clinical Child Psychology, 13(3), 304-309. doi: $10.1080 / 15374418409533207$

Boldero, J., \& Fallon, B. (1995). Adolescent help-seeking: What do they get help for and from whom? Journal of Adolescence, 18, 193-209. doi:10.1006/jado.1995.1013 
Callahan, J. L., Aubuchon-Endsley, N., Borja, S. E., \& Swift, J. K. (2009). Pretreatment expectancies and premature termination in a training clinic environment. Training and Education in Professional Psychology, 3(2), 111-119. doi:10.1037/a0012901

Chang, H., \& Saunders, D. G. (2002). Predictors of attrition in two types of group programs for men who batter. Journal of Family Violence, 17(3), 273-292. doi:10.1023

$$
\text { /A:1016057328929 }
$$

Cohen, J. (1992). A power primer. Psychological Bulletin, 112, 155-159. doi:10.1037/0033 $-2909.112 .1 .155$

Del Mauro, J. M., \&, D. J. (2012). Children and adolescent’s attitudes toward seeking help from professional mental health providers. International Journal of Advanced Counseling, 35, 120-138. doi: 10:1007/s10447-012-9172-6

Dierker, L., Nergiso, J., Wiseman, R., \& Hoff, D. (2001). Factors predicting attrition within a community initiated system of care. Journal of Child and Family Studies, 10(3), 367-383. doi:10.1023/A:1012581027044

Enea, V., \& Dafinoiu, I. (2009). Motivational/solution-focused intervention for reducing school truancy among adolescents. Journal of Cognitive \& Behavioral Psychotherapies, 9(2), 185-198.

Erikson, E. H. (1968). Identity: Youth and crisis. New York, NewYork: Norton.

Fallon, B. J., \& Bowles, T. V. P. (2001). Family functioning and adolescent help seeking behavior. Family Relations, 50(3), 239-245. doi:10.1111/j.1741-3729.2001.00239.x

Faul, F., Erdfelder, E., Buchner, A., \& Lang, A. G. (2009). Statistical power analyses using G*Power 3.1: Tests for correlation and regression analyses. Behavior Research Methods, 41, 1149-1160. doi:10.3758/BF03193146 
Field, A. (2009). Discovering statistics using SPSS ( ${ }^{\text {rd }}$ ed.). London, England: Sage.

Fortenberry, J. D. (2013). Puberty and adolescent sexuality. Hormones and Behavior, 64, 280287. doi:10.1016/j.yhbeh.2013.03.007

Fry, D. A., Messinger, A. M., Rickert, V. I., O’Connor, M. K., Palmetto, N., Lessel, H., \& Davidson, L. L. (2013). Adolescent relationship violence: Help-seeking and help-giving behaviors among peers. Journal of Urban Health, 91(2), 320-334. doi:10.1007/s11524 $-013-9826-7$

Guterman, N. B., Cameron, M., Hahm, H. C. (2003). Community violence exposure and associated behavior problems among children and adolescents in residential treatment. Journal of Aggression, Maltreatment \& Trauma, 6(2), 111-135. doi:10.1300 /J146v06n02_06

Hatchett, G. T. (2004). Reducing premature termination in university counseling centers. Journal of College Student Psychotherapy, 19(2), 13-27. doi:10.1300/J035v19n02_03

Hatchett, G. T. (2011). Training counseling students to work more effectively with short-term clients. International Journal of Advanced Counseling, 33, 206-215. doi: 10.1007 /s10447-011-9124-6

Hatchett, G. T., \& Park, H. L. (2003). Comparison of four operational definitions of premature termination. Psychotherapy: Theory, Research, Practice, Training, 40(3), 226-231. doi: 10.1037/0033-3204.40.3.226

Harris, P. M. (1998). Attrition revisited. American Journal of Evaluation, 19(3), 293-305. doi: $10.1177 / 109821409801900303$

Henson, R. K. (1999). Multivariate normality: What is it and how is it assessed? In B. Thompson (Ed.), Advances in social science methodology (Vol. 5, pp. 193-211). Stamford, CT: JAI. 
Huberty, C. J., \& Olejnik, S. (2006). Applied MANOVA and discriminant analysis ( $2^{\text {nd }}$ ed.). Hoboken, NJ: Wiley-Interscience.

Husky, M. M., McGuire, L., Flynn, L., Chrostowski, C., \& Olfson, M. (2009). Correlates of help-seeking behavior among at-risk adolescence. Child Psychiatry and Human Development, 40, 15-24. doi:10.1007/s10578-008-0107-8

Jensen, C. D., Cushing, C. C., Aylward, B. S., Craig, J. T., Sorell, D. M., \& Steele, R. G. (2011). Effectiveness of motivational interviewing interventions for adolescent substance use behavior change: A meta-analytic review. Journal of Consulting and Clinical Psychology, 79(4), 433-440. doi:10.1037/a0023992

Kadera, S. W., Lambert, M. J., \& Andrews, A. A. (1996). How much therapy is enough? A session by session analysis of the psychotherapy dose-effect relationship. Journal of Psychotherapy Practice and Research, 5(2), 132-151.

Kazdin, A. E. (1990). Premature termination from treatment among children referred for antisocial behavior. Journal of Child Psychology \& Psychiatry \& Allied Disciplines, 31(3), 415-425. doi:10.1111/j.1469-7610.1990.tb01578.x

Kazdin, A. E., Holland, L., \& Crowley, M. (1997). Family experience of barriers to treatment and termination from child therapy. Journal of Consulting and Clinical Psychology, 65(3), 453-463. doi:10.1037/0022-006X.65.3.453

Kazdin, A. E., \& Mazurick, J. L. (1994). Dropping out of children psychotherapy: Distinguishing early and late dropouts over the course of treatment. Journal of Consulting and Clinical Psychology, 62, 1069-1074. 
Kazdin, A. E., Stolar, M. J., \& Marciano, P. L. (1995). Risk factors for dropping out of treatment among white and black families. Journal of Family Psychology, 9(4), 402-417. doi: 10.1037/0893-3200.9.4.402

Kessler, R. C., Berglund, P., Demler, O., Jin, R., Merikangas, K R., \& Walters, E. E. (2005). Lifetime prevalence and age-of-onset distributions of DSM-IV disorders in the national comorbidity survey replication. Archives of General Psychiatry, 62, 593-602. doi: 10.1001/archpsyc.62.6.593

Klecka, W. R. (1980). Discriminant analysis. Beverly Hills, CA: Sage.

Kraha, A. Turner, H., Nimon, K., Zientek, L. R., \& Hensen, R. K. (2012). Tools to support interpretations multiple regression in the face of multicollinearity. Frontiers in Psychology, 3, Article 44, 1-16. doi:10.3389/fpsyg.2012.00044

Lambert, M. J. (2013). The efficacy and effectiveness of psychotherapy. In M. J. Lambert (Ed.), Bergin and Garfield's handbook of psychotherapy and behavior change ( $6^{\text {th }}$ ed., pp. 169218). New York, NY: Wiley.

Lampropoulos, G. K., Schneider, M. K., \& Spengler, P. M. (2009). Predictors of early termination in a university counseling training clinic. Journal of Counseling and Development, 87, 36-46. doi:10.1002/j.1556-6678.2009.tb00547.x

Lerner, R. M. (2002). Adolescence: Development diversity, context, and application. Saddle River, New Jersey: Pearson Education.

Levy, J. J., Thompson-Leonardelli, K., Smith, N. G., \& Coleman, M. N. (2005). Attrition after intake at a university counseling center: Relationship among client race, problem type, and time on a waiting list. Journal of College Counseling, 8, 107-117. doi: 10.1002/j.2161-1882.2005.tb00077.x 
Longo, D. A., Lent, R. W., \& Brown, S. D. (1992). Client variables in the prediction of client motivation and attrition. Journal of Counseling Psychology, 39(4), 447-452. doi: 10.1037/0022-0167.39.4.447

Matthieu, M., \& Ivanoff, A. (2006). Treatment of human-caused trauma: Attrition in the adult outcomes research. Journal of Interpersonal Violence, 21, 1654-1665. doi:10.1177 /0886260506294243

Mennicke, S. A., Lent, R. W., \& Burgoyne, K. L. (1988). Premature termination from university counseling. Journal of Counseling and Development, 66, 458-465. doi: 10.1002/j.1556 -6676.1988.tb00788.x

Merikangas, K. R., Nakamura, E. G., \& Kessler, R. C. (2010). Epidemiology of mental disorders in children and adolescence. Dialogues in Clinical Neuroscience, 11, 7-20.

Naar-King, S. (2011). Motivational interviewing in adolescent treatment. Canadian Journal of Psychiatry, 56(11), 651-656.

National Alliance on Mental Illness. (2013). Mental illness facts and numbers. Retrieved from http://www.nami.org/factsheets/mentalillness_factsheet.pdf

Pagnin, D. de Queiroz, V., \& Saggese, E. G. (2004). Predictors of attrition from day treatment of adolescents with substance-related disorders. Addictive Behaviors 30(2005), 1065-1069. doi:10.1016/j.addbeh.2004.09.013

Pallant, J. (2013). SPSS survival manual (5 ${ }^{\text {th }}$ ed.). Maidenhead, Berkshire, England: Open University Press, McGraw Hill.

Piaget, J. (1964). Part 1: Cognitive development in children: Piaget development and learning. Journal of Research in Science Teaching, 2(3), 176-186. doi:10.1002/tea.3660020306 
Poulsen, J., \& French, A. (2002). Discriminant function analysis (DA). [PDF document.] Retrieved from http://userwww.sfsu.edu/ efc/classes/biol710/discrim/discrim.pdf

Prinz, R., \& Milller, G. (1994). Family-based treatment for childhood antisocial behavior: Experimental influences on drop out and engagement. Journal of Consulting and Clinical Psychology, 62, 645-650. doi:10.1037/0022-006X.62.3.645

Reis, B. F., \& Brown, L. G. (1999). Reducing psychotherapy dropouts: maximizing perspective convergence in the psychotherapy dyad. Psychotheapy, 36(2), 123-136. doi: 10.1037 /h0087822

Rescorla, L., Achenbach, T.M., Ivanova, M. Y., Dumenci, L., Almqvist, F., Bilenberg, N., Bird, H., ... Verhulst, F. (2007). Epidemiological comparisons of problems and positive qualities reported by adolescents in 24 countries. Journal of Consulting and Clinical Psychology, 75, 351-358. doi:10.1037/0022 $-006 X .75 .2 .351$

Sheras, P. L., Abidin, R. R., \& Konold, T. R. (1998). Stress index for parents of adolescents. Odessa, FL: Psychological Assessment Resources.

Sherry, A. (2006). Discriminant analysis in counseling psychology research. Counseling Psychologist, 35, 661-683. doi:10.1177/0011000006287103

Siegel, A. W., Cousins, J. H., Rubovits, D. S., Parsons, J. T., Lavery, B., \& Crowley, C. L. (1994). Adolescents' perceptions of the benefits and risks of their own risk taking. Journal of Emotional and Behavioral Disorders, 2(2), 89-98. doi: 10.1177 $/ 106342669400200203$ 
Smetana, J. G., Metzger, A., Gettman, D. C., \& Campione-Barr, N. (2006). Disclosure and secrecy in adolescent-parent relationships. Child Development, 77(1), 201-217. doi: 10.1111/j.1467-8624.2006.00865.x

Snyder, C. M., \& Anderson, S. A. (2009). An examination of mandated versus voluntary referral as a determinant of clinical outcome. Journal of Marital and Family Therapy, 35(3), 278292. doi:10.111/j.1752-0606.2009.00118.x

Steinberg, L., \& Morris, A. (2001). Adolescent development. Annual Review of Psychology, 52, 83-110. doi:10.1146/annurev.psych.52.1.83

Sunseri, P. A. (2003). Predicting treatment termination due to running away among adolescents in residential care. Residential Treatment for Children \& Youth, 21(2), 43-60. doi: 10.1300/J007v21n02_03

Tabachnick, B. G., \& Fidell, L. S. (2001). Using multivariate statistics (4th ed.). Needham Heights, MA: Allyn \& Bacon.

Tan, J. O., \& Fegert, J. M. (2004). Capacity and competence and child and adolescent psychiatry. Health Care Analysis, 12(4), 285-294. doi:10.1007/s10728-004-6636-9

Thompson, B. (1992). Interpreting regression results: Beta weighs and structure coefficients are both important. Paper presented at the annual meeting of the American Educational Research Association, San Francisco, CA.

Tsai, M. H., \& Ray, D. C. (2011). Children in therapy: Learning from evaluation of universitybased community counseling clinical services. Children and Youth Services Review, 33, 901-909. doi:10.1016/j.childyouth.2010.12.011

U.S. Department of Health and Human Services. (2012). Youth risk behavior surveillance. Morbidity and Mortality Weekly Report 61(4). Retrieved from 
Venable, W. M., \& Thompson, B. (1998). Caretaker psychological factors predicting premature termination of children's counseling. Journal of Counseling and Development, 76, 286293. doi:10.1002/j.1556-6676.1998.tb02544.x

Webb, R. E., \& Widseth, J. C. (2005). Commentary on Gregory Hatchett’s “Reducing premature termination in university counseling centers”. Journal of College Student Psychotherapy, 19(4), 49-59. doi:10.1300/J035v19n04_04

Wentzel, K. R., \& Caldwell, K. (1997). Friendships, peer acceptance, and group membership: Relations to academic achievement in middle school. Child Development, 68(6), 11981209. doi:10.2307/1132301

Wierzbicki M., \& Pekarik, G. (1993). A meta-analysis of psychotherapy dropout. Professional Psychology: Research and Practice, 24(2), 190-195. doi:10.1037/0735-7028.24.2.190

World Health Organization. (2015). Adolescent health. Retrieved from http://www.who.int/topics/adolescent_health/en/

World Heath Organization. (2014). Prevention of mental disorders: Effective interventions and policy options. Geneva, Switzerland: World Health Organization. Retrieved from http://www.who.int/mental_health/evidence/en/prevention_of_mental_disorders_sr.pdf

Yap, M. B. H., Reavley, N., \& Jorn, A. F. (2012). Where would young people seek help for mental disorder and what stops them? Findings from an Australian national survey. Journal of Affective Disorders, 147(2013), 255-261. 